\title{
Second spectrum of Chromium (Cr II) Part I: New energy level reanalysis
}

\author{
Safa Bouazza ${ }^{\mathrm{a}, *}$, Pascal Quinet ${ }^{\mathrm{b}, \mathrm{c}}$, Patrick Palmeri ${ }^{\mathrm{b}}$ \\ a Département de Physique, Université de Reims-Champagne, UFR SEN, BP 1039 F-51687 Reims Cedex 2, France \\ b Physique Atomique et Astrophysique, Université de Mons UMONS, B-7000 Mons, Belgium \\ c IPNAS, Université de Liège, B-4000 Liège, Belgium
}

\section{A R T I C L E I N F O}

\section{Article history:}

Received 24 March 2017

Received in revised form 10 May 2017

Accepted 10 May 2017

Available online 7 June 2017

\section{Keywords:}

Energy-levels

Leading components

Landé $g_{j}$-factor

Chromium first ion

\begin{abstract}
A B S T R A C T
Using a linked-parametric technique of level-fitting calculations in a multiconfiguration basis, a parametric fine structure (fs) analysis for both even and odd parities of $\mathrm{Cr}$ II was achieved. For the first time the fs parameter, magnetic Lande $g$-factor and the percentage of leading eigenvector values for more than one thousand levels are determined and given for these two sets. This study has led us to confirm in the whole the well founded basis of NIST team works but inevitably also to exchange assignments of some levels classified in earlier lists of energy levels and to shift positions of some quartets like ${ }^{4} F_{J}$ for instance. Furthermore we take this opportunity to add our predicted doublet, quartet and sextet locations for missing experimental levels up to $124,000 \mathrm{~cm}^{-1}$.
\end{abstract}

(C) 2017 Elsevier Inc. All rights reserved.

\footnotetext{
* Corresponding author.

E-mail address: safa.bouazza@univ-reims.fr (S. Bouazza).
} 
Contents

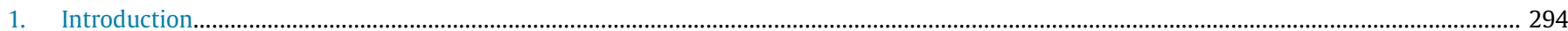

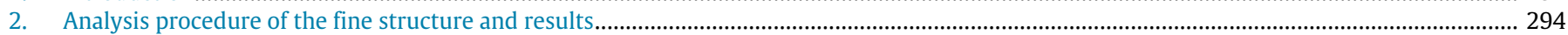

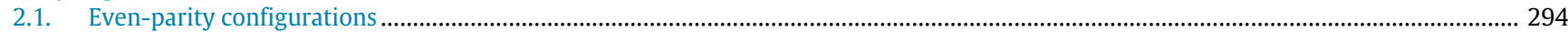

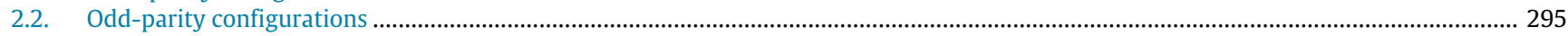

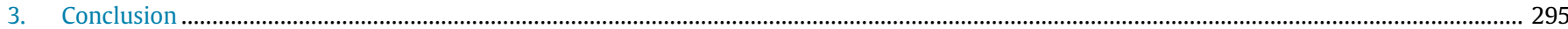

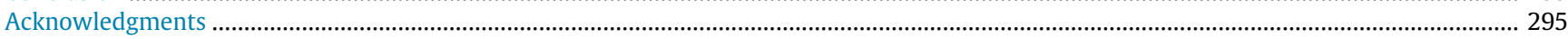

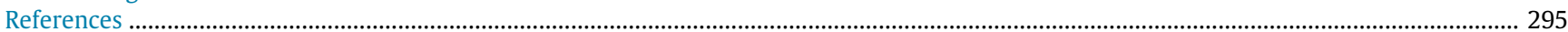

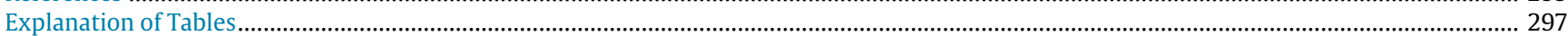

Table 1. Comparison of the experimental and calculated energy values, percentages of first and second components and $g_{J}$-factors for

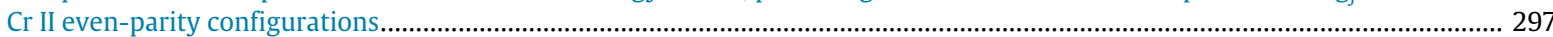

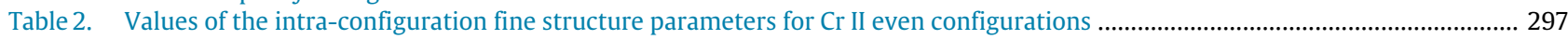

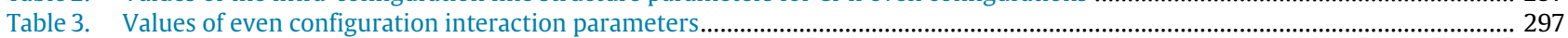

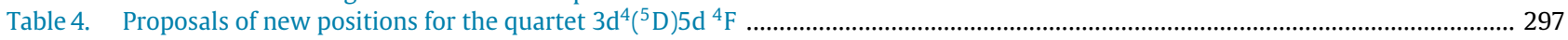

Table 5. Comparison of the experimental and calculated energy values, percentages of first and second components and $g_{j}$-factors for

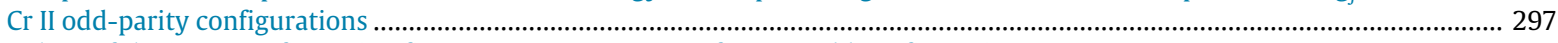

Table 6. Values of the intra-configuration fine structure parameters for $\mathrm{Cr}$ II odd configurations ............................................................. 297

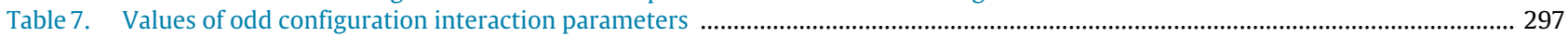

\section{Introduction}

Chromium is the first element in group 6, of the transition metals, well known for its remarkable magnetic properties: it is the only elemental solid which shows antiferromagnetic ordering at room temperature and below; above $38{ }^{\circ} \mathrm{C}$ it changes to paramagnetic. Naturally occurring chromium is composed of three stable isotopes: ${ }^{52} \mathrm{Cr},{ }^{53} \mathrm{Cr}$ and ${ }^{54} \mathrm{Cr}$, with abundances of $83.79 \%, 9.50 \%$ and $2.37 \%$ and nuclear spins of $0,3 / 2$ and 0 , respectively. Consequentially hyperfine structure (hfs) splitting of only enriched ${ }^{53} \mathrm{Cr}$ exists and was possible to evaluate like in [1,2] for atomic chromium but unfortunately no similar observations were achieved for ionic chromium. Furthermore, up to now, no isotope shift measurements are found in literature. As in many cases for different elements, Kiess [3] initiated the Cr II spectrum analysis. He reported observations of spectra excited in direct current arcs and condensed sparks between chromium electrodes. He succeeded in classifying of 1843 lines linking 138 even-parity levels of $3 d^{5}, 3 d^{4} 4 s$ and $3 d^{3} 4 s^{2}$ configurations with 139 oddparity levels belonging to only one configuration: $3 d^{4} 4 p$. In 1952 Moore published a compilation of the energy levels and some of its ions [4]. This work was updated by Sugar and Corliss in 1985 [5]. Johansson extended the Kiess study a half century later, particularly in the near-infrared region and analyzed 450 additional levels [6]. Sansonetti et al. [7,8] reported in turn new observations of $\mathrm{Cr}$ II some years later, in the near-ultra-violet region 1140-3400 A, using $10.7 \mathrm{~m}$ normal incidence vacuum spectrograph and FT700 vacuum ultraviolet Fourier transform spectrometer and then more than 3600 lines were classified linking 283 even levels and 368 odd levels. This work was extended two years later [8] by a large number of measurements in the region $2850-37,900 \AA$, presenting a list of more than 5300 lines linking this time 456 even and 457 odd levels. In this laborious compilation one can find highly excited level positions of the $3 d^{4} 5 g, 3 d^{4} 6 g$ and even $3 d^{4} 6 h$ configurations, up to $124,500 \mathrm{~cm}^{-1}$. Finally, let us point also the useful implication in this item of Saloman who gave a general survey of the situation [9].

The aim of this study is to perform oscillator strength parameterization and Hartree-Fock eigenvector calculations. In this regard, it is well known that the use of optimized eigenvectors obtained through a least-squares fitting of the calculated energy levels to the available experimental data may considerably improve the final results, not only for the atomic structure but also for radiative transition rates, magnetic Landé $g$-factors etc. Moreover, in many of our previous works, semi-empirically optimized eigenvectors turned out very useful for the confirmation of, or identification of errors in, tentative energy-level analyses. It also helped, on the one hand, in ascertaining the proper spectroscopic designation for identifying experimentally measured but not classified levels and, on the other hand, in predicting energy values of missing (not experimentally observed) levels. We want to take advantage of these excellent works of the NIST team to get level eigenvectors in order to transform into actual intermediate coupling the transition matrix beforehand obtained in pure LS coupling with help of Racah algebra [10-12]. In order to determine the radiative transition rates in the near future, we first need to deeply investigate both oddand even-parities, i.e. two sets of six and eleven configurations respectively in this work, some of whose of their energy levels are determined experimentally. As severe tests for level eigenvector validity one can use generally experimental hfs data. In the absence of the latter in the case of $\mathrm{Cr}$ II we will turn our efforts towards experimental oscillator strength or transition probability values, plentifully available in literature [13-16].

\section{Analysis procedure of the fine structure and results}

\subsection{Even-parity configurations}

We gave in our previous papers all details of the analysis procedure used to study fine structure of atoms and ions, see for instance [17-21]. Nevertheless we prefer to remind the reader that this procedure includes electrostatic and spin dependent interactions, which are represented by the Slater integrals $F^{k}, G^{k}, R^{k}$ and the spin-orbit parameters $\zeta_{n l}$. In our model, the configuration interaction was considered among the following even-parity configurations:

$$
3 d^{5}+\sum_{n=4}^{n=9} 3 d^{4} n s+\sum_{n^{\prime}=4}^{n^{\prime}=6} 3 d^{4} n^{\prime} d+3 d^{3} 4 s^{2}
$$

The interaction effects with distant configurations influencing the term structure were taken into account by two body parameters $\alpha$ and $\beta$ representing the one- and two-electron excitations respectively and by the three body parameters $T\left(\mathrm{~d}^{2} \mathrm{~s}\right), T 2(22)$ and T3(42). The effects of excitations of one electron from a closed shell to an open one were taken into account by electrostatically correlated spin-orbit interaction [22,23]. The other details of the whole procedure used here are described also in Refs. [24-26]. The fs least squares fitting procedure has been carried out over 
276 energy levels attributed to this extended model. With 445 parameters, 31 of which being treated as free, a very good fit has been achieved, resulting in a mean deviation for the energy level values of $\sigma(E)=57 \mathrm{~cm}^{-1}$. In Table 1 are given the observed energy levels, calculated eigenvalues, percentages of first and second components of the wave functions and their corresponding LS term designations. Moreover in this Table the calculated Landé $g_{J}$ factors, deduced from the eigenvector compositions, are compared with experimental ones when they are available in the literature. Tables 2 and 3 contain the values of fs parameters obtained through our fitting procedure. We have mentioned the number of experimental works in the Introduction as large even if many observed energy levels are missing. This is due mainly to the hard and long perusal and careful interpretation of recorded spectra obtained with the help of Fourier Transform spectrometer. For this reason we propose our predicted values in the column 3 of Table 2 to suggest the positions of missing energy levels absent in column 2 with rather quite acceptable accuracy. In this work we have discarded or shifted some questionable energy levels, particularly those concerning $3 d^{4} 5 d{ }^{4} F$. We suggest clarifying the situation of these quartets $3 d^{4} 5 d{ }^{4} F$ since from our analysis these levels should be located around proposed values given in Table 4 within uncertainty interval of about $50 \mathrm{~cm}^{-1}$. We have to point out also other divergences with literature data about $3 d^{4}\left({ }^{5} D\right) 5 d{ }^{6} S_{5 / 2}$ and $3 d^{4}\left({ }^{5} \mathrm{D}\right) 6 \mathrm{~d}{ }^{6} S_{5 / 2}$. We propose $111,469 \mathrm{~cm}^{-1}$ and $119,789 \mathrm{~cm}^{-1}$ respectively instead of $109394.430 \mathrm{~cm}^{-1}$ and $117672.453 \mathrm{~cm}^{-1}$. In Table 2 we also give our predicted values up to $124000 \mathrm{~cm}^{-1}$ in some cases, value close enough to ionization energy: 132971.02 $(0.12) \mathrm{cm}^{-1}$ [27]. In general one can observe a satisfactory agreement between the experimental values given in literature and our calculated data. In [27], Sansonetti et al. corrected some assignments proposed two years earlier [8] (some quartets belong rather to $3 d^{4} 4 d$ than to $3 d^{4} 5 d$ for instance) and we confirm these changes. Furthermore we agree totally with the optimized energy levels given in Table 3 of [27]. Nevertheless in the energy interval $103,000-108,000 \mathrm{~cm}^{-1}$ Sansonetti et al. [27] list the positions for $3 d^{4}\left({ }^{3} F\right) d^{4} F, 3 d^{4}\left({ }^{3} P\right) 4 d^{4} F$ and $3 d^{4}\left({ }^{3} H\right) 4 d^{4} F$ levels while Saloman [9] preferred to omit these level positions. We have found courageous that Sansonetti provided these questionable data since we think these levels belong really to $3 \mathrm{~d}^{4} 4 \mathrm{~d}$ but there remain sometimes some issues in the level labeling.

\subsection{Odd-parity configurations}

From our experience in fs studies we know that the investigations of odd-parity configuration levels in neutral and lowly ionized iron-group atoms are more difficult than those concerning even-parity configuration ones due to the presence of complex configuration-interaction effects particularly between configurations of the type $3 d^{k} 4 p, 3 d^{k-1} 4 s 4 p$ and $3 d^{k-2} 4 s^{2} 4 p$. In the case of the $\mathrm{Cr}$ II spectrum, the levels of $3 \mathrm{~d}^{3} 4 \mathrm{~s} 4 \mathrm{p}$ overlap levels from both the $3 d^{2} 4 s^{2} 4 p$ and $3 d^{4} 4 p$ configurations and generally this entanglement is hard to delimit. In this analysis we have considered only configurations whose experimental energy levels are given in literature, namely:

$3 d^{4} 4 p+3 d^{4} 5 p+3 d^{4} 6 p+3 d^{3} 4 s 4 p+3 d^{4} 4 f+3 d^{4} 5 f$

in order to avoid a kind of absorption of fs parameters in the presence of level resonance like electrostatic repulsions for instance. The quasi-totality of experimentally known odd-parity levels, located up to $124,000 \mathrm{~cm}^{-1}$, i.e. 354 levels, were fitted using 23 variable parameters among a total number of 245 . Since we have found some wrong experimental levels belonging to the evenparity configurations we first have paid attention to looking for questionable experimental odd-parity levels. For this purpose we have discarded at the beginning the level $3 \mathrm{~d}^{3}\left({ }^{4} \mathrm{~F}\right) 4 \mathrm{~s} 4 \mathrm{p}\left({ }^{1} \mathrm{P}\right){ }^{4} \mathrm{~F}_{9 / 2}$ at
$105203.460 \mathrm{~cm}^{-1}$ because its fitting was very bad. The levels of this quartet might be located higher. In [8] Sansonetti et al. have omitted to list 5 levels of the sextet $3 d^{4}\left({ }^{5} D\right) 5 p{ }^{6} F$, giving only the level ${ }^{6} \mathrm{~F}_{3 / 2}$. Yet these levels, given previously in [7] exist. In Table 5 we give our fitting procedure results with the calculated $g_{J}-$ value and leading eigenvector components and in Tables 6-7 are displayed the fs radial parameter values.

\section{Conclusion}

We took advantage of these huge and excellent works of the NIST team, giving a relevant number of experimental energy levels (more than 500) of the second spectrum of chromium to make a list of at least $1000 \mathrm{Cr}$ II energy levels with high reliability, predicting more than 500 of them, missing in previous works. It is a pity that a Zeeman study was not achieved in the same way because sometimes we have faced some problems and experimental Landéfactors would surely help us to take reasonable decisions, recurring to a comparison with our calculated $g_{J}$ values. Despite this deficiency we have performed successful fitting procedures with low standard deviations and then without these fingerprints we remain optimistic concerning our computed data and our change proposals too. We will confirm the contributions of this fruitful study in Part II, Part III and Part IV of this global work on the $\mathrm{Cr}$ II spectrum, showing very satisfactory agreements between experimental oscillator strengths and our calculated data obtained thanks to our deduced level eigenvector amplitudes, strengthening by the way the well founded basis of our fine structure analysis.

\section{Acknowledgments}

PQ and PP are respectively Research Director and Research Associate of the Belgian Fund for Scientific Research F.R.S.-FNRS. Financial support from this organization is deeply acknowledged. SB thanks deeply Dr. J.C. Boisson for technical assistance.

\section{References}

[1] T. Reinhardt, J. Maichel, M. Bauman, J. Krüger, Z. Phys. D 34 (1995) 87.

[2] J. Marek, Astron. Astrophys. 44 (1975) 69.

[3] C.C. Kiess, J. Res. Natl. Bur. Stand. 47 (1951) 387.

[4] C.E. Moore, Atomic Energy Levels, Vol. II, National Bureau of Standards, (US), Circular N 467, Washigton DC, 1952.

[5] J. Sugar, C. Corliss, J. Phys. Chem. Ref. Data 14 (Suppl. 2) (1985) 279.

[6] S. Johansson, T. Zethson, H. Hartman, et al., Astron. Astrophys. 361 (2000) 977.

[7] C.J. Sansonetti, F. Kerber, J. Reader, M.R. Rosa, Astrophys. J. Suppl. 153 (2004) 555.

[8] C.J. Sansonetti, G. Nave, J. Reader, F. Kerber, Astrophys. J. Suppl. Ser. 202 (2012) 15.

[9] E.B. Saloman, J. Phys. Chem. Ref. Data 41 (2012) N4.

[10] J. Ruckowski, M. Elantkowska, J. Dembczynski, J. Quant. Spectrosc. Radiat. Transfer 149 (2014) 168.

[11] J. Ruckowski, S. Bouazza, M. Elantkowska, J. Dembczynski, J. Quant. Spectrosc. Radiat. Transfer 155 (2015) 1.

[12] S. Bouazza, J. Spectrosc. Radiat. Transfer 187 (2017) 280.

[13] H. Nilsson, G. Lyung, H. Lundberg, K.E. Nielsen, Astron. Astrophys. 445 (2006) 1165.

[14] J. Gurell, H. Nilsson, L. Engström, H. Lundberg, R. Blackwell-Whitehead, K.E. Nielsen, S. Mannervik, Astron. Astrophys. 511 (2010) A68.

[15] L. Engström, H. Lundberg, H. Nilsson, H. Hartman, E. Bäckström, Astron. Astrophys. 570 (2014) A34.

[16] S.D. Bergeson, J.E. Lawler, Astrophs. J. 408 (1993) (1993) 382.

[17] J. Dembczynski E. Stachowska, G. Szawiola, J. Ruckowski, Eur. Phys. J. D 4 (1998) 39.

[18] S. Bouazza, Can. J. Phys. 94 (2016) (2016) 1310.

[19] J. Dembczynski, S. Bouazza, G. Szawiola, J. Ruckowski, J. Physique 7 (1997) 1175.

[20] S. Bouazza, P. Quinet, P. Palmeri, J. Quant. Spectrosc. Radiat. Transfer 163 (2015) 39.

[21] P. Quinet, S. Bouazza, P. Palmeri, J. Quant. Spectrosc. Radiat. Transfer 164 (2015) 193.

[22] J. Dembczynski, M. Elantkowska, B. Furmann, J. Ruckowski, J. Phys. B. 43 (2010) 6. 
[23] J. Dembczynski, M. Elantkowska, J. Ruckowski, D. Stefanska, At. Data Nucl. Data Tables 93 (2007) 1

[24] J. Dembczynski, G. Szawiola, M. Elantkowska, E. Stachowska, J. Ruckowski, Phys. Scr. 54 (1996) 5.

[25] E. Stachowska, J. Dembczynski, L. Windholz, J. Ruckowski, M. Elantkowska, At. Data Nucl. Data Tables 113 (2017) 350
[26] M. Elantkowska, J. Ruckowski, J. Dembczynski, Eur. Phys. J. Plus 130 (2015) 1

[27] C.J. Sansonetti, G. Nave, Astrophys. J. Suppl. Ser. 213 (2014) 28.

[28] R.D. Cowan, The Theory of Atomic Structure Spectra, University of California Press, Berkeley, CA, 1981. 


\section{Explanation of Tables}

Table 1.

Table 2. Configuration: Parameter: Value:

Table 3.

Table 4

Table 5

Comparison of the experimental and calculated energy values, percentages of first and second components and $g_{J}$-factors for $\mathrm{Cr}$ II even-parity configurations

$J$-value:

$E_{o b s}$ :

$E_{\text {calc }}$ :

Largest eigenvalue component:

Total angular momentum of considered level.

Observed energy level value in $\mathrm{cm}^{-1}$.

Calculated eigenvalue in $\mathrm{cm}^{-1}$.

Percentage contribution of the main eigenvector component. In ${ }^{2 S^{\prime}+1} L^{\prime} ;{ }^{2 S+1} L,{ }^{2 S^{\prime}+1} L^{\prime}$ stands for the parent term.

Next largest component: Percentage contribution of the second eigenvector component. In ${ }^{2 S^{\prime}+1} L^{\prime} ; \quad{ }^{2 S+1} L,{ }^{2 S^{\prime}+1} L^{\prime}$ stands for the parent term.

Calc. $g_{J}$ :

Obs. $g_{J}$ :

Calculated Landé $g_{J}$-factor value using eigenvector level composition. Measured Landé $g_{J}$-factor value by means of Zeeman structure.

Values of the intra-configuration fine structure parameters for $\mathrm{Cr}$ II even configurations

Name ofthe considered configuration in this study.

Name of radial parameter.

Radial parameter value in $\mathrm{cm}^{-1}$. Standard deviation is given in

parentheses.

${ }^{f}$ Frozen to zero. ${ }^{d}$ Deduced from $a$ b initio or fs calculations.

Values of even configuration interaction parameters

Configurations:

Parameter:

Value:

Designations of interacting configurations.

Name of radial parameter whose meanings are given in [26].

Radial parameter value in $\mathrm{cm}^{-1}$. Standard deviation is given in parentheses.

Proposals of new positions for the quartet $3 d^{4}\left({ }^{5} D\right) 5 d{ }^{4} F$

J-value:

Position given in [27]:

Total angular momentum of considered level.

Experimental energy level in $\mathrm{cm}^{-1}$ given in Ref. [27].

Our proposed position:

Obtained energy level in $\mathrm{cm}^{-1}$ by means of our calculations.

Comparison of the experimental and calculated energy values, percentages of first and second components and $g_{J}$-factors

for Cr II odd-parity configurations

$J$-value:

$E_{\text {obs }}$ :

$E_{\text {calc }}$ :

Largest eigenvalue component:

Total angular momentum of considered level.

Observed energy level value in $\mathrm{cm}^{-1}$.

Calculated eigenvalue in $\mathrm{cm}^{-1}$.

Percentage contribution of the main eigenvector component.

In ${ }^{2 S^{\prime}+1} L^{\prime} ;{ }^{2 S+1} L,{ }^{2 S^{\prime}+1} L^{\prime}$ stands for the parent term.

In $3 \mathrm{~d}^{3} 4 \mathrm{~s} 4 \mathrm{p}^{2 S^{\prime \prime}+1} L^{\prime \prime} .{ }^{2 S^{\prime}+1} L^{\prime} ;{ }^{2 S+1} L,{ }^{2 S^{\prime}+1} L^{\prime}$ and ${ }^{2 S^{\prime \prime}+1} L^{\prime \prime}$ stand

Next largest component:

respectively for the parent and grandparent terms.

Percentage contribution of the second eigenvector component.

In ${ }^{2 S^{\prime}+1} L^{\prime} ;{ }^{2 S+1} L,{ }^{2 S^{\prime}+1} L^{\prime}$ stands for the parent term.

In $3 \mathrm{~d}^{3} 4 \mathrm{~s} 4 \mathrm{p}^{2 S^{\prime \prime}+1} L^{\prime \prime} .2 S^{\prime}+1 L^{\prime} ;{ }^{2 S+1} L,{ }^{2 S^{\prime}+1} L^{\prime}$ and $2 S^{\prime \prime}+1 L^{\prime \prime}$ stand

respectively for the parent and grandparent terms.

Calculated Landé $g_{J}$-factor value using eigenvector level composition.

:alc. g]:

Measured Landé $g_{J}$-factor value by means of Zeeman structure.

Table 6

Values of the intra-configuration fine structure parameters for $\mathrm{Cr}$ II odd configurations

Configuration:

Parameter:

Value:

Name of the considered configuration in this study.

Name of radial parameter.

Radial parameter value in $\mathrm{cm}^{-1}$. Standard deviation is given in parentheses.

${ }^{f}$ Frozen to zero. ${ }^{d}$ Deduced from $a$ b initio or fs calculations.

Table 7

Values of odd configuration interaction parameters

Configurations:

Parameter:

Designations of interacting configurations.

Name of radial parameter whose meanings are given in [25].

Radial parameter value in $\mathrm{cm}^{-1}$. Standard deviation is given in parentheses.

${ }^{d}$ Deduced from $a b$ initio or fs calculations. 
Table 1

Comparison of the experimental and calculated energy values, percentages of first and second components and $g_{J}$-factors for Cr II even-parity configurations.

\begin{tabular}{|c|c|c|c|c|c|c|c|c|}
\hline \multirow{2}{*}{$\begin{array}{c}J \text {-value } \\
0.5\end{array}$} & \multirow{2}{*}{$\begin{array}{c}E_{o b s} \\
\left(\mathrm{~cm}^{-1}\right) \\
{[8,27]}\end{array}$} & \multirow[t]{2}{*}{$\begin{array}{c}E_{\text {calc }} \\
\left(\mathrm{cm}^{-1}\right)\end{array}$} & \multicolumn{2}{|c|}{$\begin{array}{l}\text { Largest eigenvalue component } \\
\qquad(\%)\end{array}$} & \multicolumn{2}{|c|}{$\begin{array}{l}\text { Next largest eigenvalue component } \\
\text { (\%) }\end{array}$} & \multirow[t]{2}{*}{ Calc. $g_{J}$} & \multirow{2}{*}{$\begin{array}{c}\text { Obs. } g_{J} \\
{[5]}\end{array}$} \\
\hline & & & & & & & & \\
\hline & 11961.747 & 11908 & 99.84 & $3 d^{4} 4 s^{5} D ;{ }^{6} D$ & 0.05 & $3 d^{4} 4 s^{3} \mathrm{P} ;{ }^{4} \mathrm{P}$ & 3.338 & 3.323 \\
\hline & 19528.230 & 19510 & 95.21 & $3 d^{4} 4 s^{5} D ;{ }^{4} D$ & 4.34 & $3 d^{54} D$ & -0.001 & 0.000 \\
\hline & 21823.725 & 21873 & 97.05 & $3 d^{54} \mathrm{P}$ & 1.00 & $3 \mathrm{~d}^{4} 4 \mathrm{~s}^{3} \mathrm{P} ;{ }^{4} \mathrm{P}$ & 2.664 & 2.693 \\
\hline & 25035.346 & 25062 & 94.12 & $3 d^{54} D$ & 4.41 & $3 d^{4} 4 s^{5} D ;{ }^{4} D$ & 0.003 & 0.045 \\
\hline & 29951.818 & 29959 & 57.86 & $3 d^{4} 4 s^{3} \mathrm{P} ;{ }^{4} \mathrm{P}$ & 40.32 & $3 d^{4} 4 s^{3} \mathrm{P} ;{ }^{4} \mathrm{P}$ & 2.666 & 2.685 \\
\hline & 34659.242 & 34674 & 58.61 & $3 d^{4} 4 s^{3} P ;{ }^{2} P$ & 40.45 & $3 d^{4} 4 s^{3} \mathrm{P} ;{ }^{2} \mathrm{P}$ & 0.671 & 0.67 \\
\hline & 38396.188 & 38409 & 99.21 & $3 d^{4} 4 s^{3} D ;{ }^{4} D$ & 0.46 & $3 d^{54} D$ & 0.000 & \\
\hline & 40415.090 & 40364 & 53.58 & $3 d^{4} 4 s{ }^{1} s ;{ }^{2} s$ & 31.31 & $3 d^{52} S$ & 1.999 & \\
\hline & 44307.078 & 44287 & 67.38 & $3 d^{52} S$ & 24.22 & $3 d^{4} 4 s{ }^{1} s ;{ }^{2} S$ & 2.002 & \\
\hline & 55626.102 & 55655 & 58.96 & $3 d^{4} 4 s^{3} \mathrm{P} ;{ }^{4} \mathrm{P}$ & 40.54 & $3 d^{4} 4 s^{3} \mathrm{P} ;{ }^{4} \mathrm{P}$ & 2.669 & \\
\hline & 59526.684 & 59491 & 47.13 & $3 \mathrm{~d}^{4} 4 \mathrm{~s}^{3} \mathrm{P} ;{ }^{2} \mathrm{P}$ & 34.02 & $3 d^{4} 4 s^{3} \mathrm{P} ;{ }^{2} \mathrm{P}$ & 0.667 & \\
\hline & & 63372 & 64.01 & $3 d^{52} \mathrm{P}$ & 15.56 & $3 d^{3} 4 s^{22} P$ & 0.671 & \\
\hline & 65882.438 & 65895 & 98.88 & $3 d^{3} 4 s^{24} P$ & 0.43 & $3 d^{54} P$ & 2.659 & \\
\hline & & 70925 & 83.11 & $3 d^{3} 4 s^{22} P$ & 15.5 & $3 d^{52} P$ & 0.673 & \\
\hline & 82692.008 & 82677 & 99.63 & $3 d^{4} 5 s^{5} D ;{ }^{6} D$ & 0.09 & $3 d^{4} 4 d^{5} D ;{ }^{6} D$ & 3.337 & \\
\hline & 84209.781 & 84218 & 99.72 & $3 d^{4} 5 s^{5} D ;{ }^{4} D$ & 0.09 & $3 d^{4} 4 s^{5} D ;{ }^{4} D$ & 0.000 & \\
\hline & 87453.484 & 87368 & 95.41 & $3 d^{4} 4 d^{5} D ;{ }^{6} D$ & 3.88 & $3 d^{4} 4 d^{5} D ;{ }^{6} F$ & 3.180 & \\
\hline & 87594.562 & 87615 & 95.5 & $3 d^{4} 4 d^{5} D ; 6 F$ & 3.89 & $3 d^{4} 4 d^{5} D ;{ }^{6} D$ & -0.512 & \\
\hline & 89254.469 & 89246 & 89.28 & $3 d^{4} 4 d^{5} D ;{ }^{4} P$ & 7.14 & $3 d^{4} 4 d^{5} D ;{ }^{4} D$ & 2.474 & \\
\hline & 89651.617 & 89559 & 89.27 & $3 d^{4} 4 d^{5} D ;{ }^{4} D$ & 7.14 & $3 d^{4} 4 d^{5} D ;{ }^{4} P$ & 0.195 & \\
\hline & & 94771 & 77.64 & $3 d^{4} 4 s{ }^{1} s ;{ }^{2} S$ & 21.51 & $3 d^{4} 4 s^{1} s ;{ }^{2} S$ & 2.002 & \\
\hline & 99677.850 & 99706 & 59.62 & $3 d^{4} 5 s^{3} \mathrm{P} ;{ }^{4} \mathrm{P}$ & 37.31 & $3 \mathrm{~d}^{4} 5 \mathrm{~s}^{3} \mathrm{P} ;{ }^{4} \mathrm{P}$ & 2.617 & \\
\hline & 100782.820 & 100809 & 59.92 & $3 d^{4} 5 s^{3} P ;{ }^{2} P$ & 37.06 & $3 d^{4} 5 s^{3} \mathrm{P} ;{ }^{2} \mathrm{P}$ & 0.719 & \\
\hline & 105098.859 & 105135 & 99.7 & $3 d^{4} 6 s^{5} D ;{ }^{6} D$ & 0.13 & $3 d^{4} 6 s^{5} D ;{ }^{4} D$ & 3.334 & \\
\hline & & 105797 & 56.83 & $3 d^{4} 4 d^{3} F ;{ }^{4} D$ & 11.94 & $3 d^{4} 6 s^{5} D ;{ }^{4} D$ & 0.018 & \\
\hline & 105923.445 & 105862 & 87.2 & $3 d^{4} 6 s^{5} D ;{ }^{4} D$ & 6.41 & $3 d^{4} 4 d^{3} F ;{ }^{4} D$ & 0.006 & \\
\hline & $\cdot$ & & & & & & & \\
\hline & 115234.461 & 115235 & 98.73 & $3 d^{4} 7 s^{5} D ;{ }^{6} D$ & 1.04 & $3 d^{4} 7 s^{5} D ;{ }^{4} D$ & 3.303 & \\
\hline & & 115527 & 98.77 & $3 d^{4} 7 s^{5} D ;{ }^{4} D$ & 1.04 & $3 d^{4} 7 s^{5} D ;{ }^{6} D$ & 0.033 & \\
\hline & 116361.016 & 116348 & 77.29 & $3 d^{4} 6 d^{5} D ;{ }^{6} F$ & 21.6 & $3 d^{4} 6 d^{5} D ;{ }^{6} D$ & 0.208 & \\
\hline & $\cdot$ & & & & & & & \\
\hline & 120702.898 & 120699 & 96.61 & $3 d^{4} 8 s^{5} D ;{ }^{6} D$ & 3.15 & $3 d^{4} 8 s^{5} \mathrm{D} ;{ }^{4} \mathrm{D}$ & 3.233 & \\
\hline \multicolumn{9}{|c|}{$2-5=-1$} \\
\hline & 12032.545 & 11980 & 99.86 & $3 d^{4} 4 s^{5} D ;{ }^{6} D$ & 0.03 & $3 d^{4} 4 s^{3} \mathrm{P} ;{ }^{4} \mathrm{P}$ & 1.869 & 1.867 \\
\hline & 19631.205 & 19614 & 94.97 & $3 d^{4} 4 s^{5} D ;{ }^{4} D$ & 4.54 & $3 d^{54} D$ & 1.201 & 1.192 \\
\hline & 21824.141 & 21865 & 96.65 & $3 d^{54} \mathrm{P}$ & 0.92 & $3 d^{4} 4 s^{3} \mathrm{P} ;{ }^{4} \mathrm{P}$ & 1.731 & 1.717 \\
\hline & 25042.760 & 25074 & 93.5 & $3 d^{54} D$ & 4.52 & $3 d^{4} 4 s^{5} D ;{ }^{4} D$ & 1.204 & 1.207 \\
\hline & 30307.365 & 30311 & 58.16 & $3 d^{4} 4 s^{3} \mathrm{P} ;{ }^{4} \mathrm{P}$ & 40.22 & $3 \mathrm{~d}^{4} 4 \mathrm{~s}^{3} \mathrm{P} ;{ }^{4} \mathrm{P}$ & 1.734 & 1.756 \\
\hline & 31082.885 & 31134 & 65.91 & $3 d^{4} 4 s^{3} F ;{ }^{4} F$ & 19.35 & $3 d^{4} 4 s^{3} F ;{ }^{4} F$ & 0.414 & 0.416 \\
\hline & 31531.172 & 31496 & 67.93 & $3 d^{52} D$ & 23.09 & $3 d^{52} D$ & 0.776 & \\
\hline & 32844.703 & 33029 & 84.75 & $3 d^{54} F$ & 6.86 & $3 d^{4} 4 s^{3} F ;{ }^{4} F$ & 0.408 & \\
\hline & 35355.898 & 35374 & 59.03 & $3 \mathrm{~d}^{4} 4 \mathrm{~s}^{3} \mathrm{P} ;{ }^{2} \mathrm{P}$ & 40.25 & $3 \mathrm{~d}^{4} 4 \mathrm{~s}^{3} \mathrm{P} ;{ }^{2} \mathrm{P}$ & 1.334 & 1.331 \\
\hline & 38362.383 & 38383 & 99.09 & $3 d^{4} 4 s^{3} D ;{ }^{4} D$ & 0.47 & $3 d^{54} D$ & 1.201 & \\
\hline & 42986.566 & 43026 & 92.93 & $3 d^{4} 4 s^{3} D ;{ }^{2} D$ & 4.32 & $3 d^{52} D$ & 0.800 & \\
\hline & 45669.398 & 45634 & 64.69 & $3 d^{4} 4 s^{1} D ;{ }^{2} D$ & 17.43 & $3 d^{4} 4 s^{1} D ;{ }^{2} D$ & 0.800 & \\
\hline & 47372.430 & 47317 & 75.78 & $3 d^{52} D$ & 12.38 & $3 d^{4} 4 s^{1} D ;{ }^{2} D$ & 0.800 & \\
\hline & 53051.270 & 53055 & 97.64 & $3 d^{3} 4 s^{24} F$ & 2.09 & $3 d^{54} F$ & 0.399 & \\
\hline & 54868.508 & 54894 & 76.42 & $3 d^{4} 4 s^{3} F ;{ }^{4} F$ & 23.02 & $3 d^{4} 4 s^{3} F ;{ }^{4} F$ & 0.399 & \\
\hline & 55398.672 & 55424 & 59 & $3 d^{4} 4 s^{3} \mathrm{P} ;{ }^{4} \mathrm{P}$ & 40.4 & $3 d^{4} 4 s^{3} \mathrm{P} ;{ }^{4} \mathrm{P}$ & 1.734 & \\
\hline & 59130.340 & 59117 & 49.13 & $3 d^{4} 4 s^{3} \mathrm{P} ;{ }^{2} \mathrm{P}$ & 34.96 & $3 d^{4} 4 s^{3} \mathrm{P} ;{ }^{2} \mathrm{P}$ & 1.335 & \\
\hline & & 63311 & 66.68 & $3 d^{52} \mathrm{P}$ & 15.1 & $3 d^{3} 4 s^{22} P$ & 1.334 & \\
\hline & 66010.273 & 66001 & 97.86 & $3 d^{3} 4 s^{24} P$ & 1.03 & $3 d^{52} P$ & 1.728 & \\
\hline & $\cdot$ & & & & & & & \\
\hline & 82763.664 & 82749 & 99.57 & $3 d^{4} 5 s^{5} D ;{ }^{6} D$ & 0.12 & $3 d^{4} 5 s^{5} D ;{ }^{4} D$ & 1.868 & \\
\hline & 84320.141 & 84329 & 99.67 & $3 d^{4} 5 s^{5} D ;{ }^{4} D$ & 0.13 & $3 d^{4} 5 s^{5} D ;{ }^{6} D$ & 1.201 & \\
\hline & 86165.266 & 86097 & 95.35 & $3 d^{4} 4 d^{5} D ;{ }^{4} S$ & 3.49 & $3 d^{4} 4 d^{5} D ;{ }^{6} P$ & 2.015 & \\
\hline & 86594.375 & 86527 & 99.02 & $3 d^{4} 4 d^{5} D ;{ }^{6} G$ & 0.44 & $3 d^{4} 5 d^{5} D ;{ }^{6} G$ & 0.002 & \\
\hline & 86667.719 & 86683 & 94.39 & $3 d^{4} 4 d^{5} D ;{ }^{6} P$ & 3.51 & $3 d^{4} 4 d^{5} D ;{ }^{4} S$ & 2.381 & \\
\hline & 87470.539 & 87397 & 86.55 & $3 d^{4} 4 d^{5} D ;{ }^{6} D$ & 11.37 & $3 d^{4} 4 d^{5} D ;{ }^{6} F$ & 1.783 & \\
\hline & 87666.203 & 87681 & 87.76 & $3 d^{4} 4 d^{5} D ;{ }^{6} F$ & 11.34 & $3 d^{4} 4 d^{5} D ;{ }^{6} D$ & 1.157 & \\
\hline & 89277.922 & 89264 & 79.68 & $3 d^{4} 4 d^{5} D ;{ }^{4} P$ & 16.3 & $3 d^{4} 4 d^{5} D ;{ }^{4} D$ & 1.645 & \\
\hline & 89724.172 & 89648 & 79.19 & $3 d^{4} 4 d^{5} D ;{ }^{4} D$ & 16.3 & $3 d^{4} 4 d^{5} D ;{ }^{4} P$ & 1.283 & \\
\hline & 90512.516 & 90566 & 94.39 & $3 d^{4} 4 d^{5} D ;{ }^{4} F$ & 1.23 & $3 d^{4} 4 d^{3} \mathrm{H} ;{ }^{4} \mathrm{~F}$ & 0.407 & \\
\hline & & 99957 & 75.4 & $3 d^{3} 4 s^{22} D$ & 22.79 & $3 d^{4} 4 s^{1} S ;{ }^{2} D$ & 0.800 & \\
\hline & 100040.172 & 100052 & 59.72 & $3 d^{4} 5 s^{3} \mathrm{P} ;{ }^{4} \mathrm{P}$ & 37 & $3 d^{4} 5 s^{3} P ;{ }^{4} \mathrm{P}$ & 1.722 & \\
\hline & 101245.000 & 101207 & 79.67 & $3 d^{4} 5 s^{3} F ;{ }^{4} F$ & 20.08 & $3 d^{4} 5 s^{3} F ;{ }^{4} F$ & 0.399 & \\
\hline
\end{tabular}


Table 1 (continued)

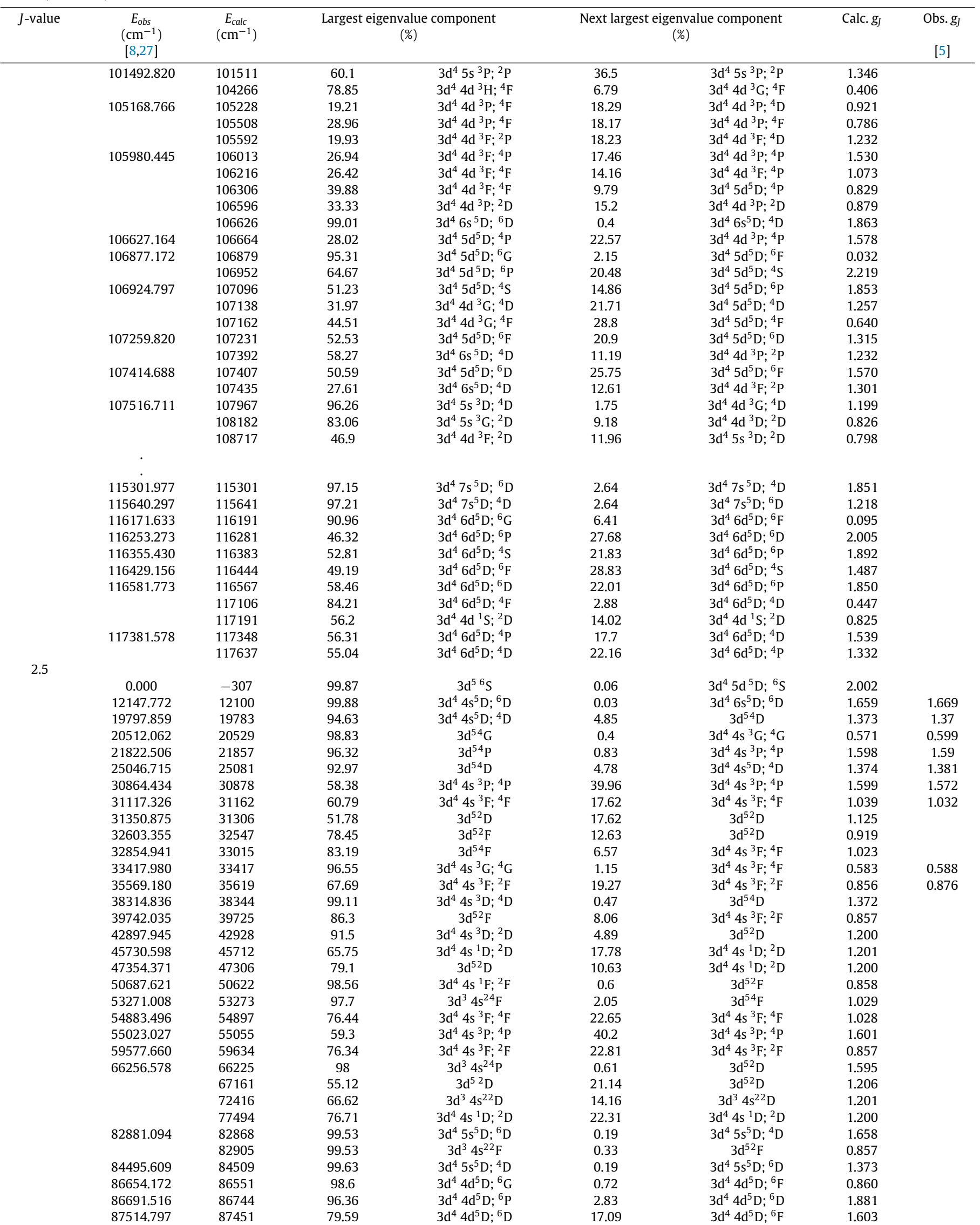


Table 1 (continued)

\begin{tabular}{|c|c|c|c|c|c|c|c|c|}
\hline$J$-value & \multirow{2}{*}{$\begin{array}{c}E_{o b s} \\
\left(\mathrm{~cm}^{-1}\right) \\
{[8,27]} \\
87759.062\end{array}$} & \multirow{2}{*}{$\begin{array}{c}E_{\text {calc }} \\
\left(\mathrm{cm}^{-1}\right)\end{array}$} & \multicolumn{2}{|c|}{$\begin{array}{l}\text { Largest eigenvalue component } \\
(\%)\end{array}$} & \multicolumn{2}{|c|}{$\begin{array}{c}\text { Next largest eigenvalue component } \\
(\%)\end{array}$} & Calc. $g_{J}$ & $\begin{array}{c}\text { Obs. } g_{J} \\
{[5]}\end{array}$ \\
\hline & & & 81.67 & $3 d^{4} 4 d^{5} D ;{ }^{6} F$ & 17.06 & $3 d^{4} 4 d^{5} D ;{ }^{6} D$ & 1.373 & \\
\hline & 89055.945 & 89169 & 96.39 & $3 d^{4} 4 d^{5} D ;{ }^{4} G$ & 1.22 & $3 \mathrm{~d}^{4} 4 \mathrm{~d}^{3} \mathrm{H} ;{ }^{4} \mathrm{G}$ & 0.573 & \\
\hline & 89336.812 & 89326 & 79.19 & $3 d^{4} 4 d^{5} D ;{ }^{4} P$ & 17.19 & $3 d^{4} 4 d^{5} D ;{ }^{4} D$ & 1.559 & \\
\hline & 89812.398 & 89746 & 77.45 & $3 d^{4} 4 d^{5} D ;{ }^{4} D$ & 17.34 & $3 d^{4} 4 d^{5} D ;{ }^{4} P$ & 1.407 & \\
\hline & 90608.961 & 90670 & 93.23 & $3 d^{4} 4 d^{5} D ;{ }^{4} F$ & 1.93 & $3 d^{4} 4 d^{5} D ;{ }^{4} D$ & 1.034 & \\
\hline & \multirow[t]{2}{*}{91955.336} & 91949 & 91.84 & $3 d^{4} 4 d^{5} D ;{ }^{6} S$ & 6.41 & $3 d^{4} 5 d^{5} D ;{ }^{6} S$ & 2.002 & \\
\hline & & 99783 & 76.65 & $3 d^{3} 4 s^{22} D$ & 21.47 & $3 d^{3} 4 s^{22} D$ & 1.200 & \\
\hline & 100650.461 & 100659 & 61.93 & $3 d^{4} 5 s^{3} P ;{ }^{4} P$ & 37.79 & $3 d^{4} 6 s^{3} \mathrm{P} ;{ }^{4} \mathrm{P}$ & 1.601 & \\
\hline & 101276.547 & 101235 & 79.23 & $3 d^{4} 5 s{ }^{3} F ;{ }^{4} F$ & 19.72 & $3 d^{4} 6 s^{3} F ;{ }^{4} F$ & 1.025 & \\
\hline & \multirow{5}{*}{102148.703} & 102139 & 78.25 & $3 d^{4} 5 s^{3} F ;{ }^{2} F$ & 19.47 & $3 d^{4} 6 s^{3} F ;{ }^{2} F$ & 0.852 & \\
\hline & & 103444 & 96.84 & $3 d^{4} 5 s^{3} G ;{ }^{4} G$ & 1.58 & $3 d^{4} 6 s^{3} F ;{ }^{2} F$ & 0.580 & \\
\hline & & 104299 & 78.29 & $3 d^{4} 4 d^{3} \mathrm{H} ;{ }^{4} \mathrm{~F}$ & 7.64 & $3 d^{4} 4 d^{3} G ;{ }^{4} F$ & 1.030 & \\
\hline & & 104893 & 74.29 & $3 d^{4} 4 d^{3} H ;{ }^{4} G$ & 12.45 & $3 d^{4} 5 d^{5} D ;{ }^{4} G$ & 0.588 & \\
\hline & & 105108 & 65.4 & $3 d^{4} 4 d^{3} \mathrm{H} ;{ }^{2} \mathrm{~F}$ & 10.44 & $3 d^{4} 4 d{ }^{3} \mathrm{P} ;{ }^{2} \mathrm{~F}$ & 0.901 & \\
\hline & \multirow[t]{2}{*}{105285.312} & 105358 & 21.34 & $3 d^{4} 4 d^{3} P ;{ }^{4} D$ & 17.84 & $3 d^{4} 4 d^{3} F ;{ }^{4} D$ & 1.231 & \\
\hline & & 105690 & 30.06 & $3 d^{4} 4 d^{3} \mathrm{P} ;{ }^{4} \mathrm{~F}$ & 18.89 & $3 d^{4} 4 d^{3} P ;{ }^{4} F$ & 1.153 & \\
\hline & 105923.438 & 105897 & 24.22 & $3 d^{4} 4 d^{3} \mathrm{P} ;{ }^{4} \mathrm{P}$ & 20.19 & $3 d^{4} 4 d{ }^{3} F ;{ }^{4} P$ & 1.508 & \\
\hline & 106095.602 & 106065 & 63.97 & $3 d^{4} 4 d^{3} F ;{ }^{4} G$ & 14.46 & $3 d^{4} 5 d^{5} D ;{ }^{4} G$ & 0.576 & \\
\hline & & 106244 & 38.39 & $3 d^{4} 4 d^{3} F ;{ }^{4} F$ & 9.41 & $3 d^{4} 4 d^{3} F ;{ }^{4} P$ & 1.214 & \\
\hline & & 106388 & 22.07 & $3 d^{4} 4 d^{3} F ;{ }^{4} F$ & 19.43 & $3 d^{4} 5 d^{5} D ;{ }^{4} P$ & 1.316 & \\
\hline & 106722.555 & 106649 & 15.01 & $3 d^{4} 5 d^{5} D ;{ }^{4} P$ & 13.36 & $3 d^{4} 4 d^{3} \mathrm{P} ;{ }^{4} \mathrm{D}$ & 1.351 & \\
\hline & & 106726 & 98.22 & $3 d^{4} 5 d^{5} D ;{ }^{6} D$ & 0.55 & $3 d^{4} 6 s^{5} D ;{ }^{4} D$ & 1.652 & \\
\hline & & 106741 & 26.58 & $3 d^{4} 4 d^{3} P ;{ }^{2} D$ & 12.75 & $3 d^{4} 4 d^{3} F ;{ }^{2} D$ & 1.304 & \\
\hline & 106929.375 & 106929 & 86.28 & $3 d^{4} 5 d^{5} D ;{ }^{6} G$ & 5.45 & $3 d^{4} 5 d^{5} D ;{ }^{6} F$ & 0.905 & \\
\hline & & 106970 & 51.58 & $3 d^{4} 4 d^{3} F ;{ }^{2} F$ & 10.97 & $3 d^{4} 4 d^{3} F ;{ }^{2} F$ & 0.851 & \\
\hline & 107025.250 & 107002 & 73.12 & $3 d^{4} 5 d^{5} D ; 6 P$ & 18.77 & $3 d^{4} 5 d^{5} D ;{ }^{6} D$ & 1.789 & \\
\hline & & 107233 & 43.92 & $3 d^{4} 4 d^{3} G ;{ }^{4} D$ & 31.85 & $3 d^{4} 5 d^{5} D ;{ }^{4} D$ & 1.370 & \\
\hline & & 107254 & 38.15 & $3 d^{4} 5 d^{5} D ;{ }^{6} F$ & 20.05 & $3 d^{4} 4 d^{3} G ;{ }^{4} F$ & 1.268 & \\
\hline & 107309.328 & 107291 & 47.23 & $3 d^{4} 4 d^{3} G ;{ }^{4} G$ & 27.69 & $3 d^{4} 5 d^{5} D ;{ }^{4} G$ & 0.663 & \\
\hline & & 107321 & 30.79 & $3 d^{4} 4 d^{3} G ;{ }^{4} F$ & 20.09 & $3 d^{4} 5 d^{5} D ;{ }^{4} F$ & 1.199 & \\
\hline & 107519.422 & 107521 & 56.94 & $3 d^{4} 5 d^{5} D ;{ }^{6} D$ & 31.07 & $3 d^{4} 5 d^{5} D ;{ }^{6} F$ & 1.554 & \\
\hline & 107597.609 & 107556 & 93.54 & $3 d^{4} 6 s^{5} D ;{ }^{4} D$ & 2.38 & $3 d^{4} 4 d^{3} G ;{ }^{4} D$ & 1.376 & \\
\hline & & 107888 & 35.14 & $3 d^{4} 4 d^{3} G ;{ }^{2} F$ & 21.56 & $3 d^{4} 4 d^{3} \mathrm{P} ;{ }^{2} \mathrm{~F}$ & 0.885 & \\
\hline & 107726.773 & 107931 & 92.97 & $3 d^{4} 5 s^{3} D ;{ }^{4} D$ & 2.25 & $3 d^{4} 4 d^{3} G ;{ }^{4} D$ & 1.359 & \\
\hline & & 108219 & 71.69 & $3 d^{4} 4 d^{3} G ;{ }^{2} D$ & 6.9 & $3 d^{4} 4 d^{3} F ;{ }^{2} D$ & 1.193 & \\
\hline & & 108780 & 75.41 & $3 d^{4} 5 s^{3} D ;{ }^{2} D$ & 9.19 & $3 d^{4} 4 d^{3} F ;{ }^{2} D$ & 1.200 & \\
\hline & & 108875 & 33.65 & $3 d^{4} 4 d^{3} F ;{ }^{2} D$ & 23.46 & $3 d^{4} 5 s^{3} D ;{ }^{2} D$ & 1.191 & \\
\hline & & 109135 & 44.94 & $3 d^{4} 4 d^{3} G ;{ }^{2} F$ & 19.13 & $3 d^{4} 4 d^{3} P ;{ }^{2} F$ & 0.871 & \\
\hline & & 110092 & 20.48 & $3 d^{4} 4 d^{3} D ;{ }^{4} D$ & 19.71 & $3 d^{4} 4 d^{3} G ;{ }^{4} D$ & 1.352 & \\
\hline & & 110324 & 31.92 & $3 d^{4} 5 d^{5} D ;{ }^{4} F$ & 18.21 & $3 d^{4} 4 d^{3} D ;{ }^{4} F$ & 1.136 & \\
\hline & & 110431 & 21.12 & $3 d^{4} 5 d^{5} D ;{ }^{4} P$ & 17.86 & $3 d^{4} 4 d^{3} D ;{ }^{4} P$ & 1.505 & \\
\hline & & 110873 & 26.93 & $3 d^{4} 5 d^{5} D ;{ }^{4} G$ & 26.3 & $3 d^{4} 4 d^{3} G ;{ }^{4} G$ & 0.578 & \\
\hline & 109394.430 & 111469 & 80.84 & $3 d^{4} 5 d^{5} D ;{ }^{6} S$ & 15.35 & $3 d^{4} 6 d^{5} D ;{ }^{6} S$ & 2.002 & \\
\hline & & 112672 & 71.79 & $3 d^{4} 4 d^{3} D ;{ }^{4} F$ & 9 & $3 d^{4} 4 d^{3} \mathrm{G} ;{ }^{4} \mathrm{~F}$ & 1.018 & \\
\hline & & 112888 & 48.81 & $3 d^{4} 4 d^{3} D ;{ }^{4} G$ & 24.55 & $3 d^{4} 4 d^{3} D ;{ }^{2} F$ & 0.695 & \\
\hline & & 113025 & 37.65 & $3 d^{4} 4 d^{3} D ;{ }^{2} F$ & 30.86 & $3 d^{4} 4 d^{3} D ;{ }^{4} G$ & 0.749 & \\
\hline & & 113505 & 61.82 & $3 d^{4} 4 d^{3} D ;{ }^{4} P$ & 10.14 & $3 d^{4} 6 d^{5} D ;{ }^{4} P$ & 1.577 & \\
\hline & & 113851 & 36.13 & $3 d^{4} 5 s^{1} D ;{ }^{2} D$ & 21.94 & $3 d^{4} 4 d^{1} G ;{ }^{2} D$ & 1.214 & \\
\hline & & 113911 & 29.21 & $3 d^{4} 5 s^{1} D ;{ }^{2} D$ & 28.51 & $3 d^{4} 4 d^{3} D ;{ }^{4} D$ & 1.299 & \\
\hline & & 113972 & 20.76 & $3 d^{4} 4 d^{1} G ;{ }^{2} D$ & 18.56 & $3 d^{4} 4 d^{3} D ;{ }^{4} D$ & 1.281 & \\
\hline & & 114340 & 58.72 & $3 d^{4} 4 d^{3} D ;{ }^{2} D$ & 15.23 & $3 d^{4} 4 d^{1} s ;{ }^{2} D$ & 1.200 & \\
\hline & 115417.797 & 115416 & 96.77 & $3 d^{4} 7 s^{5} D ;{ }^{6} D$ & 3.05 & $3 d^{4} 7 s^{5} D ;{ }^{4} D$ & 1.650 & \\
\hline & 115818.391 & 115818 & 96.82 & $3 d^{4} 7 s^{5} D ;{ }^{4} D$ & 3.04 & $3 d^{4} 7 s^{5} D ;{ }^{6} D$ & 1.381 & \\
\hline & 116213.320 & 116227 & 75.63 & $3 d^{4} 6 d^{5} D ;{ }^{6} G$ & 15.12 & $3 d^{4} 6 d^{5} D ;{ }^{6} F$ & 0.996 & \\
\hline & & 116304 & 29.33 & $3 d^{4} 4 d^{1} G ;{ }^{2} F$ & 24.34 & $3 d^{4} 4 d^{3} D ;{ }^{2} F$ & 0.940 & \\
\hline & 116294.969 & 116308 & 38.81 & $3 d^{4} 6 d^{5} D ;{ }^{6} P$ & 30.27 & $3 d^{4} 6 d^{5} D ;{ }^{6} D$ & 1.534 & \\
\hline & 116477.406 & 116466 & 58.21 & $3 d^{4} 6 d^{5} D ;{ }^{6} F$ & 31.59 & $3 d^{4} 6 d^{5} D ;{ }^{6} P$ & 1.470 & \\
\hline & 116687.078 & 116670 & 58.12 & $3 d^{4} 6 d^{5} D ;{ }^{6} D$ & 21.07 & $3 d^{4} 6 d^{5} D ;{ }^{6} P$ & 1.636 & \\
\hline & 116877.070 & 116951 & 79.87 & $3 d^{4} 6 d^{5} D ;{ }^{4} G$ & 7.13 & $3 d^{4} 6 d^{5} D ;{ }^{4} F$ & 0.611 & \\
\hline & 117072.742 & 117156 & 55.39 & $3 d^{4} 4 d^{1} s ;{ }^{2} D$ & 13.83 & $3 d^{4} 4 d^{1} S ;{ }^{2} D$ & 1.213 & \\
\hline & 117228.406 & 117193 & 71.98 & $3 d^{4} 6 d^{5} D ;{ }^{4} F$ & 6.87 & $3 d^{4} 6 d^{5} D ;{ }^{4} G$ & 1.035 & \\
\hline & 117481.172 & 117400 & 58.37 & $3 d^{4} 6 d^{5} D ;{ }^{4} P$ & 13.7 & $3 d^{4} 6 d^{5} D ;{ }^{4} D$ & 1.499 & \\
\hline & & 117729 & 56.56 & $3 d^{4} 6 d^{5} D ;{ }^{4} D$ & 20.18 & $3 d^{4} 6 d^{5} D ;{ }^{4} P$ & 1.415 & \\
\hline & & 118772 & 99.38 & $3 \mathrm{~d}^{4} 6 \mathrm{~s}{ }^{1} \mathrm{~F} ;{ }^{2} \mathrm{~F}$ & 0.14 & $3 d^{4} 5 s^{3} F ;{ }^{4} F$ & 0.857 & \\
\hline & & 119678 & 66.44 & $3 d^{4} 4 d^{1} D ;{ }^{2} F$ & 17.53 & $3 d^{4} 4 d^{1} D ;{ }^{2} F$ & 0.865 & \\
\hline & & 119789 & 82.92 & $3 d^{4} 6 d^{5} D ;{ }^{6} S$ & 12.34 & $3 d^{4} 5 d^{5} D ;{ }^{6} S$ & 2.002 & \\
\hline & 117672.453 & 120129 & 64.41 & $3 d^{4} 4 d^{1} D ;{ }^{2} D$ & 15.96 & $3 d^{4} 4 d^{1} D ;{ }^{2} D$ & 1.193 & \\
\hline & 120870.633 & 120874 & 93.6 & $3 d^{4} 8 s^{5} D ;{ }^{6} D$ & 6.2 & $3 d^{4} 8 s^{5} D ;{ }^{4} D$ & 1.641 & \\
\hline & & 121156 & 93.73 & $3 d^{4} 8 s^{5} D ;{ }^{4} D$ & 6.2 & $3 d^{4} 8 s^{5} D ;{ }^{6} D$ & 1.390 & \\
\hline
\end{tabular}


Table 1 (continued)

\begin{tabular}{|c|c|c|c|c|c|c|c|c|}
\hline$J$-value & \multirow{2}{*}{$\begin{array}{c}\begin{array}{c}E_{\text {obs }} \\
\left(\mathrm{cm}^{-1}\right)\end{array} \\
{[8,27]} \\
12303.820\end{array}$} & \multirow{2}{*}{$\begin{array}{c}\begin{array}{c}E_{\text {calc }} \\
\left(\mathrm{cm}^{-1}\right)\end{array} \\
12263\end{array}$} & \multicolumn{2}{|c|}{$\begin{array}{l}\text { Largest eigenvalue component } \\
\text { (\%) }\end{array}$} & \multicolumn{2}{|c|}{$\begin{array}{l}\text { Next largest eigenvalue component } \\
(\%)\end{array}$} & Calc. $g_{J}$ & \multirow{2}{*}{$\begin{array}{c}\text { Obs. } g_{J} \\
{[5]} \\
1.578\end{array}$} \\
\hline & & & 99.89 & $3 d^{4} 4 s^{5} D ;{ }^{6} D$ & 0.03 & $3 d^{4} 5 s^{5} D ;{ }^{6} D$ & 1.589 & \\
\hline & 20024.012 & 20015 & 94.44 & $3 d^{4} 4 s^{5} D ;{ }^{4} D$ & 5.17 & $3 d^{54} D$ & 1.429 & 1.427 \\
\hline & 20517.793 & 20539 & 98.91 & $3 d^{54} G$ & 0.39 & $3 d^{4} 4 s^{3} G ;{ }^{4} G$ & 0.984 & 0.994 \\
\hline & 25033.668 & 25064 & 93.27 & $3 d^{54} D$ & 5.27 & $3 d^{4} 4 s^{5} D ;{ }^{4} D$ & 1.429 & 1.432 \\
\hline & 30156.732 & 30166 & 99.33 & $3 d^{4} 4 s^{3} \mathrm{H} ;{ }^{4} \mathrm{H}$ & 0.33 & $3 d^{4} 4 s^{3} G ;{ }^{4} G$ & 0.668 & 0.667 \\
\hline & 31168.576 & 31221 & 68.49 & $3 d^{4} 4 s^{3} F ;{ }^{4} F$ & 19.52 & $3 d^{4} 4 s^{3} F ;{ }^{4} F$ & 1.235 & 1.246 \\
\hline & 32355.656 & 32325 & 97.72 & $3 d^{52} F$ & 0.44 & $3 d^{4} 4 s^{3} F ;{ }^{2} F$ & 1.143 & \\
\hline & 32836.656 & 33002 & 84.5 & $3 d^{54} F$ & 7.03 & $3 d^{4} 4 s^{3} F ;{ }^{4} F$ & 1.233 & \\
\hline & 33521.090 & 33530 & 95.73 & $3 d^{4} 4 s^{3} G ;{ }^{4} G$ & 1.81 & $3 d^{4} 4 s^{3} F ;{ }^{4} F$ & 0.992 & 1.024 \\
\hline & 35607.512 & 35657 & 66.93 & $3 d^{4} 4 s^{3} F ;{ }^{2} F$ & 18.26 & $3 d^{4} 4 s^{3} F ;{ }^{2} F$ & 1.138 & 1.144 \\
\hline & 36101.527 & 36130 & 74.33 & $3 d^{52} G$ & 17.3 & $3 d^{4} 4 s^{3} G ;{ }^{2} G$ & 0.890 & \\
\hline & 38269.570 & 38302 & 99.28 & $3 d^{4} 4 s^{3} D ;{ }^{4} D$ & 0.48 & $3 d^{54} D$ & 1.429 & \\
\hline & 38508.934 & 38494 & 76.87 & $3 d^{4} 4 s^{3} G ;{ }^{2} G$ & 10.88 & $3 d^{52} G$ & 0.894 & 0.91 \\
\hline & 39683.719 & 39638 & 32.57 & $3 d^{4} 4 s^{1} G ;{ }^{2} G$ & 32.08 & $3 d^{52} F$ & 0.979 & \\
\hline & 39877.035 & 39823 & 52.62 & $3 d^{52} F$ & 20.25 & $3 d^{4} 4 s{ }^{1} G ;{ }^{2} G$ & 1.052 & \\
\hline & 50667.234 & 50611 & 98.44 & $3 d^{4} 4 s^{1} F ;{ }^{2} F$ & 0.57 & $3 d^{52} F$ & 1.144 & \\
\hline & 52297.766 & 52192 & 91.94 & $3 d^{52} G$ & 4.64 & $3 d^{3} 4 s^{22} G$ & 0.889 & \\
\hline & 53566.262 & 53572 & 97.59 & $3 d^{3} 4 s^{24} F$ & 2 & $3 d^{54} F$ & 1.238 & \\
\hline & 54887.986 & 54884 & 76.69 & $3 d^{4} 4 s^{3} F ;{ }^{4} F$ & 22.21 & $3 d^{4} 4 s^{3} F ;{ }^{4} F$ & 1.238 & \\
\hline & 59570.199 & 59595 & 76.87 & $3 d^{4} 4 s^{3} F ;{ }^{2} F$ & 21.95 & $3 d^{4} 4 s^{3} F ;{ }^{2} F$ & 1.142 & \\
\hline & \multirow{3}{*}{62701.734} & 62582 & 64.29 & $3 d^{4} 4 s{ }^{1} G ;{ }^{2} G$ & 34.24 & $3 d^{4} 4 s{ }^{1} G ;{ }^{2} G$ & 0.889 & \\
\hline & & 65898 & 94.52 & $3 d^{3} 4 s^{22} G$ & 4.65 & $3 d^{52} G$ & 0.889 & \\
\hline & & 82767 & 99.57 & $3 d^{3} 4 s^{22} F$ & 0.33 & $3 d^{52} F$ & 1.143 & \\
\hline & 83041.320 & 83033 & 99.57 & $3 d^{4} 5 s^{5} D ;{ }^{6} D$ & 0.16 & $3 d^{4} 5 s^{5} D ;{ }^{4} D$ & 1.588 & \\
\hline & 84726.680 & 84749 & 99.63 & $3 d^{4} 5 s^{5} D ;{ }^{4} D$ & 0.16 & $3 d^{4} 5 s^{5} D ;{ }^{6} D$ & 1.430 & \\
\hline & 86738.273 & 86646 & 98.21 & $3 d^{4} 4 d^{5} D ;{ }^{6} G$ & 1.09 & $3 d^{4} 4 d^{5} D ;{ }^{6} F$ & 1.146 & \\
\hline & 86782.047 & 86832 & 96.26 & $3 d^{4} 4 d^{5} D ;{ }^{6} P$ & 2.98 & $3 d^{4} 4 d^{5} D ;{ }^{6} D$ & 1.712 & \\
\hline & 87587.867 & 87530 & 77.43 & $3 d^{4} 4 d^{5} D ;{ }^{6} D$ & 19.02 & $3 d^{4} 4 d^{5} D ;{ }^{6} F$ & 1.554 & \\
\hline & 87858.469 & 87879 & 79.4 & $3 d^{4} 4 d^{5} D ;{ }^{6} F$ & 19.12 & $3 d^{4} 4 d^{5} D ;{ }^{6} D$ & 1.433 & \\
\hline & 89173.992 & 89297 & 96.22 & $3 d^{4} 4 d^{5} D ;{ }^{4} G$ & 1.23 & $3 d^{4} 4 d^{3} H ;{ }^{4} G$ & 0.986 & \\
\hline & 89885.008 & 89808 & 94.8 & $3 d^{4} 4 d^{5} D ;{ }^{4} D$ & 1.81 & $3 d^{4} 4 d^{5} D ;{ }^{4} F$ & 1.426 & \\
\hline & 90725.812 & 90799 & 93.16 & $3 d^{4} 4 d^{5} D ;{ }^{4} F$ & 1.87 & $3 d^{4} 4 d^{5} D ;{ }^{4} D$ & 1.241 & \\
\hline & 100068.859 & 100073 & 99.18 & $3 d^{4} 5 s^{3} \mathrm{H} ;{ }^{4} \mathrm{H}$ & 0.32 & $3 d^{4} 5 s^{3} G ;{ }^{4} G$ & 0.668 & \\
\hline & 101321.812 & 101276 & 78.98 & $3 d^{4} 5 s^{3} F ;{ }^{4} F$ & 19.25 & $3 d^{4} 5 s^{3} F ;{ }^{4} F$ & 1.235 & \\
\hline & \multirow[t]{2}{*}{102243.156} & 102225 & 78.7 & $3 d^{4} 5 s^{3} F ;{ }^{2} F$ & 18.72 & $3 d^{4} 5 s^{3} F ;{ }^{2} F$ & 1.138 & \\
\hline & & 103523 & 96.52 & $3 d^{4} 5 s^{3} G ;{ }^{4} G$ & 1.22 & $3 d^{4} 5 s^{3} F ;{ }^{4} F$ & 0.987 & \\
\hline & 103949.273 & 103709 & 94.86 & $3 \mathrm{~d}^{4} 4 \mathrm{~d}^{3} \mathrm{H} ;{ }^{4} \mathrm{H}$ & 2.88 & $3 d^{4} 4 d^{3} H ;{ }^{2} G$ & 0.676 & \\
\hline & & 104165 & 71.44 & $3 \mathrm{~d}^{4} 4 \mathrm{~d}^{3} \mathrm{H} ;{ }^{2} \mathrm{G}$ & 10.29 & $3 d^{4} 4 d^{3} G ;{ }^{2} G$ & 0.906 & \\
\hline & & 104363 & 72.38 & $3 d^{4} 4 d^{3} \mathrm{H} ;{ }^{4} \mathrm{~F}$ & 7.84 & $3 d^{4} 4 d^{3} G ;{ }^{4} F$ & 1.212 & \\
\hline & 104543.109 & 104453 & 94.46 & $3 d^{4} 5 s^{3} G ;{ }^{2} G$ & 1.89 & $3 d^{4} 4 d^{3} H ;{ }^{2} G$ & 0.895 & \\
\hline & 105198.992 & 104984 & 76.23 & $3 \mathrm{~d}^{4} 4 \mathrm{~d}^{3} \mathrm{H} ;{ }^{4} \mathrm{G}$ & 12.24 & $3 d^{4} 5 d^{5} D ;{ }^{4} G$ & 0.989 & \\
\hline & 105446.994 & 105436 & 99.42 & $3 d^{4} 6 s^{5} D ;{ }^{6} D$ & 0.45 & $3 d^{4} 6 s^{5} D ;{ }^{4} D$ & 1.588 & \\
\hline & & 105822 & 82.86 & $3 \mathrm{~d}^{4} 4 \mathrm{~d}^{3} \mathrm{H} ;{ }^{2} \mathrm{~F}$ & 7.56 & $3 d^{4} 4 d^{3} \mathrm{P} ;{ }^{2} \mathrm{~F}$ & 1.147 & \\
\hline & & 105857 & 66.47 & $3 d^{4} 4 d^{3} F ;{ }^{4} D$ & 11.9 & $3 d^{4} 4 d^{3} F ;{ }^{4} D$ & 1.421 & \\
\hline & & 106043 & 77.73 & $3 \mathrm{~d}^{4} 4 \mathrm{~d}^{3} \mathrm{~F} ;{ }^{4} \mathrm{H}$ & 15.05 & $3 d^{4} 4 d^{3} F ;{ }^{4} \mathrm{H}$ & 0.674 & \\
\hline & & 106097 & 38.56 & $3 d^{4} 4 d^{3} F ;{ }^{4} F$ & 31.01 & $3 d^{4} 4 d^{3} F ;{ }^{4} G$ & 1.115 & \\
\hline & 106030.914 & 106133 & 60.62 & $3 d^{4} 4 d^{3} F ;{ }^{4} G$ & 14.89 & $3 d^{4} 5 d^{5} D ;{ }^{4} G$ & 0.977 & \\
\hline & 106275.180 & 106286 & 21.23 & $3 d^{4} 4 d^{3} P ;{ }^{4} D$ & 18.61 & $3 d^{4} 4 d^{3} \mathrm{P} ;{ }^{4} \mathrm{~F}$ & 1.334 & \\
\hline & & 106575 & 38.38 & $3 d^{4} 4 d^{3} F ;{ }^{4} F$ & 13.26 & $3 d^{4} 4 d^{3} P ;{ }^{4} D$ & 1.303 & \\
\hline & 107006.266 & 107009 & 84.49 & $3 d^{4} 5 d^{5} D ;{ }^{6} G$ & 8.21 & $3 d^{4} 5 d^{5} D ;{ }^{6} F$ & 1.186 & \\
\hline & 107114.727 & 107089 & 68.73 & $3 d^{4} 5 d^{5} D ;{ }^{6} \mathrm{P}$ & 18.79 & $3 d^{4} 5 d^{5} D ;{ }^{6} D$ & 1.641 & \\
\hline & & 107125 & 48.25 & $3 d^{4} 4 d^{3} F ;{ }^{2} F$ & 9.95 & $3 d^{4} 4 d^{3} F ;{ }^{2} F$ & 1.092 & \\
\hline & & 107303 & 45.07 & $3 d^{4} 4 d^{3} G ;{ }^{4} D$ & 35.87 & $3 d^{4} 5 d^{5} D ;{ }^{4} D$ & 1.419 & \\
\hline & 107386.188 & 107349 & 43.49 & $3 d^{4} 5 d^{5} D ;{ }^{6} F$ & 16.27 & $3 d^{4} 5 d^{5} D ;{ }^{6} P$ & 1.424 & \\
\hline & & 107362 & 46.01 & $3 d^{4} 4 d^{3} F ;{ }^{2} G$ & 10.86 & $3 d^{4} 4 d^{3} F ;{ }^{2} G$ & 0.934 & \\
\hline & 107500.344 & 107395 & 51.21 & $3 d^{4} 4 d^{3} G ;{ }^{4} G$ & 27.58 & $3 d^{4} 5 d^{5} D ;{ }^{4} G$ & 1.013 & \\
\hline & & 107455 & 41.46 & $3 d^{4} 4 d^{3} G ;{ }^{4} F$ & 27.74 & $3 d^{4} 5 d^{5} D ;{ }^{4} F$ & 1.276 & \\
\hline & 107627.367 & 107624 & 56.18 & $3 d^{4} 5 d^{5} D ;{ }^{6} D$ & 34.07 & $3 d^{4} 5 d^{5} D ;{ }^{6} F$ & 1.526 & \\
\hline & 107716.266 & 107755 & 96.86 & $3 d^{4} 6 s^{5} D ;{ }^{4} D$ & 0.99 & $3 d^{4} 5 s^{3} D ;{ }^{4} D$ & 1.430 & \\
\hline & 107829.508 & 107825 & 82.64 & $3 d^{4} 4 d^{3} G ;{ }^{4} \mathrm{H}$ & 7.39 & $3 d^{4} 4 d^{3} F ;{ }^{2} G$ & 0.697 & \\
\hline & & 107892 & 94.26 & $3 d^{4} 5 s^{3} D ;{ }^{4} D$ & 2.92 & $3 d^{4} 4 d^{3} G ;{ }^{4} D$ & 1.429 & \\
\hline & & 108186 & 48.86 & $3 d^{4} 4 d^{3} G ;{ }^{2} F$ & 20.33 & $3 \mathrm{~d}^{4} 4 \mathrm{~d}^{3} \mathrm{P} ;{ }^{2} \mathrm{~F}$ & 1.141 & \\
\hline & & 108816 & 66.04 & $3 d^{4} 5 s^{1} G ;{ }^{2} G$ & 33.14 & $3 d^{4} 5 s^{1} G ;{ }^{2} G$ & 0.889 & \\
\hline & & 109400 & 39.21 & $3 d^{4} 4 d^{3} G ;{ }^{2} F$ & 25.05 & $3 d^{4} 4 d^{3} \mathrm{P} ;{ }^{2} \mathrm{~F}$ & 1.144 & \\
\hline & & 110276 & 20.19 & $3 d^{4} 4 d^{3} D ;{ }^{4} D$ & 17.12 & $3 d^{4} 4 d^{3} G ;{ }^{4} D$ & 1.358 & \\
\hline & 107850.555 & 110443 & 24.62 & $3 d^{4} 5 d^{5} D ;{ }^{4} F$ & 17.32 & $3 d^{4} 4 d^{3} D ;{ }^{4} F$ & 1.300 & \\
\hline & & 110647 & 63.26 & $3 d^{4} 4 d^{3} G ;{ }^{2} G$ & 7.07 & $3 d^{4} 4 d^{3} H ;{ }^{2} G$ & 0.894 & \\
\hline & & 110919 & 26.82 & $3 d^{4} 5 d^{5} D ;{ }^{4} G$ & 25.17 & $3 d^{4} 4 d^{3} G ;{ }^{4} G$ & 0.989 & \\
\hline & & 112697 & 56.6 & $3 d^{4} 4 d^{3} D ;{ }^{4} F$ & 7.84 & $3 d^{4} 4 d^{3} G ;{ }^{4} F$ & 1.170 & \\
\hline & & 112728 & 36.99 & $3 d^{4} 4 d^{1} \mathrm{I} ;{ }^{2} \mathrm{G}$ & 36.45 & $3 d^{4} 4 d^{3} D ;{ }^{2} G$ & 0.949 & \\
\hline
\end{tabular}


Table 1 (continued)

\begin{tabular}{|c|c|c|c|c|c|c|c|c|}
\hline$J$-value & $\begin{array}{c}E_{o b s} \\
\left(\mathrm{~cm}^{-1}\right) \\
{[8,27]}\end{array}$ & \multirow{2}{*}{$\begin{array}{c}E_{\text {calc }} \\
\left(\mathrm{cm}^{-1}\right)\end{array}$} & \multicolumn{2}{|c|}{$\begin{array}{c}\text { Largest eigenvalue component } \\
(\%)\end{array}$} & \multicolumn{2}{|c|}{$\begin{array}{l}\text { Next largest eigenvalue component } \\
(\%)\end{array}$} & Calc. $g_{J}$ & $\begin{array}{c}\text { Obs. } g_{J} \\
{[5]}\end{array}$ \\
\hline & & & 59.71 & $3 d^{4} 4 d^{3} D ;{ }^{4} G$ & 12.73 & $3 d^{4} 4 d^{3} D ;{ }^{2} F$ & 1.026 & \\
\hline & & 113033 & 45.83 & $3 d^{4} 4 d^{3} D ;{ }^{2} F$ & 16.96 & $3 d^{4} 4 d{ }^{1} G ;{ }^{2} F$ & 1.111 & \\
\hline & & 113896 & 55.78 & $3 d^{4} 4 d^{3} D ;{ }^{4} D$ & 16.76 & $3 d^{4} 6 d^{5} D ;{ }^{4} D$ & 1.429 & \\
\hline & & 113997 & 54.99 & $3 d^{4} 4 d^{1} G ;{ }^{2} G$ & 29.75 & $3 d^{4} 4 d^{1} G ;{ }^{2} G$ & 0.889 & \\
\hline & & 114951 & 43.04 & $3 d^{4} 4 d^{3} D ;{ }^{2} G$ & 41.96 & $3 d^{4} 4 d{ }^{1} \mathrm{I} ;{ }^{2} \mathrm{G}$ & 0.889 & \\
\hline & 115581.641 & 115580 & 97.78 & $3 d^{4} 7 s^{5} D ;{ }^{6} D$ & 2.04 & $3 d^{4} 7 s^{5} \mathrm{D} ;{ }^{4} \mathrm{D}$ & 1.585 & \\
\hline & 116047.820 & 116050 & 97.8 & $3 d^{4} 7 s^{5} D ;{ }^{4} D$ & 2.04 & $3 d^{4} 7 s^{5} D ;{ }^{6} D$ & 1.433 & \\
\hline & & 116203 & 32.75 & $3 d^{4} 4 d^{1} G ;{ }^{2} F$ & 29.59 & $3 d^{4} 4 d{ }^{3} D ;{ }^{2} F$ & 1.143 & \\
\hline & 116281.860 & 116288 & 68.03 & $3 d^{4} 6 d^{5} D ;{ }^{6} G$ & 20 & $3 d^{4} 6 d^{5} \mathrm{D} ;{ }^{6} \mathrm{~F}$ & 1.245 & \\
\hline & 116385.570 & 116384 & 39.96 & $3 d^{4} 6 d^{5} D ;{ }^{6} P$ & 33.36 & $3 d^{4} 6 d^{5} D ;{ }^{6} D$ & 1.538 & \\
\hline & 116572.391 & 116550 & 51.7 & $3 d^{4} 8 s^{5} D ;{ }^{6} F$ & 35.86 & $3 d^{4} 6 d^{5} D ;{ }^{6} P$ & 1.492 & \\
\hline & 116790.258 & 116762 & 56.46 & $3 d^{4} 6 d^{5} D ;{ }^{6} D$ & 21.91 & $3 d^{4} 6 d^{5} \mathrm{D} ;{ }^{6} \mathrm{~F}$ & 1.567 & \\
\hline & 116985.359 & 117046 & 77.99 & $3 d^{4} 6 d^{5} D ;{ }^{4} G$ & 9.46 & $3 d^{4} 6 d^{5} D ;{ }^{4} F$ & 1.013 & \\
\hline & 117263.375 & 117323 & 73.62 & $3 d^{4} 6 d^{5} D ;{ }^{4} D$ & 6.41 & $3 d^{4} 4 d^{3} D ;{ }^{4} D$ & 1.416 & \\
\hline & 117520.656 & 117754 & 74.44 & $3 d^{4} 6 d^{5} D ;{ }^{4} F$ & 9.38 & $3 d^{4} 6 d^{5} D ;{ }^{4} G$ & 1.225 & \\
\hline & & 118774 & 99.41 & $3 d^{4} 5 s^{1} F ;{ }^{2} F$ & 0.19 & $3 d^{4} 5 s{ }^{3} F ;{ }^{4} F$ & 1.144 & \\
\hline & & 119716 & 68.84 & $3 d^{4} 4 d^{1} D ;{ }^{2} F$ & 18.21 & $3 d^{4} 4 d^{1} D ;{ }^{2} F$ & 1.143 & \\
\hline & & 120271 & 72.88 & $3 d^{4} 4 d^{1} D ;{ }^{2} G$ & 17.16 & $3 d^{4} 4 d^{1} D ;{ }^{2} G$ & 0.889 & \\
\hline & 121036.359 & 121037 & 96.08 & $3 d^{4} 8 s^{5} D ;{ }^{6} D$ & 3.74 & $3 d^{4} 8 s^{5} D ;{ }^{4} D$ & 1.583 & \\
\hline & & 121384 & 96.17 & $3 d^{4} 8 s^{5} D ;{ }^{4} D$ & 3.73 & $3 d^{4} 8 s^{5} D ;{ }^{6} D$ & 1.435 & \\
\hline & & 121997 & 99.52 & $3 d^{4} 6 s{ }^{3} \mathrm{H} ;{ }^{4} \mathrm{H}$ & 0.15 & $3 d^{4} 6 s^{3} G ;{ }^{4} G$ & 0.667 & \\
\hline & & 122153 & 97.47 & $3 d^{4} 5 d^{3} \mathrm{H} ;{ }^{4} \mathrm{H}$ & 0.77 & $3 d^{4} 6 d^{3} \mathrm{H} ;{ }^{4} \mathrm{H}$ & 0.669 & \\
\hline & & 122482 & 47.65 & $3 d^{4} 4 d^{1} F ;{ }^{2} F$ & 45.69 & $3 d^{4} 5 d^{3} \mathrm{H} ;{ }^{2} \mathrm{~F}$ & 1.143 & \\
\hline & & 122805 & 78.67 & $3 d^{4} 5 d^{3} H ;{ }^{2} G$ & 8.59 & $3 d^{4} 4 d^{1} F ;{ }^{2} G$ & 0.896 & \\
\hline & & 123077 & 83.86 & $3 d^{4} 5 d^{3} \mathrm{H} ;{ }^{4} \mathrm{~F}$ & 4.07 & $3 d^{4} 5 d^{3} G ;{ }^{4} F$ & 1.230 & \\
\hline & & 123109 & 75.96 & $3 d^{4} 4 d^{1} F ;{ }^{2} G$ & 8.22 & $3 d^{4} 5 d^{3} \mathrm{H} ;{ }^{2} \mathrm{G}$ & 0.889 & \\
\hline & & 123607 & 18.57 & $3 d^{4} 5 d^{3} P ;{ }^{4} F$ & 17.16 & $3 d^{4} 5 d^{3} \mathrm{H} ;{ }^{4} \mathrm{G}$ & 1.163 & \\
\hline & & 123628 & 44.91 & $3 d^{4} 5 d^{3} H ;{ }^{4} G$ & 16.47 & $3 d^{4} 5 d^{3} F ;{ }^{4} G$ & 1.050 & \\
\hline & & 123950 & 19.24 & $3 d^{4} 5 d^{3} P ;{ }^{4} F$ & 15.68 & $3 d^{4} 5 d^{3} \mathrm{H} ;{ }^{2} \mathrm{~F}$ & 1.205 & \\
\hline & 124310.148 & 124309 & 30.49 & $3 d^{4} 9 s^{5} D ;{ }^{6} D$ & 17.39 & $3 d^{4} 6 s^{3} P ;{ }^{4} D$ & 1.445 & \\
\hline & & 124310 & 64.06 & $3 d^{4} 9 s^{5} D ;{ }^{6} D$ & 8.2 & $3 d^{4} 6 s^{3} P ;{ }^{4} D$ & 1.516 & \\
\hline \multicolumn{9}{|c|}{ 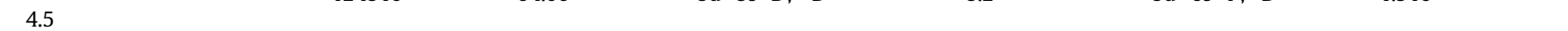 } \\
\hline & 12496.456 & 12467 & 99.87 & $3 d^{4} 4 s^{5} D ;{ }^{6} D$ & 0.04 & $3 d^{4} 4 s^{3} F ;{ }^{4} F$ & 1.557 & 1.554 \\
\hline & 20519.270 & 20545 & 98.97 & $3 d^{54} G$ & 0.39 & $3 d^{4} 4 s^{3} G ;{ }^{4} G$ & 1.172 & 1.161 \\
\hline & 30218.779 & 30239 & 99.19 & $3 d^{4} 4 s^{3} \mathrm{H} ;{ }^{4} \mathrm{H}$ & 0.55 & $3 d^{4} 4 s^{3} G ;{ }^{4} G$ & 0.971 & 0.978 \\
\hline & 31219.336 & 31276 & 68.16 & $3 d^{4} 4 s{ }^{3} F ;{ }^{4} F$ & 18.87 & $3 d^{4} 4 s^{3} F ;{ }^{4} F$ & 1.331 & 1.34 \\
\hline & 32854.246 & 33014 & 84.12 & $3 d^{54} F$ & 7.86 & $3 d^{4} 4 s^{3} F ;{ }^{4} F$ & 1.331 & \\
\hline & 33618.941 & 33638 & 95.91 & $3 d^{4} 4 s{ }^{3} G ;{ }^{4} G$ & 1.61 & $3 d^{4} 4 s^{3} F ;{ }^{4} F$ & 1.174 & 1.185 \\
\hline & 34630.914 & 34627 & 68.03 & $3 d^{4} 4 s^{3} \mathrm{H} ;{ }^{2} \mathrm{H}$ & 28.06 & $3 d^{52} H$ & 0.916 & \\
\hline & 35610.305 & 35591 & 65.67 & $3 d^{52} \mathrm{H}$ & 30.53 & $3 d^{4} 4 s^{3} \mathrm{H} ;{ }^{2} \mathrm{H}$ & 0.914 & \\
\hline & 36272.523 & 36264 & 73.45 & $3 d^{52} G$ & 14.67 & $3 d^{4} 4 s^{3} G ;{ }^{2} G$ & 1.103 & \\
\hline & 38563.008 & 38559 & 74.32 & $3 d^{4} 4 s^{3} G ;{ }^{2} G$ & 12.32 & $3 d^{4} 4 s^{1} G ;{ }^{2} G$ & 1.111 & 1.1 \\
\hline & 39824.371 & 39778 & 47.56 & $3 d^{4} 4 s{ }^{1} G ;{ }^{2} G$ & 26.93 & $3 d^{4} 4 s{ }^{1} G ;{ }^{2} G$ & 1.111 & \\
\hline & 52321.023 & 52196 & 92.1 & $3 d^{52} G$ & 4.46 & $3 d^{3} 4 s^{22} G$ & 1.112 & \\
\hline & 53923.523 & 53942 & 97.14 & $3 d^{3} 4 s^{24} F$ & 1.92 & $3 d^{54} F$ & 1.333 & \\
\hline & 54867.512 & 54842 & 77.26 & $3 d^{4} 4 s^{3} F ;{ }^{4} F$ & 21.76 & $3 d^{4} 4 s^{3} F ;{ }^{4} F$ & 1.334 & \\
\hline & 62688.961 & 62579 & 64.55 & $3 d^{4} 4 s^{1} G ;{ }^{2} G$ & 34.36 & $3 d^{4} 4 s^{1} G ;{ }^{2} G$ & 1.112 & \\
\hline & & 66194 & 93.6 & $3 d^{3} 4 s^{22} G$ & 4.4 & $3 d^{52} G$ & 1.109 & \\
\hline & & 69833 & 98 & $3 d^{3} 4 s^{22} H$ & 1.22 & $3 d^{3} 4 s^{22} G$ & 0.911 & \\
\hline & 83240.422 & 83241 & 99.69 & $3 d^{4} 5 s^{5} D ;{ }^{6} D$ & 0.1 & $3 d^{4} 4 d^{5} D ;{ }^{6} D$ & 1.557 & \\
\hline & 86847.008 & 86946 & 98.1 & $3 d^{4} 4 d^{5} D ;{ }^{6} G$ & 1.24 & $3 d^{4} 4 d^{5} D ;{ }^{6} F$ & 1.275 & \\
\hline & 87687.508 & 87629 & 83.8 & $3 d^{4} 4 d^{5} D ;{ }^{6} D$ & 15.34 & $3 d^{4} 4 d^{5} \mathrm{D} ;{ }^{6} \mathrm{~F}$ & 1.537 & \\
\hline & 87948.531 & 87981 & 82.98 & $3 d^{4} 4 d^{5} D ;{ }^{6} F$ & 15.7 & $3 d^{4} 4 d^{5} D ;{ }^{6} D$ & 1.453 & \\
\hline & 89325.281 & 89463 & 96.29 & $3 d^{4} 4 d^{5} D ;{ }^{4} G$ & 1.25 & $3 d^{4} 4 d^{3} \mathrm{H} ;{ }^{4} \mathrm{G}$ & 1.173 & \\
\hline & 90850.961 & 90940 & 95.03 & $3 d^{4} 4 d^{5} D ;{ }^{4} F$ & 1.24 & $3 d^{4} 4 d^{3} \mathrm{H} ;{ }^{4} \mathrm{~F}$ & 1.333 & \\
\hline & 100135.781 & 100146 & 98.63 & $3 d^{4} 5 s^{3} H ;{ }^{4} \mathrm{H}$ & 0.55 & $3 d^{4} 5 s^{3} G ;{ }^{4} G$ & 0.971 & \\
\hline & 101021.797 & 101050 & 97.05 & $3 d^{4} 5 s^{3} \mathrm{H} ;{ }^{2} \mathrm{H}$ & 1.03 & $3 d^{4} 5 s{ }^{3} F ;{ }^{4} F$ & 0.917 & \\
\hline & 101382.938 & 101334 & 78.26 & $3 d^{4} 5 s^{3} F ;{ }^{4} F$ & 18.49 & $3 d^{4} 5 s^{3} \mathrm{~F} ;{ }^{4} \mathrm{~F}$ & 1.325 & \\
\hline & 103627.039 & 103630 & 97.29 & $3 d^{4} 5 s^{3} G ;{ }^{4} G$ & 1.08 & $3 d^{4} 5 s{ }^{3} F ;{ }^{4} F$ & 1.173 & \\
\hline & 103755.383 & 103685 & 88.57 & $3 d^{4} 4 d^{3} \mathrm{H} ;{ }^{4} \mathrm{I}$ & 6.31 & $3 d^{4} 4 d^{3} \mathrm{H} ;{ }^{4} \mathrm{H}$ & 0.744 & \\
\hline & 104023.961 & 103797 & 90.9 & $3 d^{4} 4 d^{3} \mathrm{H} ;{ }^{4} \mathrm{H}$ & 6.16 & $3 d^{4} 4 d^{3} \mathrm{H} ;{ }^{4} \mathrm{I}$ & 0.956 & \\
\hline & & 104192 & 65.91 & $3 d^{4} 4 d^{3} H ;{ }^{2} G$ & 10.97 & $3 d^{4} 4 d^{3} G ;{ }^{2} G$ & 1.137 & \\
\hline & & 104463 & 68.26 & $3 d^{4} 4 d^{3} \mathrm{H} ;{ }^{4} \mathrm{~F}$ & 10.15 & $3 d^{4} 4 d^{3} \mathrm{H} ;{ }^{2} \mathrm{G}$ & 1.301 & \\
\hline & 104666.359 & 104652 & 76.75 & $3 d^{4} 4 d^{3} \mathrm{H} ;{ }^{4} \mathrm{G}$ & 11.59 & $3 d^{4} 5 d^{5} D ;{ }^{4} G$ & 1.174 & \\
\hline & 105255.305 & 105089 & 97.43 & $3 d^{4} 5 s^{3} G ;{ }^{2} G$ & 0.78 & $3 d^{4} 4 d^{3} \mathrm{H} ;{ }^{2} \mathrm{G}$ & 1.111 & \\
\hline & 105365.508 & 105269 & 75.66 & $3 d^{4} 4 d^{3} \mathrm{H} ;{ }^{2} \mathrm{H}$ & 14.16 & $3 d^{4} 4 d^{3} F ;{ }^{2} H$ & 0.913 & \\
\hline & 105650.000 & 106045 & 46.53 & $3 d^{4} 4 d^{3} F ;{ }^{4} F$ & 20.53 & $3 d^{4} 4 d{ }^{3} P ;{ }^{4} F$ & 1.330 & \\
\hline & & 106090 & 72.85 & $3 d^{4} 4 d^{3} F ;{ }^{4} H$ & 18.33 & $3 d^{4} 4 d^{3} F ;{ }^{4} H$ & 0.971 & \\
\hline & 106148.734 & 106220 & 60.76 & $3 d^{4} 4 d^{3} F ;{ }^{4} G$ & 17.57 & $3 d^{4} 5 d^{5} D ;{ }^{4} G$ & 1.165 & \\
\hline & & 106597 & 39.66 & $3 d^{4} 4 d^{3} P ;{ }^{4} F$ & 27.51 & $3 d^{4} 4 d^{3} F ;{ }^{4} F$ & 1.331 & \\
\hline
\end{tabular}


Table 1 (continued)

\begin{tabular}{|c|c|c|c|c|c|c|c|c|}
\hline \multirow[t]{2}{*}{$J$-value } & \multirow{2}{*}{$\begin{array}{c}E_{o b s} \\
\left(\mathrm{~cm}^{-1}\right) \\
{[8,27]}\end{array}$} & \multirow{2}{*}{$\begin{array}{c}E_{\text {calc }} \\
\left(\mathrm{cm}^{-1}\right)\end{array}$} & \multicolumn{2}{|c|}{$\begin{array}{c}\text { Largest eigenvalue component } \\
(\%)\end{array}$} & \multicolumn{2}{|c|}{$\begin{array}{c}\text { Next largest eigenvalue component } \\
(\%)\end{array}$} & Calc. $g_{J}$ & $\begin{array}{c}\text { Obs. } g_{\text {J }} \\
{[5]}\end{array}$ \\
\hline & & & 99.86 & $3 d^{4} 6 s^{5} \mathrm{D} ;{ }^{6} \mathrm{D}$ & 0.08 & $3 d^{4} 5 s^{5} D ;{ }^{6} D$ & 1.557 & \\
\hline & 107111.761 & 107122 & 88.73 & $3 d^{4} 5 d^{5} D ;{ }^{6} G$ & 7.94 & $3 d^{4} 5 d^{5} D ;{ }^{6} F$ & 1.287 & \\
\hline & & 107341 & 38.96 & $3 d^{4} 4 d{ }^{3} F ;{ }^{2} G$ & 11.14 & $3 d^{4} 4 d^{3} F ;{ }^{2} \mathrm{H}$ & 1.050 & \\
\hline & 107455.484 & 107422 & 42.71 & $3 d^{4} 5 d^{5} D ;{ }^{6} D$ & 40.03 & $3 d^{4} 5 d^{5} D ;{ }^{6} F$ & 1.457 & \\
\hline & 107632.227 & 107505 & 27.96 & $3 d^{4} 4 d^{3} G ;{ }^{4} G$ & 14.75 & $3 d^{4} 5 d^{5} D ;{ }^{4} G$ & 1.084 & \\
\hline & & 107520 & 30.28 & $3 d^{4} 4 d^{3} G ;{ }^{4} G$ & 13.78 & $3 d^{4} 4 d^{3} F ;{ }^{2} \mathrm{H}$ & 1.093 & \\
\hline & & 107584 & 40.51 & $3 d^{4} 4 d{ }^{3} G ;{ }^{4} F$ & 27.49 & $3 d^{4} 5 d^{5} D ;{ }^{4} F$ & 1.278 & \\
\hline & 107696.273 & 107688 & 51.28 & $3 d^{4} 5 d^{5} D ;{ }^{6} D$ & 45.84 & $3 d^{4} 5 d^{5} D ;{ }^{6} F$ & 1.495 & \\
\hline & 107706.742 & 107780 & 43.06 & $3 d^{4} 4 d^{3} G ;{ }^{4} \mathrm{H}$ & 34.58 & $3 d^{4} 4 d^{3} G ;{ }^{4} I$ & 0.875 & \\
\hline & 107922.453 & 107946 & 46.4 & $3 d^{4} 4 d^{3} G ;{ }^{4} \mathrm{H}$ & 36.9 & $3 d^{4} 4 d^{3} G ;{ }^{4} I$ & 0.874 & \\
\hline & & 108814 & 66.1 & $3 d^{4} 5 s^{1} G ;{ }^{2} G$ & 33.16 & $3 d^{4} 5 s{ }^{1} G ;{ }^{2} G$ & 1.112 & \\
\hline & & 109086 & 70.51 & $3 d^{4} 4 d^{3} G ;{ }^{2} H$ & 10.57 & $3 d^{4} 4 d^{1} \mathrm{I} ;{ }^{2} \mathrm{H}$ & 0.914 & \\
\hline & & 110491 & 38.59 & $3 d^{4} 5 d^{5} D ;{ }^{4} F$ & 27.12 & $3 d^{4} 4 d^{3} D ;{ }^{4} F$ & 1.329 & \\
\hline & & 110798 & 55.72 & $3 d^{4} 4 d^{3} G ;{ }^{2} G$ & 9.09 & $3 d^{4} 4 d^{1} G ;{ }^{2} G$ & 1.116 & \\
\hline & & 110967 & 26.14 & $3 d^{4} 5 d^{5} D ;{ }^{4} G$ & 23.35 & $3 d^{4} 4 d^{3} G ;{ }^{4} G$ & 1.172 & \\
\hline & & 112684 & 37.26 & $3 d^{4} 4 d^{3} D ;{ }^{2} G$ & 35.89 & $3 d^{4} 4 d^{1} I ;{ }^{2} G$ & 1.142 & \\
\hline & & 112762 & 52.41 & $3 d^{4} 4 d^{3} D ;{ }^{4} F$ & 9.05 & $3 d^{4} 4 d^{3} G ;{ }^{4} F$ & 1.285 & \\
\hline & & 112797 & 54.49 & $3 \mathrm{~d}^{4} 4 \mathrm{~d}^{1} \mathrm{I} ;{ }^{2} \mathrm{H}$ & 26.43 & $3 d^{4} 4 d^{1} G ;{ }^{2} H$ & 0.917 & \\
\hline & & 112951 & 72.71 & $3 d^{4} 4 d^{3} D ;{ }^{4} G$ & 7.12 & $3 d^{4} 5 d^{5} D ;{ }^{4} G$ & 1.183 & \\
\hline & & 114095 & 51 & $3 d^{4} 4 d{ }^{1} G ;{ }^{2} G$ & 28.48 & $3 d^{4} 4 d^{1} G ;{ }^{2} G$ & 1.108 & \\
\hline & & 114853 & 31.18 & $3 d^{4} 4 d^{1} G ;{ }^{2} \mathrm{H}$ & 22.65 & $3 d^{4} 4 d{ }^{1} \mathrm{I} ;{ }^{2} \mathrm{H}$ & 0.940 & \\
\hline & & 114958 & 37.03 & $3 d^{4} 4 d^{1} \mathrm{I} ;{ }^{2} \mathrm{G}$ & 36.52 & $3 d^{4} 4 d^{3} D ;{ }^{2} G$ & 1.084 & \\
\hline & 115788.281 & 115789 & 99.79 & $3 d^{4} 7 s^{5} D ;{ }^{6} D$ & 0.08 & $3 d^{4} 8 s^{5} D ;{ }^{6} D$ & 1.557 & \\
\hline & 116388.859 & 116388 & 76.91 & $3 d^{4} 6 d^{5} D ;{ }^{6} G$ & 18.65 & $3 d^{4} 6 d^{5} D ;{ }^{6} F$ & 1.312 & \\
\hline & 116601.500 & 116577 & 41.43 & $3 d^{4} 6 d^{5} D ;{ }^{6} D$ & 38.69 & $3 d^{4} 6 d^{5} D ;{ }^{6} F$ & 1.455 & \\
\hline & 116831.570 & 116795 & 54.62 & $3 d^{4} 6 d^{5} D ;{ }^{6} D$ & 41.53 & $3 d^{4} 6 d^{5} D ;{ }^{6} F$ & 1.497 & \\
\hline & 117141.492 & 117185 & 80.77 & $3 d^{4} 6 d^{5} D ;{ }^{4} G$ & 7.12 & $3 d^{4} 6 d^{5} D ;{ }^{4} F$ & 1.186 & \\
\hline & 117488.422 & 117492 & 82.77 & $3 d^{4} 6 d^{5} D ;{ }^{4} F$ & 7.03 & $3 d^{4} 6 d^{5} D ;{ }^{4} G$ & 1.322 & \\
\hline & & 120264 & 74 & $3 d^{4} 4 d^{1} D ;{ }^{2} G$ & 17.55 & $3 d^{4} 4 d^{1} D ;{ }^{2} G$ & 1.112 & \\
\hline & 121246.773 & 121247 & 99.78 & $3 d^{4} 8 s^{5} D ;{ }^{6} D$ & 0.08 & $3 d^{4} 7 s^{5} D ;{ }^{6} D$ & 1.557 & \\
\hline & & 122055 & 97.86 & $3 d^{4} 6 s^{3} \mathrm{H} ;{ }^{4} \mathrm{H}$ & 1.68 & $3 d^{4} 6 s^{3} \mathrm{H} ;{ }^{2} \mathrm{H}$ & 0.969 & \\
\hline & & 122136 & 87.3 & $3 d^{4} 5 d^{3} \mathrm{H} ;{ }^{4} \mathrm{I}$ & 10.35 & $3 d^{4} 5 d^{3} \mathrm{H} ;{ }^{4} \mathrm{H}$ & 0.754 & \\
\hline & & 122238 & 87.49 & $3 d^{4} 5 d^{3} \mathrm{H} ;{ }^{4} \mathrm{H}$ & 10.31 & $3 d^{4} 5 d^{3} \mathrm{H} ;{ }^{4} \mathrm{I}$ & 0.945 & \\
\hline & & 122473 & 97.76 & $3 d^{4} 6 s^{3} \mathrm{H} ;{ }^{2} \mathrm{H}$ & 1.7 & $3 d^{4} 6 s^{3} \mathrm{H} ;{ }^{4} \mathrm{H}$ & 0.911 & \\
\hline & & 122883 & 73.27 & $3 d^{4} 5 d^{3} \mathrm{H} ;{ }^{2} \mathrm{G}$ & 7.6 & $3 d^{4} 5 d^{3} \mathrm{H} ;{ }^{2} \mathrm{H}$ & 1.100 & \\
\hline & & 123112 & 50.62 & $3 d^{4} 4 d{ }^{1} F ;{ }^{2} G$ & 18.74 & $3 d^{4} 5 d^{3} H ;{ }^{4} F$ & 1.121 & \\
\hline & & 123170 & 60.55 & $3 d^{4} 5 d^{3} \mathrm{H} ;{ }^{4} \mathrm{~F}$ & 23.47 & $3 d^{4} 4 d{ }^{1} F ;{ }^{2} G$ & 1.266 & \\
\hline & & 123226 & 40.51 & $3 d^{4} 5 d^{3} \mathrm{H} ;{ }^{2} \mathrm{H}$ & 22.36 & $3 d^{4} 4 d^{1} F ;{ }^{2} \mathrm{H}$ & 0.977 & \\
\hline & & 123701 & 61.11 & $3 d^{4} 5 d^{3} H ;{ }^{4} G$ & 23.54 & $3 d^{4} 5 d^{3} F ;{ }^{4} G$ & 1.174 & \\
\hline & & 123999 & 59.93 & $3 d^{4} 4 d{ }^{1} F ;{ }^{2} \mathrm{H}$ & 16.95 & $3 d^{4} 5 d^{3} F ;{ }^{2} \mathrm{H}$ & 0.910 & \\
\hline & & 124254 & 50.39 & $3 d^{4} 5 d^{3} P ;{ }^{4} F$ & 33.72 & $3 d^{4} 5 d^{3} P ;{ }^{4} F$ & 1.333 & \\
\hline & & 124417 & 76.67 & $3 d^{4} 6 s^{3} F ;{ }^{4} F$ & 20.3 & $3 d^{4} 6 s^{3} F ;{ }^{4} F$ & 1.330 & \\
\hline & 124523.727 & 124517 & 74.3 & $3 d^{4} 5 d^{3} F ;{ }^{4} \mathrm{H}$ & 22.22 & $3 d^{4} 5 d^{3} F ;{ }^{4} \mathrm{H}$ & 0.967 & \\
\hline & & 124524 & 99.82 & $3 d^{4} 9 s^{5} \mathrm{D} ;{ }^{6} \mathrm{D}$ & 0.07 & $3 d^{4} 8 s^{5} D ;{ }^{6} D$ & 1.557 & \\
\hline & & 124537 & 67.28 & $3 d^{4} 5 s^{3} F ;{ }^{4} F$ & 15.53 & $3 d^{4} 4 d^{3} F ;{ }^{4} F$ & 1.333 & \\
\hline & & 124791 & 63.6 & $3 d^{4} 5 d^{3} F ;{ }^{4} F$ & 16.28 & $3 d^{4} 5 d^{3} F ;{ }^{4} F$ & 1.328 & \\
\hline \multicolumn{9}{|c|}{ ( } \\
\hline & 20512.098 & 20544 & 98.97 & $3 d^{54} G$ & 0.38 & $3 d^{4} 4 s^{3} G ;{ }^{4} G$ & 1.273 & 1.278 \\
\hline & 30143.246 & 30099 & 98.84 & $3 d^{52} I$ & 0.45 & $3 \mathrm{~d}^{52} \mathrm{H}$ & 0.924 & \\
\hline & 30298.471 & 30333 & 99.36 & $3 d^{4} 4 s^{3} \mathrm{H} ;{ }^{4} \mathrm{H}$ & 0.48 & $3 d^{4} 4 s^{3} \mathrm{G} ;{ }^{4} \mathrm{G}$ & 1.134 & 1.234 \\
\hline & 33694.141 & 33721 & 97.57 & $3 d^{4} 4 s^{3} G ;{ }^{4} G$ & 1.29 & $3 d^{4} 4 s^{3} H ;{ }^{2} \mathrm{H}$ & 1.270 & 1.276 \\
\hline & 34812.930 & 34812 & 63 & $3 d^{4} 4 s^{3} \mathrm{H} ;{ }^{2} \mathrm{H}$ & 34.55 & $3 d^{52} \mathrm{H}$ & 1.093 & \\
\hline & 35707.473 & 35700 & 62.95 & $3 d^{52} \mathrm{H}$ & 35.34 & $3 d^{4} 4 s^{3} \mathrm{H} ;{ }^{2} \mathrm{H}$ & 1.091 & \\
\hline & 40228.293 & 40215 & 99.61 & $3 d^{4} 4 s^{1} \mathrm{I} ;{ }^{2} \mathrm{I}$ & 0.23 & $3 d^{4} 4 s^{3} \mathrm{H} ;{ }^{2} \mathrm{H}$ & 0.923 & \\
\hline & & 70068 & 99.26 & $3 d^{3} 4 s^{22} H$ & 0.7 & $3 d^{52} \mathrm{H}$ & 1.091 & \\
\hline & 86980.078 & 87088 & 98.47 & $3 d^{4} 4 d^{5} D ;{ }^{6} G$ & 0.91 & $3 d^{4} 4 d^{5} D ;{ }^{6} F$ & 1.344 & \\
\hline & 88001.336 & 88060 & 98.58 & $3 d^{4} 4 d^{5} D ;{ }^{6} F$ & 0.92 & $3 d^{4} 4 d^{5} D ;{ }^{6} G$ & 1.454 & \\
\hline & 89508.499 & 89667 & 96.63 & $3 d^{4} 4 d^{5} D ;{ }^{4} G$ & 1.28 & $3 d^{4} 4 d^{3} H ;{ }^{4} G$ & 1.274 & \\
\hline & 100221.594 & 100240 & 98.9 & $3 d^{4} 5 s^{3} \mathrm{H} ;{ }^{4} \mathrm{H}$ & 0.5 & $3 d^{4} 5 s^{3} G ;{ }^{4} G$ & 1.134 & \\
\hline & 101194.789 & 101240 & 98.94 & $3 d^{4} 5 s^{3} \mathrm{H} ;{ }^{2} \mathrm{H}$ & 0.51 & $3 d^{4} 5 s^{3} \mathrm{H} ;{ }^{4} \mathrm{H}$ & 1.092 & \\
\hline & 103737.000 & 103746 & 98.84 & $3 d^{4} 5 s^{3} G ;{ }^{4} G$ & 0.45 & $3 d^{4} 5 s^{3} \mathrm{H} ;{ }^{4} \mathrm{H}$ & 1.272 & \\
\hline & 103843.312 & 103775 & 85.39 & $3 d^{4} 4 d^{3} \mathrm{H} ;{ }^{4} \mathrm{I}$ & 9.97 & $3 d^{4} 4 d^{3} \mathrm{H} ;{ }^{4} \mathrm{H}$ & 0.983 & \\
\hline & & 103893 & 88.53 & $3 d^{4} 4 d^{3} \mathrm{H} ;{ }^{4} \mathrm{H}$ & 9.56 & $3 d^{4} 4 d^{3} \mathrm{H} ;{ }^{4} \mathrm{I}$ & 1.116 & \\
\hline & 104460.164 & 104435 & 98.5 & $3 d^{4} 4 d^{3} \mathrm{H} ;{ }^{4} \mathrm{~K}$ & 0.33 & $3 d^{4} 5 d^{3} \mathrm{H} ;{ }^{4} \mathrm{~K}$ & 0.771 & \\
\hline & 105423.344 & 105209 & 77.79 & $3 d^{4} 4 d^{3} \mathrm{H} ;{ }^{4} \mathrm{G}$ & 10.85 & $3 d^{4} 5 d^{5} D ;{ }^{4} G$ & 1.273 & \\
\hline & & 105425 & 76.32 & $3 d^{4} 4 d^{3} \mathrm{H} ;{ }^{2} \mathrm{H}$ & 15.39 & $3 d^{4} 4 d^{3} F ;{ }^{2} H$ & 1.091 & \\
\hline & 106145.227 & 105984 & 81.51 & $3 d^{4} 4 d^{3} \mathrm{H} ;{ }^{2} \mathrm{I}$ & 6.61 & $3 d^{4} 4 d^{1} \mathrm{I} ;{ }^{2} \mathrm{I}$ & 0.935 & \\
\hline & & 106161 & 73.86 & $3 d^{4} 4 d^{3} F ;{ }^{4} \mathrm{H}$ & 18.04 & $3 d^{4} 4 d^{3} F ;{ }^{4} \mathrm{H}$ & 1.125 & \\
\hline & 106293.438 & 106326 & 60.79 & $3 d^{4} 4 d{ }^{3} F ;{ }^{4} G$ & 19.45 & $3 d^{4} 5 d^{5} D ;{ }^{4} G$ & 1.265 & \\
\hline & 107246.797 & 107268 & 92.17 & $3 d^{4} 5 d^{5} D ;{ }^{6} G$ & 5.41 & $3 d^{4} 5 d^{5} D ;{ }^{6} F$ & 1.349 & \\
\hline
\end{tabular}


Table 1 (continued)

\begin{tabular}{|c|c|c|c|c|c|c|c|c|}
\hline \multirow[t]{20}{*}{$J$-value } & \multirow{2}{*}{$\begin{array}{c}E_{o b s} \\
\left(\mathrm{~cm}^{-1}\right) \\
{[8,27]}\end{array}$} & \multirow{2}{*}{$\begin{array}{c}E_{\text {calc }} \\
\left(\mathrm{cm}^{-1}\right)\end{array}$} & \multicolumn{2}{|c|}{$\begin{array}{l}\text { Largest eigenvalue component } \\
\qquad(\%)\end{array}$} & \multicolumn{2}{|c|}{$\begin{array}{l}\text { Next largest eigenvalue component } \\
(\%)\end{array}$} & Calc. $g_{J}$ & \multirow{2}{*}{$\begin{array}{c}\text { Obs. } g_{J} \\
{[5]}\end{array}$} \\
\hline & & & 31.05 & $3 d^{4} 4 d^{3} F ;{ }^{2} H$ & 21.96 & $3 d^{4} 4 d^{3} G ;{ }^{4} G$ & 1.142 & \\
\hline & 107701.281 & 107677 & 50.1 & $3 d^{4} 5 d^{5} D ;{ }^{6} F$ & 17.6 & $3 d^{4} 4 d^{3} G ;{ }^{4} G$ & 1.330 & \\
\hline & & 107695 & 43.28 & $3 d^{4} 5 d^{5} D ;{ }^{6} F$ & 26.81 & $3 d^{4} 4 d^{3} G ;{ }^{4} G$ & 1.319 & \\
\hline & 107760.703 & 107825 & 42.48 & $3 d^{4} 4 d^{3} G ;{ }^{4} I$ & 36.99 & $3 d^{4} 4 d^{3} G ;{ }^{4} H$ & 1.052 & \\
\hline & 108017.984 & 108032 & 54.18 & $3 d^{4} 4 d^{3} G ;{ }^{4} H$ & 35.98 & $3 d^{4} 4 d^{3} G ;{ }^{4} I$ & 1.068 & \\
\hline & & 108761 & 80.93 & $3 d^{4} 5 s^{1} I^{2}{ }^{2} \mathrm{I}$ & 15.73 & $3 d^{4} 4 d^{3} G ;{ }^{2} I$ & 0.924 & \\
\hline & & 108999 & 69.47 & $3 d^{4} 4 d^{3} G ;{ }^{2} I$ & 18.77 & $3 d^{4} 5 s{ }^{1} \mathrm{I} ;{ }^{2} \mathrm{I}$ & 0.927 & \\
\hline & & 109210 & 70.55 & $3 d^{4} 4 d^{3} G ;{ }^{2} \mathrm{H}$ & 10.87 & $3 \mathrm{~d}^{4} 4 \mathrm{~d}^{1} \mathrm{I} ;{ }^{2} \mathrm{H}$ & 1.090 & \\
\hline & & 110986 & 30.22 & $3 d^{4} 5 d^{5} D ;{ }^{4} G$ & 24.08 & $3 d^{4} 4 d^{3} G ;{ }^{4} G$ & 1.273 & \\
\hline & & 112827 & 53.21 & $3 d^{4} 4 d^{1}{ }^{1} ;{ }^{2} \mathrm{H}$ & 28.34 & $3 d^{4} 4 d^{1} G ;{ }^{2} H$ & 1.093 & \\
\hline & & 112941 & 77.45 & $3 d^{4} 4 d^{3} D ;{ }^{4} G$ & 8.44 & $3 d^{4} 5 d^{5} D ;{ }^{4} G$ & 1.272 & \\
\hline & & 113274 & 44.92 & $3 d^{4} 4 d^{1} G ;{ }^{2} I$ & 30.97 & $3 \mathrm{~d}^{4} 4 \mathrm{~d}^{1} \mathrm{I} ;{ }^{2} \mathrm{I}$ & 0.924 & \\
\hline & & 114519 & 35.6 & $3 d^{4} 4 d^{1} I^{2}{ }^{2} I$ & 15.66 & $3 d^{4} 4 d^{1} G ;{ }^{2} I$ & 0.957 & \\
\hline & & 114940 & 28.91 & $3 d^{4} 4 d^{1}{ }^{1} ;{ }^{2} \mathrm{H}$ & 21.28 & $3 d^{4} 4 d^{1} \mathrm{I} ;{ }^{2} \mathrm{H}$ & 1.058 & \\
\hline & 116531.117 & 116523 & 86.34 & $3 d^{4} 6 d^{5} D ;{ }^{6} G$ & 12.18 & $3 d^{4} 6 d^{5} D ;{ }^{6} F$ & 1.357 & \\
\hline & 116828.922 & 116783 & 86.77 & $3 d^{4} 6 d^{5} D ;{ }^{6} F$ & 12.31 & $3 d^{4} 6 d^{5} D ;{ }^{6} G$ & 1.441 & \\
\hline & 117342.328 & 117368 & 88.08 & $3 d^{4} 6 d^{5} D ;{ }^{4} G$ & 4.58 & $3 d^{4} 5 d^{3} H ;{ }^{4} G$ & 1.274 & \\
\hline & & 122134 & 98.35 & $3 \mathrm{~d}^{4} 6 \mathrm{~s}^{3} \mathrm{H} ;{ }^{4} \mathrm{H}$ & 1.28 & $3 \mathrm{~d}^{4} 6 \mathrm{~s}^{3} \mathrm{H} ;{ }^{2} \mathrm{H}$ & 1.133 & \\
\hline & & 122215 & 83.19 & $3 d^{4} 5 d^{3} \mathrm{H} ;{ }^{4} \mathrm{I}$ & 13.6 & $3 d^{4} 5 d^{3} \mathrm{H} ;{ }^{4} \mathrm{H}$ & 0.987 & \\
\hline \multicolumn{9}{|l|}{6.5} \\
\hline & 30149.879 & 30124 & 99.28 & $3 d^{52} I$ & 0.26 & $3 d^{4} 4 d^{3} \mathrm{H} ;{ }^{2} \mathrm{I}$ & 1.077 & \\
\hline & 30391.832 & 30444 & 99.79 & $3 d^{4} 4 s^{3} \mathrm{H} ;{ }^{4} \mathrm{H}$ & 0.08 & $3 d^{4} 4 s^{1} \mathrm{I} ;{ }^{2} \mathrm{I}$ & 1.231 & 1.234 \\
\hline & 40202.109 & 40209 & 99.78 & $3 d^{4} 4 s^{1} \mathrm{I} ;{ }^{2} \mathrm{I}$ & 0.08 & $3 \mathrm{~d}^{4} 4 \mathrm{~s}^{3} \mathrm{H} ;{ }^{4} \mathrm{H}$ & 1.077 & \\
\hline & 87137.023 & 87258 & 99.38 & $3 d^{4} 4 d^{5} D ;{ }^{6} G$ & 0.45 & $3 d^{4} 5 d^{5} D ;{ }^{6} G$ & 1.385 & \\
\hline & 100322.078 & 100355 & 99.77 & $3 d^{4} 5 s^{3} H ;{ }^{4} \mathrm{H}$ & 0.11 & $3 d^{4} 5 s^{1} \mathrm{I} ;{ }^{2} \mathrm{I}$ & 1.231 & \\
\hline & 103948.250 & 103887 & 83.68 & $3 \mathrm{~d}^{4} 4 \mathrm{~d}^{3} \mathrm{H} ;{ }^{4} \mathrm{I}$ & 12.14 & $3 \mathrm{~d}^{4} 4 \mathrm{~d}^{3} \mathrm{H} ;{ }^{4} \mathrm{H}$ & 1.123 & \\
\hline & 104190.523 & 103993 & 86.46 & $3 \mathrm{~d}^{4} 4 \mathrm{~d}^{3} \mathrm{H} ;{ }^{4} \mathrm{H}$ & 11.79 & $3 \mathrm{~d}^{4} 4 \mathrm{~d}^{3} \mathrm{H} ;{ }^{4} \mathrm{I}$ & 1.216 & \\
\hline & 104539.984 & 104521 & 97.82 & $3 \mathrm{~d}^{4} 4 \mathrm{~d}^{3} \mathrm{H} ;{ }^{4} \mathrm{~K}$ & 0.82 & $3 \mathrm{~d}^{4} 4 \mathrm{~d}^{3} \mathrm{H} ;{ }^{2} \mathrm{~K}$ & 0.965 & \\
\hline & 105124.836 & 105151 & 97.95 & $3 \mathrm{~d}^{4} 4 \mathrm{~d}^{3} \mathrm{H} ;{ }^{2} \mathrm{~K}$ & 0.83 & $3 d^{4} 4 d^{3} \mathrm{H} ;{ }^{4} \mathrm{~K}$ & 0.935 & \\
\hline & 106342.898 & 106180 & 71.17 & $3 \mathrm{~d}^{4} 4 \mathrm{~d}^{3} \mathrm{H} ;{ }^{2} \mathrm{I}$ & 13.03 & $3 d^{4} 4 d^{3} \mathrm{~F} ;{ }^{4} \mathrm{H}$ & 1.104 & \\
\hline & & 106245 & 64.04 & $3 d^{4} 4 d^{3} F ;{ }^{4} H$ & 15.12 & $3 d^{4} 4 d^{3} F ;{ }^{4} \mathrm{H}$ & 1.201 & \\
\hline & 107412.031 & 107448 & 98.46 & $3 d^{4} 5 d^{5} D ;{ }^{6} G$ & 0.97 & $3 d^{4} 7 s^{5} D ;{ }^{6} G$ & 1.385 & \\
\hline & 107846.664 & 107906 & 61.85 & $3 d^{4} 4 d^{3} G ;{ }^{4} I$ & 35.41 & $3 d^{4} 4 d^{3} G ;{ }^{4} H$ & 1.151 & \\
\hline & 108103.953 & 108112 & 61.9 & $3 d^{4} 4 d^{3} G ;{ }^{4} \mathrm{H}$ & 32.03 & $3 d^{4} 4 d^{3} G ;{ }^{4} I$ & 1.188 & \\
\hline & & 108784 & 94.85 & $3 d^{4} 5 s^{1} \mathrm{I} ;{ }^{2} \mathrm{I}$ & 3.91 & $3 d^{4} 4 d^{3} G ;{ }^{2} I$ & 1.077 & \\
\hline & & 109219 & 80.93 & $3 d^{4} 4 d^{3} G ;{ }^{2} I$ & 10.65 & $3 d^{4} 4 d^{1} \mathrm{I} ;{ }^{2} \mathrm{I}$ & 1.077 & \\
\hline & & 112233 & 99.34 & $3 \mathrm{~d}^{4} 4 \mathrm{~d}^{1} \mathrm{I} ;{ }^{2} \mathrm{~K}$ & 0.3 & $3 d^{4} 5 d^{1} \mathrm{I} ;{ }^{2} \mathrm{~K}$ & 0.934 & \\
\hline & & 113315 & 47.09 & $3 d^{4} 4 d^{1} G ;{ }^{2} I$ & 27.69 & $3 d^{4} 4 d^{1} \mathrm{I} ;{ }^{2} \mathrm{I}$ & 1.077 & \\
\hline & & 114562 & 46.34 & $3 d^{4} 4 d^{1} \mathrm{I} ;{ }^{2} \mathrm{I}$ & 17.97 & $3 d^{4} 4 d{ }^{1} G ;{ }^{2} I$ & 1.077 & \\
\hline & 116708.656 & 116695 & 98.86 & $3 d^{4} 7 s^{5} D ;{ }^{6} G$ & 1.01 & $3 d^{4} 5 d^{5} D ;{ }^{6} G$ & 1.385 & \\
\hline & & 122230 & 99.81 & $3 \mathrm{~d}^{4} 6 \mathrm{~s}^{3} \mathrm{H} ;{ }^{4} \mathrm{H}$ & 0.1 & $3 d^{4} 6 s{ }^{1} \mathrm{I} ;{ }^{2} \mathrm{I}$ & 1.231 & \\
\hline \multicolumn{9}{|c|}{ 然 } \\
\hline & 104069.906 & 104028 & 96.31 & $3 d^{4} 4 d^{3} \mathrm{H} ;{ }^{4} \mathrm{I}$ & 2.87 & $3 d^{4} 4 d^{3} G ;{ }^{4} I$ & 1.200 & \\
\hline & 104633.094 & 104624 & 98.23 & $3 \mathrm{~d}^{4} 4 \mathrm{~d}^{3} \mathrm{H} ;{ }^{4} \mathrm{~K}$ & 0.66 & $3 \mathrm{~d}^{4} 4 \mathrm{~d}^{3} \mathrm{H} ;{ }^{2} \mathrm{~K}$ & 1.091 & \\
\hline & 105285.422 & 105337 & 98.8 & $3 \mathrm{~d}^{4} 4 \mathrm{~d}^{3} \mathrm{H} ;{ }^{2} \mathrm{~K}$ & 0.71 & $3 \mathrm{~d}^{4} 4 \mathrm{~d}^{3} \mathrm{H} ;{ }^{4} \mathrm{~K}$ & 1.067 & \\
\hline & 107981.719 & 108065 & 95.95 & $3 d^{4} 4 d^{3} G ;{ }^{4} I$ & 2.85 & $3 \mathrm{~d}^{4} 4 \mathrm{~d}^{3} \mathrm{H} ;{ }^{4} \mathrm{I}$ & 1.200 & \\
\hline & & 112245 & 99.47 & $3 d^{4} 4 d^{1} \mathrm{I} ;{ }^{2} \mathrm{~K}$ & 0.3 & $3 d^{4} 5 d^{1} \mathrm{I} ;{ }^{2} \mathrm{~K}$ & 1.067 & \\
\hline & & 113013 & 99.56 & $3 d^{4} 4 d^{1} \mathrm{I} ;{ }^{2} \mathrm{~L}$ & 0.24 & $3 d^{4} 5 d^{1} \mathrm{I} ;{ }^{2} \mathrm{~L}$ & 0.941 & \\
\hline \multicolumn{9}{|c|}{ 然 } \\
\hline & 104734.508 & 104744 & 99.46 & $3 \mathrm{~d}^{4} 4 \mathrm{~d}^{3} \mathrm{H} ;{ }^{4} \mathrm{~K}$ & 0.33 & $3 d^{4} 5 d^{3} \mathrm{H} ;{ }^{4} \mathrm{~K}$ & 1.177 & \\
\hline & & 113033 & 99.58 & $3 \mathrm{~d}^{4} 4 \mathrm{~d}^{1} \mathrm{I} ;{ }^{2} \mathrm{~L}$ & 0.24 & $3 \mathrm{~d}^{4} 5 \mathrm{~d}^{1} \mathrm{I} ;{ }^{2} \mathrm{~L}$ & 1.059 & \\
\hline
\end{tabular}


Table 2

Values of the intra-configuration fine structure parameters for $\mathrm{Cr}$ II even configurations.

\begin{tabular}{|c|c|c|}
\hline Configuration & Parameter & $\begin{array}{l}\text { Value } \\
\left(\mathrm{cm}^{-1}\right)\end{array}$ \\
\hline $3 d^{5}$ & $\begin{array}{l}E_{a v} \\
\mathrm{~F}^{2}(3 \mathrm{~d}, 3 \mathrm{~d}) \\
\mathrm{F}^{4}(3 \mathrm{~d}, 3 \mathrm{~d}) \\
\zeta_{3 d} \\
\alpha \\
\beta\end{array}$ & $\begin{array}{r}34907(13) \\
54319(62) \\
31973(71) \\
199(23) \\
52(1) \\
-83(19)\end{array}$ \\
\hline $3 d^{4} 4 s$ & $\begin{array}{l}E_{a v} \\
\mathrm{~F}^{2}(3 \mathrm{~d}, 3 \mathrm{~d}) \\
\mathrm{F}^{4}(3 \mathrm{~d}, 3 \mathrm{~d}) \\
\mathrm{G}^{2}(3 \mathrm{~d}, 4 \mathrm{~s}) \\
\zeta_{3 d} \\
\alpha \\
\beta \\
\mathrm{T}_{s}\end{array}$ & $\begin{array}{r}39185(8) \\
59200(40) \\
35099(61) \\
7044(27) \\
231(11) \\
58(2) \\
-125(23) \\
21(9)\end{array}$ \\
\hline $3 d^{3} 4 s^{2}$ & $\begin{array}{l}E_{a v} \\
\mathrm{~F}^{2}(3 \mathrm{~d}, 3 \mathrm{~d}) \\
\mathrm{F}^{4}(3 \mathrm{~d}, 3 \mathrm{~d}) \\
\zeta_{3 d} \\
\alpha \\
\beta\end{array}$ & $\begin{array}{r}68847(23) \\
63138(89) \\
37001(98) \\
257(21) \\
21(2) \\
0^{f}\end{array}$ \\
\hline $3 d^{4} 5 s$ & $\begin{array}{l}E_{a v} \\
\mathrm{~F}^{2}(3 \mathrm{~d}, 3 \mathrm{~d}) \\
\mathrm{F}^{4}(3 \mathrm{~d}, 3 \mathrm{~d}) \\
\mathrm{G}^{2}(3 \mathrm{~d}, 5 \mathrm{~s}) \\
\zeta_{3 d} \\
\alpha \\
\beta \\
\mathrm{T}_{s}\end{array}$ & $\begin{array}{r}107550(11) \\
59996(59) \\
37827(69) \\
1448(30) \\
234^{d} \\
65(2) \\
0^{f} \\
29(7)\end{array}$ \\
\hline $3 d^{4} 6 s$ & $\begin{array}{l}E_{a v} \\
\mathrm{~F}^{2}(3 \mathrm{~d}, 3 \mathrm{~d}) \\
\mathrm{F}^{4}(3 \mathrm{~d}, 3 \mathrm{~d}) \\
\mathrm{G}^{2}(3 \mathrm{~d}, 6 \mathrm{~s}) \\
\zeta_{3 d} \\
\alpha \\
\beta\end{array}$ & $\begin{array}{r}129035(20) \\
60565^{d} \\
37986^{d} \\
723(42) \\
235^{d} \\
0^{f} \\
0^{f}\end{array}$ \\
\hline $3 d^{4} 7 s$ & $\begin{array}{l}E_{a v} \\
\mathrm{~F}^{2}(3 \mathrm{~d}, 3 \mathrm{~d}) \\
\mathrm{F}^{4}(3 \mathrm{~d}, 3 \mathrm{~d}) \\
\mathrm{G}^{2}(3 \mathrm{~d}, 7 \mathrm{~s}) \\
\zeta_{3 d} \\
\alpha \\
\beta\end{array}$ & $\begin{array}{r}139030(21) \\
60583^{d} \\
38050^{d} \\
270^{d} \\
236^{d} \\
0^{f} \\
0^{f}\end{array}$ \\
\hline $3 d^{4} 8 s$ & $\begin{array}{l}E_{a v} \\
\mathrm{~F}^{2}(3 \mathrm{~d}, 3 \mathrm{~d}) \\
\mathrm{F}^{4}(3 \mathrm{~d}, 3 \mathrm{~d}) \\
\mathrm{G}^{2}(3 \mathrm{~d}, 8 \mathrm{~s}) \\
\zeta_{3 d} \\
\alpha \\
\beta\end{array}$ & $\begin{array}{r}144504(30) \\
60853^{d} \\
37500^{d} \\
148^{d} \\
236^{d} \\
0^{f} \\
0^{f}\end{array}$ \\
\hline $3 d^{4} 9 s$ & $\begin{array}{l}E_{a v} \\
\mathrm{~F}^{2}(3 \mathrm{~d}, 3 \mathrm{~d}) \\
\mathrm{F}^{4}(3 \mathrm{~d}, 3 \mathrm{~d}) \\
\mathrm{G}^{2}(3 \mathrm{~d}, 9 \mathrm{~s}) \\
\zeta_{3 d} \\
\alpha \\
\beta\end{array}$ & $\begin{array}{r}144523(43) \\
60853^{d} \\
37500^{d} \\
92^{d} \\
236^{d} \\
0^{f} \\
0^{f}\end{array}$ \\
\hline $3 d^{4} 4 d$ & $\begin{array}{l}E_{a v} \\
\mathrm{~F}^{2}(3 \mathrm{~d}, 3 \mathrm{~d}) \\
\mathrm{F}^{4}(3 \mathrm{~d}, 3 \mathrm{~d}) \\
\zeta_{3 d} \\
\zeta_{4 d} \\
\alpha\end{array}$ & $\begin{array}{r}112817(22) \\
59397(56) \\
40284(119) \\
244(11) \\
10^{d} \\
46(2)\end{array}$ \\
\hline
\end{tabular}


Table 2 (continued)

\begin{tabular}{|c|c|c|}
\hline Configuration & Parameter & $\begin{array}{l}\text { Value } \\
\left(\mathrm{cm}^{-1}\right)\end{array}$ \\
\hline & $\begin{array}{l}\beta \\
\mathrm{F}^{2}(3 \mathrm{~d}, 4 \mathrm{~d}) \\
\mathrm{F}^{4}(3 \mathrm{~d}, 4 \mathrm{~d}) \\
\mathrm{G}^{0}(3 \mathrm{~d}, 4 \mathrm{~d}) \\
\mathrm{G}^{2}(3 \mathrm{~d}, 4 \mathrm{~d}) \\
\mathrm{G}^{4}(3 \mathrm{~d}, 4 \mathrm{~d})\end{array}$ & $\begin{array}{r}-52^{d} \\
4266(74) \\
1600(88) \\
1694(12) \\
1297(67) \\
619(67)\end{array}$ \\
\hline $3 d^{4} 5 d$ & $\begin{array}{l}E_{a v} \\
\mathrm{~F}^{2}(3 \mathrm{~d}, 3 \mathrm{~d}) \\
\mathrm{F}^{4}(3 \mathrm{~d}, 3 \mathrm{~d}) \\
\zeta_{3 d} \\
\zeta_{5 d} \\
\alpha \\
\beta \\
\mathrm{F}^{2}(3 \mathrm{~d}, 5 \mathrm{~d}) \\
\mathrm{F}^{4}(3 \mathrm{~d}, 5 \mathrm{~d}) \\
\mathrm{G}^{0}(3 \mathrm{~d}, 5 \mathrm{~d}) \\
\mathrm{G}^{2}(3 \mathrm{~d}, 5 \mathrm{~d}) \\
\mathrm{G}^{4}(3 \mathrm{~d}, 5 \mathrm{~d})\end{array}$ & $\begin{array}{r}131497(14) \\
60812^{d} \\
37673^{d} \\
244^{d} \\
4^{d} \\
0^{f} \\
0^{f} \\
1621^{d} \\
785^{d} \\
931^{d} \\
763^{d} \\
520^{d}\end{array}$ \\
\hline $3 d^{4} 6 d$ & $\begin{array}{l}E_{a v} \\
\mathrm{~F}^{2}(3 \mathrm{~d}, 3 \mathrm{~d}) \\
\mathrm{F}^{4}(3 \mathrm{~d}, 3 \mathrm{~d}) \\
\zeta_{3 d} \\
\zeta_{6 d} \\
\alpha \\
\beta \\
\mathrm{F}^{2}(3 \mathrm{~d}, 6 \mathrm{~d}) \\
\mathrm{F}^{4}(3 \mathrm{~d}, 6 \mathrm{~d}) \\
\mathrm{G}^{0}(3 \mathrm{~d}, 6 \mathrm{~d}) \\
\mathrm{G}^{2}(3 \mathrm{~d}, 6 \mathrm{~d}) \\
\mathrm{G}^{4}(3 \mathrm{~d}, 6 \mathrm{~d})\end{array}$ & $\begin{array}{r}140071(11) \\
60583^{d} \\
37500^{d} \\
244^{d} \\
2^{d} \\
0^{f} \\
0^{f} \\
799^{d} \\
354^{d} \\
403^{d} \\
383^{d} \\
267^{d}\end{array}$ \\
\hline
\end{tabular}

${ }^{f}$ Frozen to zero.

${ }^{d}$ Deduced from $a b$ initio or fs calculations. 
Table 3

Values of even configuration interaction parameters.

\begin{tabular}{|c|c|c|}
\hline Configurations & Parameter $^{a}$ & $\begin{array}{l}\text { Value } \\
\left(\mathrm{cm}^{-1}\right)\end{array}$ \\
\hline $3 d^{5}-3 d^{3} 4 s^{2}$ & $\mathrm{R}^{2}$ & $11076(194)$ \\
\hline $3 d^{5}-3 d^{4} 4 s$ & $\mathrm{R}^{2}$ & $-3710(55)$ \\
\hline $3 d^{5}-3 d^{4} 5 s$ & $\mathrm{R}^{2}$ & $-2799(450)$ \\
\hline $3 d^{5}-3 d^{4} 4 d$ & $\begin{array}{l}\mathrm{R}^{2} \\
\mathrm{R}^{4}\end{array}$ & $\begin{array}{l}9075(279) \\
7754(354)\end{array}$ \\
\hline $3 d^{5}-3 d^{4} 5 d$ & $\begin{array}{l}\mathrm{R}^{2} \\
\mathrm{R}^{4}\end{array}$ & $\begin{array}{l}3736(462) \\
2445(480)\end{array}$ \\
\hline $3 d^{5}-3 d^{4} 6 d$ & $\begin{array}{l}\mathrm{R}^{2} \\
\mathrm{R}^{4}\end{array}$ & $\begin{array}{l}2892(499) \\
3168(700)\end{array}$ \\
\hline $3 d^{4} 4 s-3 d^{3} 4 s^{2}$ & $\mathrm{R}^{2}$ & $-897(350)$ \\
\hline $3 d^{4} 4 s-3 d^{4} 5 s$ & $\mathrm{E}^{2}$ & $2911(478)$ \\
\hline $3 d^{4} 4 s-3 d^{4} 6 s$ & $\mathrm{E}^{2}$ & $2053(500)$ \\
\hline $3 d^{4} 4 s-3 d^{4} 4 d$ & $\begin{array}{l}\mathrm{D}^{2} \\
\mathrm{E}^{2}\end{array}$ & $\begin{array}{l}6514(266) \\
2097(391)\end{array}$ \\
\hline $3 d^{4} 4 s-3 d^{4} 5 d$ & $\mathrm{D}^{2}$ & $2657(498)$ \\
\hline
\end{tabular}

${ }^{a}$ For meaning of $\mathrm{R}^{2}, \mathrm{D}^{2}, \mathrm{E}^{2}$ and $\mathrm{R}^{4}$ see Cowan's book [28]. 
Table 4

Proposals of new positions for the quartet $3 d^{4}\left({ }^{5} D\right) 5 d{ }^{4} F$

\begin{tabular}{lll}
\hline$J$-value & $\begin{array}{l}\text { Position given in [27] } \\
\left(\mathrm{cm}^{-1}\right)\end{array}$ & $\begin{array}{l}\text { Our proposed position } \\
\left(\mathrm{cm}^{-1}\right)\end{array}$ \\
\hline 1.5 & 107516.711 & 110256 \\
2.5 & 107726.773 & 110324 \\
3.5 & 107850.552 & 110443 \\
4.5 & 107947.996 & 110491 \\
\hline
\end{tabular}


Table 5

Comparison of the experimental and calculated energy values, percentages of first and second components and $g_{J}$-factors for Cr II odd-parity configurations.

\begin{tabular}{|c|c|c|c|c|c|c|c|c|}
\hline$J$-value & $\begin{array}{c}E_{o b s} \\
\left(\mathrm{~cm}^{-1}\right) \\
{[8,27]}\end{array}$ & $\begin{array}{c}E_{\text {calc }} \\
\left(\mathrm{cm}^{-1}\right)\end{array}$ & \multicolumn{2}{|c|}{$\begin{array}{l}\text { Largest eigenvalue component } \\
(\%)\end{array}$} & \multicolumn{2}{|c|}{$\begin{array}{c}\text { Next largest eigenvalue component } \\
(\%)\end{array}$} & \multirow[t]{2}{*}{ Calc. $g_{J}$} & \multirow{2}{*}{$\begin{array}{c}\text { Obs. } g_{J} \\
\text { [5] }\end{array}$} \\
\hline \multicolumn{7}{|l|}{0.5} & & \\
\hline & 46823.305 & 46811 & 99.48 & $3 d^{4} 4 p^{5} \mathrm{D} ;{ }^{6} \mathrm{~F}$ & 0.24 & $3 d^{4} 5 p^{5} D ;{ }^{6} F$ & -0.669 & -0.689 \\
\hline & 48749.277 & 48685 & 79.02 & $3 d^{4} 4 p^{5} \mathrm{D} ;{ }^{4} \mathrm{P}$ & 18.02 & $3 d^{4} 4 p^{5} D ;{ }^{6} D$ & 2.790 & 2.844 \\
\hline & 49492.711 & 49455 & 81.70 & $3 d^{4} 4 p^{5} D ;{ }^{6} D$ & 17.72 & $3 d^{4} 4 p^{5} D ;{ }^{4} P$ & 3.217 & 3.155 \\
\hline & 54417.957 & 54369 & 97.15 & $3 d^{4} 4 p^{5} D ;{ }^{4} D$ & 0.89 & $3 d^{4} 4 p^{3} p ;{ }^{4} D$ & -0.002 & 0.007 \\
\hline & 63801.754 & 63839 & 48.15 & $3 d^{4} 4 p^{3} p ;{ }^{2} S$ & 42.74 & $3 d^{4} 4 p^{3} p ;{ }^{2} S$ & 1.951 & \\
\hline & 65029.336 & 64748 & 48.95 & $3 d^{4} 4 p^{3} P ;{ }^{4} D$ & 34.21 & $3 d^{4} 4 p^{3} p ;{ }^{4} D$ & 0.021 & \\
\hline & 66256.438 & 66425 & 52.96 & $3 d^{4} 4 p^{3} P ;{ }^{4} P$ & 34.21 & $3 d^{4} 4 p^{3} p ;{ }^{4} P$ & 2.540 & 2.545 \\
\hline & 66871.828 & 66711 & 53.48 & $3 d^{4} 4 p^{3} \mathrm{P} ;{ }^{2} \mathrm{P}$ & 27.84 & $3 d^{4} 4 p^{3} p ;{ }^{2} P$ & 0.819 & \\
\hline & 67859.562 & 68024 & 65.08 & $3 d^{4} 4 p^{3} F ;{ }^{4} D$ & 19.78 & $3 d^{4} 4 p^{3} F ;{ }^{4} D$ & 0.003 & \\
\hline & 73406.891 & 73552 & 95.14 & $3 d^{4} 4 p^{3} D ;{ }^{4} D$ & 1.05 & $3 d^{4} 4 p^{3} D ;{ }^{2} P$ & 0.016 & \\
\hline & 74920.383 & 74592 & 93.54 & $3 d^{4} 4 p^{3} D ;{ }^{4} P$ & 3.65 & $3 \mathrm{~d}^{4} 4 \mathrm{p}^{3} \mathrm{P} ;{ }^{4} \mathrm{P}$ & 2.622 & \\
\hline & 74853.852 & 74836 & 50.96 & $3 d^{4} 4 p^{3} \mathrm{D} ;{ }^{2} \mathrm{P}$ & 32.63 & $3 d^{4} 4 p{ }^{1} s ;{ }^{2} P$ & 0.697 & \\
\hline & 77777.320 & 77681 & 36.24 & $3 d^{4} 4 p{ }^{1} s ;{ }^{2} P$ & 34.51 & $3 d^{4} 4 p^{3} D ;{ }^{2} P$ & 0.666 & \\
\hline & 81648.641 & 81602 & 95.09 & $\begin{array}{l}3 \mathrm{~d}^{3} 4 \mathrm{~s} 4 \mathrm{p} \\
{ }^{4} \mathrm{~F} \cdot{ }^{3} \mathrm{P} ;{ }^{6} \mathrm{D}\end{array}$ & 2.28 & $3 d^{3} 4 s 4 p^{4}$ P. ${ }^{3} P ;{ }^{6} D$ & 3.258 & \\
\hline & 81734.648 & 81733 & 97.20 & $\begin{array}{l}3 d^{3} 4 s 4 p \\
{ }^{4} F^{3} \mathrm{P} ;{ }^{6} \mathrm{~F}\end{array}$ & 1.87 & $3 d^{3} 4 s 4 p^{4} F \cdot{ }^{3} P ;{ }^{6} D$ & -0.589 & \\
\hline & 82853.891 & 82762 & 68.50 & $3 d^{4} 4 p^{1} D ;{ }^{2} P$ & 15.36 & $3 d^{4} 4 p{ }^{1} D ;{ }^{2} P$ & 0.667 & \\
\hline & 85486.164 & 85333 & 89.00 & $\begin{array}{l}3 \mathrm{~d}^{3} 4 \mathrm{~s} 4 \mathrm{p} \\
{ }^{4} \mathrm{~F} \cdot{ }^{3} \mathrm{P} ;{ }^{4} \mathrm{D}\end{array}$ & 4.67 & $3 d^{3} 4 s 4 p{ }^{4} P .{ }^{3} P ;{ }^{4} D$ & -0.003 & \\
\hline & 89507.961 & 89576 & 58.41 & $3 \mathrm{~d}^{4} 4 \mathrm{p}^{3} \mathrm{P} ;{ }^{4} \mathrm{P}$ & 32.60 & $3 d^{4} 4 p^{3} \mathrm{P} ;{ }^{4} \mathrm{P}$ & 2.651 & \\
\hline & 90475.375 & 90549 & 35.99 & $3 d^{4} 4 p{ }^{3} P ;{ }^{4} D$ & 25.63 & $3 d^{4} 4 p^{3} F ;{ }^{4} D$ & 0.015 & \\
\hline & 92988.815 & 92943 & 98.10 & $3 d^{4} 5 p^{5} D ;{ }^{6} F$ & 1.11 & $3 d^{4} 5 p^{5} D ;{ }^{4} D$ & -0.660 & \\
\hline & & 93314 & 52.28 & $3 d^{4} 5 p^{5} D ;{ }^{6} D$ & 30.55 & $3 d^{4} 5 p^{5} D ;{ }^{4} P$ & 3.123 & \\
\hline & & 93675 & 64.65 & $\begin{array}{l}3 \mathrm{~d}^{3} 4 \mathrm{~s} 4 \mathrm{p} \\
{ }^{4} \mathrm{P} \cdot{ }^{3} \mathrm{P} ;{ }^{6} \mathrm{D}\end{array}$ & 31.97 & $3 d^{4} 5 p^{5} D ;{ }^{4} P$ & 3.099 & \\
\hline & 93800.484 & 93815 & 44.02 & $3 d^{4} 4 p^{3} F ;{ }^{4} D$ & 19.83 & $3 d^{4} 4 p^{3} P ;{ }^{4} D$ & 0.033 & \\
\hline & 93968.672 & 93931 & 46.54 & $3 d^{4} 5 p^{5} D ;{ }^{6} D$ & 34.56 & $3 d^{4} 5 p^{5} D ;{ }^{4} P$ & 3.065 & \\
\hline & 94624.703 & 94640 & 54.50 & $3 \mathrm{~d}^{4} 4 \mathrm{p}{ }^{3} \mathrm{P} ;{ }^{2} \mathrm{P}$ & 27.05 & $3 d^{4} 4 p^{3} \mathrm{P} ;{ }^{2} \mathrm{P}$ & 0.642 & \\
\hline & 94839.211 & 94764 & 82.72 & $3 d^{4} 5 p^{5} D ;{ }^{4} D$ & 4.46 & $3 \mathrm{~d}^{3} 4 \mathrm{~s} 4 \mathrm{p}{ }^{4} \mathrm{~F} .{ }^{1} \mathrm{P} ;{ }^{4} \mathrm{D}$ & 0.039 & \\
\hline & & 96711 & 51.34 & $3 d^{4} 4 p^{3} \mathrm{P} ;{ }^{2} \mathrm{~S}$ & 45.84 & $3 d^{4} 4 p^{3} p ;{ }^{2} S$ & 1.998 & \\
\hline & 97168.469 & 97369 & 78.91 & $\begin{array}{l}3 \mathrm{~d}^{3} 4 \mathrm{~s} 4 \mathrm{p} \\
{ }^{4} \mathrm{P} \cdot{ }^{3} \mathrm{P} ;{ }^{4} \mathrm{P}\end{array}$ & 8.30 & $3 d^{3} 4 s 4 p^{2}$ D. ${ }^{3} \mathrm{P} ;{ }^{4} \mathrm{P}$ & 2.660 & \\
\hline & & 100156 & 58.83 & $\begin{array}{l}3 d^{3} 4 s 4 p \\
{ }^{2} \mathrm{P}^{3} \mathrm{P} ;{ }^{4} \mathrm{P}\end{array}$ & 24.24 & $3 d^{3} 4 s 4 p^{2} D \cdot{ }^{3} P ;{ }^{4} \mathrm{P}$ & 2.623 & \\
\hline & & 100322 & 47.95 & $\begin{array}{l}3 d^{3} 4 s 4 p \\
{ }^{2} \mathrm{P} \cdot{ }^{3} \mathrm{P} ;{ }^{4} \mathrm{D}\end{array}$ & 32.52 & $3 d^{3} 4 s 4 p{ }^{4} P .{ }^{3} P ;{ }^{4} D$ & 0.068 & \\
\hline & & 101245 & 52.53 & $\begin{array}{l}3 \mathrm{~d}^{3} 4 \mathrm{~s} 4 \mathrm{p} \\
{ }^{4} \mathrm{P} \cdot{ }^{3} \mathrm{P} ;{ }^{2} \mathrm{P}\end{array}$ & 31.21 & $3 \mathrm{~d}^{3} 4 \mathrm{~s} 4 \mathrm{p}^{2} \mathrm{P} \cdot{ }^{3} \mathrm{P} ;{ }^{2} \mathrm{~S}$ & 1.086 & \\
\hline & & 101966 & 62.97 & $\begin{array}{l}3 \mathrm{~d}^{3} 4 \mathrm{~s} 4 \mathrm{p} \\
{ }^{2} \mathrm{P} \cdot{ }^{3} \mathrm{P} ;{ }^{2} \mathrm{~S}\end{array}$ & 28.29 & $3 \mathrm{~d}^{3} 4 \mathrm{~s} 4 \mathrm{p}{ }^{4} \mathrm{P} \cdot{ }^{3} \mathrm{P} ;{ }^{2} \mathrm{P}$ & 1.560 & \\
\hline & 102619.641 & 102797 & 54.42 & $\begin{array}{l}3 d^{3} 4 s 4 p \\
{ }^{4} \mathrm{P} \cdot{ }^{3} \mathrm{P} ;{ }^{4} \mathrm{D}\end{array}$ & 37.35 & $3 d^{3} 4 s 4 p^{2} P .{ }^{3} P ;{ }^{4} D$ & 0.013 & \\
\hline & & 103756 & 39.64 & $\begin{array}{l}3 d^{3} 4 s 4 p \\
{ }^{4} \mathrm{~F} .{ }^{1} \mathrm{P} ;{ }^{4} \mathrm{D}\end{array}$ & 34.28 & $3 d^{3} 4 s 4 p^{2}$ D. ${ }^{3} P ;{ }^{4} D$ & 0.009 & \\
\hline & 104439.570 & 104463 & 39.26 & $\begin{array}{l}3 d^{3} 4 s 4 p \\
{ }^{2} D .^{3} P ;{ }^{4} D\end{array}$ & 32.51 & $3 d^{3} 4 s 4 p^{4} F \cdot{ }^{1} \mathrm{P} ;{ }^{4} \mathrm{D}$ & 0.020 & \\
\hline & & 105121 & 45.07 & $\begin{array}{l}3 d^{3} 4 s 4 p \\
{ }^{2} \mathrm{D} \cdot{ }^{3} \mathrm{P} ;{ }^{2} \mathrm{P}\end{array}$ & 22.25 & $3 \mathrm{~d}^{3} 4 \mathrm{~s} 4 \mathrm{p}^{2} \mathrm{P} \cdot{ }^{3} \mathrm{P} ;{ }^{2} \mathrm{P}$ & 0.778 & \\
\hline & 105392.508 & 105364 & 52.97 & $3 d^{4} 4 f^{5} D ;{ }^{4} P$ & 23.77 & $3 d^{4} 4 f^{5} D ;{ }^{6} D$ & 2.145 & \\
\hline & & 105482 & 56.32 & $3 d^{4} 4 f^{5} D ;{ }^{6} F$ & 33.06 & $3 d^{4} 4 f^{5} D ;{ }^{4} P$ & 0.848 & \\
\hline & & 105524 & 58.02 & $3 d^{4} 4 f^{5} D ;{ }^{6} D$ & 26.65 & $3 d^{4} 4 f^{5} D ;{ }^{4} D$ & 1.888 & \\
\hline & & 105585 & 38.48 & $\begin{array}{l}3 d^{3} 4 s 4 p \\
2{ }^{3}{ }^{3} \mathrm{P} ;{ }^{4} \mathrm{P}\end{array}$ & 32.64 & $3 \mathrm{~d}^{3} 4 \mathrm{~s} 4 \mathrm{p}^{2} \mathrm{P} \cdot{ }^{3} \mathrm{P} ;{ }^{4} \mathrm{P}$ & 2.526 & \\
\hline & & 105650 & 58.48 & $3 d^{4} 4 f^{5} D ;{ }^{4} D$ & 19.59 & $3 d^{4} 4 f^{5} D ;{ }^{6} F$ & 0.457 & \\
\hline \multicolumn{9}{|c|}{ - } \\
\hline & 46905.137 & 46882 & 99.49 & $3 d^{4} 4 p^{5} \mathrm{D} ;{ }^{6} \mathrm{~F}$ & 0.24 & $3 d^{4} 5 p^{5} D ;{ }^{6} F$ & 1.067 & 1.124 \\
\hline & 48398.871 & 48337 & 96.69 & $3 d^{4} 4 p^{5} D ;{ }^{6} P$ & 2.71 & $3 d^{4} 4 p^{5} D ;{ }^{4} P$ & 2.383 & 2.382 \\
\hline & 49005.848 & 48920 & 73.32 & $3 d^{4} 4 p^{5} \mathrm{D} ;{ }^{4} \mathrm{P}$ & 21.01 & $3 d^{4} 4 p^{5} D ;{ }^{6} D$ & 1.783 & 1.802 \\
\hline & 49564.504 & 49510 & 78.38 & $3 d^{4} 4 p^{5} D ;{ }^{6} D$ & 20.85 & $3 d^{4} 4 p^{5} D ;{ }^{4} P$ & 1.840 & 1.824 \\
\hline & 51584.102 & 51804 & 95.62 & $3 d^{4} 4 p^{5} \mathrm{D} ;{ }^{4} \mathrm{~F}$ & 1.87 & $3 d^{4} 4 p^{3} F ;{ }^{4} F$ & 0.400 & 0.406 \\
\hline & 54499.492 & 54443 & 97.13 & $3 d^{4} 4 p^{5} D ;{ }^{4} D$ & 0.84 & $3 d^{4} 4 p^{3} p ;{ }^{4} D$ & 1.200 & 1.178 \\
\hline & 64061.680 & 64058 & 48.93 & $3 d^{4} 4 p^{3} p ;{ }^{4} D$ & 34.22 & $3 d^{4} 4 p^{3} p ;{ }^{4} D$ & 1.202 & 1.199 \\
\hline & 66354.758 & 66555 & 51.57 & $3 \mathrm{~d}^{4} 4 \mathrm{p}{ }^{3} \mathrm{P} ;{ }^{4} \mathrm{P}$ & 33.21 & $3 \mathrm{~d}^{4} 4 \mathrm{p}^{3} \mathrm{P} ;{ }^{4} \mathrm{P}$ & 1.676 & 1.671 \\
\hline & 66649.391 & 66747 & 25.19 & $3 d^{4} 4 p^{3} \mathrm{P} ;{ }^{2} \mathrm{P}$ & 21.91 & $3 d^{4} 4 p^{3} F ;{ }^{2} D$ & 1.078 & \\
\hline & 67070.445 & 66973 & 62.84 & $3 d^{4} 4 p^{3} F ;{ }^{4} F$ & 12.56 & $3 d^{4} 4 p^{3} F ;{ }^{4} F$ & 0.500 & \\
\hline & 67379.297 & 67382 & 31.57 & $3 \mathrm{~d}^{4} 4 \mathrm{p}^{3} \mathrm{P} ;{ }^{2} \mathrm{P}$ & 17.16 & $3 d^{4} 4 p{ }^{3} F ;{ }^{2} D$ & 1.035 & \\
\hline & 67870.211 & 68035 & 63.02 & $3 d^{4} 4 p^{3} F ;{ }^{4} D$ & 18.94 & $3 d^{4} 4 p^{3} F ;{ }^{4} D$ & 1.195 & \\
\hline
\end{tabular}


Table 5 (continued)

\begin{tabular}{|c|c|c|c|c|c|c|c|c|}
\hline$J$-value & \multirow{2}{*}{$\begin{array}{c}E_{o b s} \\
\left(\mathrm{~cm}^{-1}\right) \\
{[8,27]} \\
68305.555\end{array}$} & \multirow{2}{*}{$\begin{array}{c}\begin{array}{c}E_{\text {calc }} \\
\left(\mathrm{cm}^{-1}\right)\end{array} \\
68164\end{array}$} & \multicolumn{2}{|c|}{$\begin{array}{l}\text { Largest eigenvalue component } \\
\qquad(\%)\end{array}$} & \multicolumn{2}{|c|}{$\begin{array}{c}\text { Next largest eigenvalue component } \\
(\%)\end{array}$} & \multirow{2}{*}{$\begin{array}{c}\text { Calc. } g_{J} \\
1.974\end{array}$} & \multirow{2}{*}{$\begin{array}{c}\text { Obs. } g_{J} \\
{[5]} \\
1.978\end{array}$} \\
\hline & & & 52.07 & $3 d^{4} 4 p^{3} P ;{ }^{4} S$ & 42.86 & $3 \mathrm{~d}^{4} 4 \mathrm{p}^{3} \mathrm{P} ;{ }^{4} \mathrm{~S}$ & & \\
\hline & 69348.141 & 69473 & 35.24 & $3 d^{4} 4 p^{3} G ;{ }^{4} F$ & 27.20 & $3 d^{4} 4 p^{3} p ;{ }^{2} D$ & 0.637 & \\
\hline & 69638.594 & 69743 & 50.57 & $3 d^{4} 4 p^{3} G ;{ }^{4} F$ & 17.92 & $3 d^{4} 4 p{ }^{3} P ;{ }^{2} D$ & 0.571 & \\
\hline & 73411.883 & 73558 & 93.22 & $3 d^{4} 4 p^{3} D ;{ }^{4} D$ & 1.38 & $3 d^{4} 4 p^{3} D ;{ }^{4} F$ & 1.194 & \\
\hline & 74273.336 & 74293 & 85.27 & $3 d^{4} 4 p^{3} D ;{ }^{4} F$ & 10.95 & $3 d^{4} 4 p^{3} G ;{ }^{4} F$ & 0.422 & \\
\hline & 74717.555 & 74466 & 91.95 & $3 d^{4} 4 p^{3} D ;{ }^{4} P$ & 3.48 & $3 d^{4} 4 p^{3} \mathrm{P} ;{ }^{4} \mathrm{P}$ & 1.712 & \\
\hline & 74984.695 & 74905 & 56.44 & $3 d^{4} 4 p^{3} D ;{ }^{2} P$ & 28.91 & $3 \mathrm{~d}^{4} 4 \mathrm{p}{ }^{1} \mathrm{~S} ;{ }^{2} \mathrm{P}$ & 1.341 & \\
\hline & 77713.273 & 77640 & 37.11 & $3 d^{4} 4 p{ }^{1} s ;{ }^{2} P$ & 24.02 & $3 d^{4} 4 p^{3} D ;{ }^{2} P$ & 1.288 & \\
\hline & 78109.453 & 78086 & 75.78 & $3 d^{4} 4 p^{3} D ;{ }^{2} D$ & 6.18 & $3 d^{4} 4 p^{3} F ;{ }^{2} D$ & 0.843 & \\
\hline & & 78961 & 99.78 & $\begin{array}{l}3 d^{3} 4 s 4 p \\
{ }^{4} \mathrm{~F}^{3} \mathrm{P} ;{ }^{6} \mathrm{G}\end{array}$ & 0.08 & $3 d^{3} 4 s 4 p^{4} F \cdot{ }^{3} P ;{ }^{4} F$ & -0.002 & \\
\hline & 80288.023 & 80280 & 61.84 & $3 d^{4} 4 p^{1} D ;{ }^{2} D$ & 19.83 & $3 d^{4} 4 p^{1} D ;{ }^{2} D$ & 0.804 & \\
\hline & 81707.328 & 81687 & 92.04 & $\begin{array}{l}3 \mathrm{~d}^{3} 4 \mathrm{~s} 4 \mathrm{p} \\
{ }^{4} \mathrm{~F} \cdot{ }^{3} \mathrm{P} ;{ }^{6} \mathrm{~F}\end{array}$ & 6.91 & $3 d^{3} 4 s 4 p^{4} F \cdot{ }^{3} P ;{ }^{6} D$ & 1.125 & \\
\hline & 81824.070 & 81812 & 89.89 & $\begin{array}{l}3 \mathrm{~d}^{3} 4 \mathrm{~s} 4 \mathrm{p} \\
{ }^{4} \mathrm{~F} \cdot{ }^{3} \mathrm{P} ;{ }^{6} \mathrm{D}\end{array}$ & 7.26 & $3 d^{3} 4 s 4 p{ }^{4} \mathrm{~F} \cdot{ }^{3} \mathrm{P} ;{ }^{6} \mathrm{~F}$ & 1.809 & \\
\hline & 82919.914 & 82818 & 66.51 & $3 d^{4} 4 p^{1} D ;{ }^{2} P$ & 14.78 & $3 d^{4} 4 p^{1} \mathrm{D} ;{ }^{2} \mathrm{P}$ & 1.333 & \\
\hline & 85586.523 & 85443 & 88.24 & $\begin{array}{l}3 d^{3} 4 s 4 p \\
{ }^{4} F^{3} \mathrm{P} ;{ }^{4} \mathrm{D}\end{array}$ & 4.61 & $3 d^{3} 4 s 4 p^{4} \mathrm{P} \cdot{ }^{3} \mathrm{P} ;{ }^{4} \mathrm{D}$ & 1.198 & \\
\hline & 86919.008 & 86710 & 70.85 & $3 d^{4} 4 p^{1} F ;{ }^{2} D$ & 9.55 & $3 d^{4} 4 p^{3} P ;{ }^{2} D$ & 0.798 & \\
\hline & 87628.734 & 87802 & 67.02 & $\begin{array}{l}3 \mathrm{~d}^{3} 4 \mathrm{~s} 4 \mathrm{p} \\
{ }^{4} \mathrm{~F} \cdot{ }^{3} \mathrm{P} ;{ }^{4} \mathrm{~F}\end{array}$ & 15.19 & $3 d^{3} 4 s 4 p^{4} F \cdot{ }^{3} \mathrm{P} ;{ }^{2} \mathrm{D}$ & 0.476 & \\
\hline & 88604.250 & 88431 & 69.92 & $\begin{array}{l}3 d^{3} 4 s 4 p \\
{ }^{4} \mathrm{~F}^{3} \mathrm{P} ;{ }^{2} \mathrm{D}\end{array}$ & 12.75 & $3 d^{3} 4 s 4 p{ }^{4} F \cdot{ }^{3} \mathrm{P} ;{ }^{4} \mathrm{~F}$ & 0.726 & \\
\hline & 89422.328 & 89500 & 56.63 & $3 d^{4} 4 p^{3} \mathrm{P} ;{ }^{4} \mathrm{P}$ & 31.52 & $3 \mathrm{~d}^{4} 4 \mathrm{p}{ }^{3} \mathrm{P} ;{ }^{4} \mathrm{P}$ & 1.727 & \\
\hline & 90262.125 & 90005 & 66.11 & $3 d^{4} 4 p^{3} F ;{ }^{4} F$ & 16.27 & $3 d^{3} 4 s 4 p{ }^{4} F \cdot{ }^{3} P ;{ }^{4} F$ & 0.411 & \\
\hline & 90450.617 & 90508 & 34.42 & $3 \mathrm{~d}^{4} 4 \mathrm{p}^{3} \mathrm{P} ;{ }^{4} \mathrm{D}$ & 24.46 & $3 d^{4} 4 p^{3} F ;{ }^{4} D$ & 1.193 & \\
\hline & 91556.359 & 91585 & 31.27 & $3 d^{4} 4 p^{3} p ;{ }^{2} D$ & 23.25 & $3 d^{4} 4 p{ }^{3} p ;{ }^{2} D$ & 0.809 & \\
\hline & 92234.906 & 92323 & 50.02 & $3 d^{4} 4 p^{3} \mathrm{P} ;{ }^{4} \mathrm{~S}$ & 40.61 & $3 d^{4} 4 p^{3} P ;{ }^{4} S$ & 2.018 & \\
\hline & 92612.203 & 92395 & 68.75 & $\begin{array}{l}3 d^{3} 4 s 4 p \\
{ }^{4} \mathrm{P} \cdot{ }^{3} \mathrm{P} ;{ }^{6} \mathrm{P}\end{array}$ & 25.15 & $3 d^{4} 5 p^{5} \mathrm{D} ;{ }^{6} \mathrm{P}$ & 2.378 & \\
\hline & 93047.287 & 93017 & 98.05 & $3 d^{4} 5 p^{5} \mathrm{D} ;{ }^{6} \mathrm{~F}$ & 0.95 & $3 d^{4} 5 p^{5} D ;{ }^{4} D$ & 1.067 & \\
\hline & 93574.430 & 93441 & 56.09 & $3 d^{4} 5 p^{5} D ;{ }^{6} D$ & 19.77 & $3 d^{4} 5 p^{5} D ;{ }^{4} P$ & 1.850 & \\
\hline & 93740.241 & 93758 & 31.46 & $\begin{array}{l}3 \mathrm{~d}^{3} 4 \mathrm{~s} 4 \mathrm{p} \\
{ }^{4} \mathrm{P} .{ }^{3} \mathrm{P} ;{ }^{6} \mathrm{D}\end{array}$ & 22.92 & $3 d^{4} 5 p^{5} D ;{ }^{4} P$ & 1.800 & \\
\hline & 93769.992 & 93785 & 35.86 & $3 d^{4} 4 p^{3} F ;{ }^{4} D$ & 15.00 & $3 d^{4} 4 p^{3} P ;{ }^{4} D$ & 1.372 & \\
\hline & 94002.493 & 93876 & 41.40 & $3 d^{4} 5 p^{5} D ;{ }^{6} P$ & 27.35 & $3 d^{3} 4 s 4 p^{4} \mathrm{P} \cdot{ }^{3} \mathrm{P} ;{ }^{6} \mathrm{D}$ & 2.158 & \\
\hline & 94098.172 & 94045 & 45.93 & $3 d^{4} 5 p^{5} \mathrm{D} ;{ }^{4} \mathrm{P}$ & 26.20 & $3 d^{4} 5 p^{5} D ;{ }^{6} D$ & 1.870 & \\
\hline & 94255.977 & 94331 & 94.33 & $3 d^{4} 5 p^{5} D ;{ }^{4} F$ & 1.86 & $3 d^{4} 5 p^{5} D ;{ }^{4} D$ & 0.433 & \\
\hline & 94383.156 & 94425 & 57.55 & $3 d^{4} 4 p^{3} \mathrm{P} ;{ }^{2} \mathrm{P}$ & 28.69 & $3 d^{4} 4 p^{3} \mathrm{P} ;{ }^{2} \mathrm{P}$ & 1.332 & \\
\hline & 94932.942 & 94856 & 83.91 & $3 d^{4} 5 p^{5} D ;{ }^{4} D$ & 4.55 & $3 \mathrm{~d}^{3} 4 \mathrm{~s} 4 \mathrm{p}{ }^{4} \mathrm{~F} .{ }^{1} \mathrm{P} ;{ }^{4} \mathrm{D}$ & 1.192 & \\
\hline & 97294.047 & 97472 & 80.74 & $\begin{array}{l}3 \mathrm{~d}^{3} 4 \mathrm{~s} 4 \mathrm{p} \\
{ }^{4} \mathrm{P} .{ }^{3} \mathrm{P} ;{ }^{4} \mathrm{P}\end{array}$ & 7.18 & $3 d^{3} 4 s 4 p^{2}$ D. ${ }^{3} \mathrm{P} ;{ }^{4} \mathrm{P}$ & 1.734 & \\
\hline & 98314.984 & 98100 & 50.46 & $3 d^{4} 4 p^{3} \mathrm{~F} ;{ }^{2} \mathrm{D}$ & 18.84 & $3 d^{4} 4 p^{3} F ;{ }^{2} D$ & 0.800 & \\
\hline & 98578.555 & 98816 & 77.09 & $\begin{array}{l}3 \mathrm{~d}^{3} 4 \mathrm{~s} 4 \mathrm{p} \\
{ }^{2} \mathrm{G} \cdot{ }^{3} \mathrm{P} ;{ }^{4} \mathrm{~F}\end{array}$ & 12.35 & $3 \mathrm{~d}^{3} 4 \mathrm{~s} 4 \mathrm{p}^{2} \mathrm{D} \cdot{ }^{3} \mathrm{P} ;{ }^{4} \mathrm{~F}$ & 0.402 & \\
\hline & & 100299 & 54.31 & $\begin{array}{l}3 d^{3} 4 s 4 p \\
{ }^{2} \mathrm{P} \cdot{ }^{3} \mathrm{P} ;{ }^{4} \mathrm{P}\end{array}$ & 25.80 & $3 d^{3} 4 s 4 p^{2}$ D. ${ }^{3} P ;{ }^{4} \mathrm{P}$ & 1.724 & \\
\hline & 100691.820 & 100517 & 46.25 & $\begin{array}{l}3 d^{3} 4 s 4 p \\
2{ }^{3}{ }^{3} P ;{ }^{4} D\end{array}$ & 32.53 & $3 d^{3} 4 s 4 p{ }^{4} P .{ }^{3} P ;{ }^{4} D$ & 1.191 & \\
\hline & 101157.773 & 101417 & 77.12 & $\begin{array}{l}3 \mathrm{~d}^{3} 4 \mathrm{~s} 4 \mathrm{p} \\
{ }^{4} \mathrm{P} \cdot{ }^{3} \mathrm{P} ;{ }^{2} \mathrm{P}\end{array}$ & 4.19 & $3 d^{3} 4 s 4 p^{2} P \cdot{ }^{3} P ;{ }^{4} D$ & 1.310 & \\
\hline & 101986.992 & 102009 & 42.45 & $\begin{array}{l}3 \mathrm{~d}^{3} 4 \mathrm{~s} 4 \mathrm{p} \\
{ }^{2} \mathrm{D} \cdot{ }^{3} \mathrm{P} ;{ }^{4} \mathrm{~F}\end{array}$ & 17.84 & $3 \mathrm{~d}^{3} 4 \mathrm{~s} 4 \mathrm{p}^{2} \mathrm{G} \cdot{ }^{3} \mathrm{P} ;{ }^{4} \mathrm{~F}$ & 0.478 & \\
\hline & 102602.312 & 102485 & 84.44 & $\begin{array}{l}3 \mathrm{~d}^{3} 4 \mathrm{~s} 4 \mathrm{p} \\
{ }^{2} \mathrm{P} \cdot{ }^{3} \mathrm{P} ;{ }^{4} \mathrm{~S}\end{array}$ & 9.86 & $3 d^{4} 4 p{ }^{4} P .{ }^{3} P ;{ }^{4} S$ & 1.986 & \\
\hline & 102684.391 & 102787 & 48.06 & $\begin{array}{l}3 \mathrm{~d}^{3} 4 \mathrm{~s} 4 \mathrm{p} \\
{ }^{4} \mathrm{P} \cdot{ }^{3} \mathrm{P} ;{ }^{4} \mathrm{D}\end{array}$ & 35.99 & $3 d^{3} 4 s 4 p^{2} P .{ }^{3} P ;{ }^{4} D$ & 1.176 & \\
\hline & & 103184 & 43.21 & $\begin{array}{l}3 d^{3} 4 s 4 p \\
{ }^{2} \mathrm{P} \cdot{ }^{3} \mathrm{P} ;{ }^{2} \mathrm{D}\end{array}$ & 21.36 & $3 d^{3} 4 s 4 p^{2}$ D. ${ }^{3} P ;{ }^{2} D$ & 0.798 & \\
\hline & 104274.570 & 103907 & 37.61 & $\begin{array}{l}3 d^{3} 4 s 4 p \\
{ }^{2} D \cdot{ }^{3} P ;{ }^{4} D\end{array}$ & 35.39 & $3 \mathrm{~d}^{3} 4 \mathrm{~s} 4 \mathrm{p}^{4} \mathrm{~F} \cdot{ }^{1} \mathrm{P} ;{ }^{4} \mathrm{D}$ & 1.203 & \\
\hline & 104616.250 & 104559 & 32.41 & $\begin{array}{l}3 d^{3} 4 s 4 p \\
{ }^{4} \mathrm{~F} .{ }^{1} \mathrm{P} ;{ }^{4} \mathrm{D}\end{array}$ & 30.33 & $3 d^{3} 4 s 4 p^{2}$ D. ${ }^{3} P ;{ }^{4} D$ & 1.220 & \\
\hline & & 104751 & 43.06 & $\begin{array}{l}3 \mathrm{~d}^{3} 4 \mathrm{~s} 4 \mathrm{p} \\
{ }^{2} \mathrm{D} \cdot{ }^{3} \mathrm{P} ;{ }^{2} \mathrm{P}\end{array}$ & 17.77 & $3 d^{3} 4 s 4 p^{2}$ D. ${ }^{3} \mathrm{P} ;{ }^{2} \mathrm{P}$ & 1.335 & \\
\hline & & 104988 & 45.78 & $\begin{array}{l}3 d^{3} 4 s 4 p \\
{ }^{4} \mathrm{~F} \cdot{ }^{1} \mathrm{P} ;{ }^{4} \mathrm{~F}\end{array}$ & 35.05 & $3 d^{4} 4 f^{5} D ;{ }^{4} F$ & 0.420 & \\
\hline & 105283.430 & 105256 & 62.24 & $3 d^{4} 4 f^{5} D ;{ }^{4} P$ & 13.40 & $3 d^{4} 4 f^{5} D ;{ }^{6} D$ & 1.657 & \\
\hline & & 105386 & 29.87 & $3 d^{4} 4 f^{5} D ;{ }^{6} D$ & 25.81 & $3 d^{4} 4 f^{5} D ;{ }^{6} F$ & 1.404 & \\
\hline & & 105447 & 49.77 & $3 d^{4} 4 f^{5} D ;{ }^{6} G$ & 19.88 & $3 d^{4} 4 f^{5} D ;{ }^{6} \mathrm{P}$ & 0.922 & \\
\hline
\end{tabular}


Table 5 (continued)

\begin{tabular}{|c|c|c|c|c|c|c|c|c|}
\hline \multirow[t]{2}{*}{$J$-value } & \multirow{2}{*}{$\begin{array}{c}E_{o b s} \\
\left(\mathrm{~cm}^{-1}\right) \\
{[8,27]}\end{array}$} & \multirow{2}{*}{$\begin{array}{c}\begin{array}{c}E_{\text {calc }} \\
\left(\mathrm{cm}^{-1}\right)\end{array} \\
105480\end{array}$} & \multicolumn{2}{|c|}{$\begin{array}{l}\text { Largest eigenvalue component } \\
\qquad(\%)\end{array}$} & \multicolumn{2}{|c|}{$\begin{array}{c}\text { Next largest eigenvalue component } \\
(\%)\end{array}$} & \multirow{2}{*}{$\begin{array}{r}\text { Calc. } g_{J} \\
1.716\end{array}$} & \multirow{2}{*}{$\begin{array}{c}\text { Obs. } g_{J} \\
{[5]}\end{array}$} \\
\hline & & & 35.30 & $\begin{array}{l}3 d^{3} 4 s 4 p \\
{ }^{2} \mathrm{D} .{ }^{3} \mathrm{P} ;{ }^{4} \mathrm{P}\end{array}$ & 30.83 & $3 \mathrm{~d}^{3} 4 \mathrm{~s} 4 \mathrm{p}^{2} \mathrm{P} \cdot{ }^{3} \mathrm{P} ;{ }^{4} \mathrm{P}$ & & \\
\hline & & 105544 & 31.72 & $3 d^{4} 4 f^{5} D ;{ }^{6} G$ & 30.42 & $3 d^{4} 4 f^{5} D ;{ }^{6} F$ & 1.086 & \\
\hline & & 105629 & 39.25 & $3 d^{4} 4 f^{5} D ;{ }^{4} D$ & 33.71 & $3 d^{4} 4 f^{5} D ;{ }^{6} P$ & 1.698 & \\
\hline & & 105676 & 30.07 & $3 d^{4} 4 f^{5} D ;{ }^{6} F$ & 28.22 & $3 d^{4} 4 f^{5} D ;{ }^{6} D$ & 1.437 & \\
\hline & & 105946 & 60.12 & $3 d^{4} 4 f^{5} D ;{ }^{4} F$ & 23.78 & $3 d^{3} 4 s 4 p^{4} F \cdot{ }^{1} \mathrm{P} ;{ }^{4} \mathrm{~F}$ & 0.463 & \\
\hline & 106726.086 & 106544 & 81.23 & $\begin{array}{l}3 \mathrm{~d}^{3} 4 \mathrm{~s} 4 \mathrm{p} \\
{ }^{4} \mathrm{P} .{ }^{3} \mathrm{P} ;{ }^{4} \mathrm{~S}\end{array}$ & 10.67 & $3 d^{3} 4 s 4 p^{2} P \cdot{ }^{3} P ;{ }^{4} S$ & 1.979 & \\
\hline & 107212.344 & 106852 & 85.12 & $\begin{array}{l}3 d^{3} 4 s 4 p \\
{ }^{4} \mathrm{P} \cdot{ }^{3} \mathrm{P} ;{ }^{2} \mathrm{D}\end{array}$ & 4.89 & $3 d^{3} 4 s 4 p^{4} F \cdot{ }^{3} P ;{ }^{2} D$ & 0.812 & \\
\hline & & 109178 & 71.68 & $\begin{array}{l}3 d^{3} 4 s 4 p \\
{ }^{2} \mathrm{P} \cdot{ }^{3} \mathrm{P} ;{ }^{2} \mathrm{P}\end{array}$ & 14.16 & $3 d^{3} 4 s 4 p^{2}$ D. ${ }^{3} \mathrm{P} ;{ }^{2} \mathrm{P}$ & 1.328 & \\
\hline & & 109515 & 94.76 & $3 d^{4} 6 p^{5} \mathrm{D} ;{ }^{6} \mathrm{~F}$ & 1.85 & $3 d^{4} 6 p^{5} D ;{ }^{4} P$ & 1.086 & \\
\hline & 109661.352 & 109563 & 54.47 & $3 \mathrm{~d}^{4} 6 \mathrm{p}^{5} \mathrm{D} ;{ }^{4} \mathrm{P}$ & 24.54 & $3 d^{4} 6 p^{5} D ;{ }^{6} D$ & 1.835 & \\
\hline & & 109880 & 73.59 & $3 \mathrm{~d}^{4} 6 \mathrm{p}^{5} \mathrm{D} ;{ }^{6} \mathrm{P}$ & 25.12 & $3 d^{4} 6 p^{5} D ;{ }^{6} D$ & 2.259 & \\
\hline & & 110039 & 49.36 & $3 d^{4} 6 p^{5} D ;{ }^{6} D$ & 32.09 & $3 d^{4} 6 p^{5} D ;{ }^{4} P$ & 1.872 & \\
\hline & 109914.492 & 110147 & 42.42 & $\begin{array}{l}3 \mathrm{~d}^{3} 4 \mathrm{~s} 4 \mathrm{p} \\
{ }^{2} \mathrm{D} \cdot{ }^{3} \mathrm{P} ;{ }^{2} \mathrm{D}\end{array}$ & 37.87 & $3 d^{3} 4 s 4 p^{2} \mathrm{P} \cdot{ }^{3} \mathrm{P} ;{ }^{2} \mathrm{D}$ & 0.803 & \\
\hline & & 110316 & 90.13 & $3 d^{4} 6 p^{5} D ;{ }^{4} F$ & 2.39 & $3 d^{3} 4 s 4 p{ }^{4} F \cdot{ }^{1} \mathrm{P} ;{ }^{4} \mathrm{~F}$ & 0.440 & \\
\hline & & 110435 & 48.04 & $3 d^{4} 5 p{ }^{3} p ;{ }^{4} D$ & 32.95 & $3 d^{4} 5 p^{3} P ;{ }^{4} D$ & 1.205 & \\
\hline & & 110895 & 22.35 & $3 d^{4} 5 p^{3} \mathrm{P} ;{ }^{4} \mathrm{P}$ & 15.70 & $3 d^{4} 5 p^{3} P ;{ }^{2} \mathrm{P}$ & 1.559 & \\
\hline & 110931.633 & 111052 & 83.18 & $3 d^{4} 6 p^{5} D ;{ }^{4} D$ & 4.91 & $3 \mathrm{~d}^{3} 4 \mathrm{~s} 4 \mathrm{p}{ }^{4} \mathrm{~F} \cdot{ }^{1} \mathrm{P} ;{ }^{4} \mathrm{D}$ & 1.221 & \\
\hline & & 111231 & 27.52 & $3 \mathrm{~d}^{4} 5 \mathrm{p}^{3} \mathrm{P} ;{ }^{4} \mathrm{P}$ & 20.50 & $3 d^{4} 5 p^{3} p ;{ }^{2} \mathrm{P}$ & 1.493 & \\
\hline & & 111567 & 32.53 & $3 d^{4} 5 p^{3} p ;{ }^{4} S$ & 23.47 & $3 d^{4} 5 p{ }^{3} p ;{ }^{2} D$ & 1.500 & \\
\hline & & 111731 & 32.78 & $3 d^{4} 5 p^{3} F ;{ }^{4} F$ & 27.88 & $3 d^{4} 5 p^{3} F ;{ }^{2} D$ & 0.719 & \\
\hline & & 111811 & 23.33 & $3 d^{4} 4 p^{1} D ;{ }^{2} P$ & 17.24 & $3 d^{4} 5 p^{3} p ;{ }^{2} D$ & 1.191 & \\
\hline & & 111980 & 42.68 & $3 \mathrm{~d}^{4} 4 \mathrm{p}{ }^{1} \mathrm{D} ;{ }^{2} \mathrm{P}$ & 11.86 & $3 d^{4} 4 p^{1} D ;{ }^{2} P$ & 1.311 & \\
\hline & & 112158 & 43.03 & $3 d^{4} 5 p^{3} F ;{ }^{4} D$ & 20.74 & $3 d^{4} 5 p^{3} F ;{ }^{4} F$ & 0.949 & \\
\hline & & 112229 & 36.20 & $3 d^{4} 5 p^{3} F ;{ }^{2} D$ & 18.95 & $3 d^{4} 5 p^{3} F ;{ }^{4} D$ & 0.826 & \\
\hline & & 114106 & 55.25 & $3 d^{4} 5 p^{3} G ;{ }^{4} F$ & 39.84 & $3 d^{3} 4 s 4 p^{2} F \cdot{ }^{3} \mathrm{P} ;{ }^{4} \mathrm{~F}$ & 0.404 & \\
\hline & & 115080 & 52.69 & $\begin{array}{l}3 \mathrm{~d}^{3} 4 \mathrm{~s} 4 \mathrm{p} \\
{ }^{2} \mathrm{~F} \cdot{ }^{3} \mathrm{P} ;{ }^{4} \mathrm{~F}\end{array}$ & 33.60 & $3 d^{4} 5 p^{3} G ;{ }^{4} F$ & 0.416 & \\
\hline & & 115176 & 58.22 & $3 d^{4} 5 f^{5} D ;{ }^{4} P$ & 20.25 & $3 \mathrm{~d}^{3} 4 \mathrm{~s} 4 \mathrm{p}^{4} \mathrm{P} .{ }^{1} \mathrm{P} ;{ }^{4} \mathrm{P}$ & 1.642 & \\
\hline & & 115381 & 42.68 & $3 d^{4} 5 f^{5} D ;{ }^{6} G$ & 34.01 & $3 d^{4} 4 p^{5} \mathrm{D} ;{ }^{6} \mathrm{~F}$ & 0.736 & \\
\hline & 115430.875 & 115456 & 40.96 & $3 d^{4} 5 f^{5} D ;{ }^{6} D$ & 19.62 & $3 d^{4} 4 p^{5} D ;{ }^{6} G$ & 1.249 & \\
\hline & & 115505 & 46.29 & $3 d^{4} 5 f^{5} D ;{ }^{4} D$ & 30.34 & $3 d^{4} 4 p^{5} D ;{ }^{6} G$ & 0.831 & \\
\hline & 115605.898 & 115599 & 33.31 & $3 d^{4} 5 f^{5} D ;{ }^{6} F$ & 18.25 & $3 d^{4} 5 f^{5} D ;{ }^{6} P$ & 1.259 & \\
\hline & & 115610 & 58.66 & $3 d^{4} 4 p^{1} D ;{ }^{2} D$ & 20.26 & $3 d^{4} 4 p^{1} D ;{ }^{2} D$ & 0.888 & \\
\hline & & 115721 & 54.40 & $3 d^{4} 5 f^{5} D ;{ }^{4} F$ & 21.75 & $3 d^{4} 4 p^{5} D ;{ }^{4} D$ & 0.742 & \\
\hline & 115747.539 & 115750 & 61.71 & $3 d^{4} 5 f^{5} D ;{ }^{6} P$ & 28.72 & $3 d^{4} 4 p^{5} D ;{ }^{6} D$ & 2.112 & \\
\hline & 115966.555 & 116200 & 55.52 & $\begin{array}{l}3 d^{3} 4 s{ }^{4 p} \\
{ }^{4} \mathrm{P} .{ }^{1} \mathrm{P} ;{ }^{4} \mathrm{P}\end{array}$ & 32.24 & $3 d^{4} 5 f^{5} D ;{ }^{4} P$ & 1.727 & \\
\hline & & 117167 & 48.18 & $\begin{array}{l}3 \mathrm{~d}^{3} 4 \mathrm{~s} 4 \mathrm{p} \\
{ }^{2} \mathrm{~F}^{3} \mathrm{P} ;{ }^{4} \mathrm{D}\end{array}$ & 36.50 & $3 \mathrm{~d}^{3} 4 \mathrm{~s} 4 \mathrm{p}{ }^{4} \mathrm{P} \cdot{ }^{1} \mathrm{P} ;{ }^{4} \mathrm{D}$ & 1.201 & \\
\hline & 118622.562 & 118792 & 44.64 & $\begin{array}{l}3 d^{3} 4 s 4 p \\
{ }^{4} \mathrm{P} .{ }^{1} \mathrm{P} ;{ }^{4} \mathrm{D}\end{array}$ & 44.36 & $3 \mathrm{~d}^{3} 4 \mathrm{~s} 4 \mathrm{p}^{2} \mathrm{~F} \cdot{ }^{3} \mathrm{P} ;{ }^{4} \mathrm{D}$ & 1.199 & \\
\hline & & 120098 & 50.78 & $\begin{array}{l}3 \mathrm{~d}^{3} 4 \mathrm{~s} 4 \mathrm{p} \\
{ }^{2} \mathrm{~F}^{3} \mathrm{P} ;{ }^{2} \mathrm{D}\end{array}$ & 14.72 & $3 d^{4} 5 p^{3} D ;{ }^{2} D$ & 0.795 & \\
\hline & & 120332 & 73.28 & $3 d^{4} 5 p^{3} D ;{ }^{4} F$ & 11.76 & $3 d^{4} 5 p^{3} D ;{ }^{4} D$ & 0.573 & \\
\hline & & 120470 & 42.18 & $3 d^{4} 5 p^{3} D ;{ }^{4} D$ & 19.45 & $3 d^{4} 5 p^{3} D ;{ }^{2} P$ & 1.132 & \\
\hline & & 120632 & 32.37 & $3 d^{4} 5 p^{3} D ;{ }^{2} D$ & 13.39 & $3 \mathrm{~d}^{3} 4 \mathrm{~s} 4 \mathrm{p}^{2} \mathrm{~F} \cdot{ }^{3} \mathrm{P} ;{ }^{2} \mathrm{D}$ & 0.905 & \\
\hline & & 120834 & 50.01 & $3 d^{4} 5 p^{3} D ;{ }^{2} P$ & 24.39 & $3 d^{4} 5 p^{3} D ;{ }^{4} D$ & 1.268 & \\
\hline & & 120901 & 24.09 & $\begin{array}{l}3 \mathrm{~d}^{3} 4 \mathrm{~s} 4 \mathrm{p} \\
{ }^{2} \mathrm{P} .{ }^{1} \mathrm{P} ;{ }^{2} \mathrm{P}\end{array}$ & 16.83 & $3 \mathrm{~d}^{3} 4 \mathrm{~s} 4 \mathrm{p}^{2}$ D. ${ }^{1} \mathrm{P} ;{ }^{2} \mathrm{P}$ & 1.179 & \\
\hline & & 120944 & 84.33 & $3 d^{4} 4 f^{3} \mathrm{H} ;{ }^{4} \mathrm{~F}$ & 3.80 & $3 d^{4} 4 f^{3} H ;{ }^{2} D$ & 0.499 & \\
\hline \multicolumn{9}{|l|}{2.5} \\
\hline & 47040.273 & 47001 & 99.51 & $3 d^{4} 4 p^{5} \mathrm{D} ;{ }^{6} \mathrm{~F}$ & 0.24 & $3 d^{4} 5 p^{5} \mathrm{D} ;{ }^{6} \mathrm{~F}$ & 1.315 & 1.314 \\
\hline & 48491.059 & 48423 & 97.41 & $3 \mathrm{~d}^{4} 4 \mathrm{p}^{5} \mathrm{D} ;{ }^{6} \mathrm{P}$ & 2.08 & $3 d^{4} 4 p^{5} D ;{ }^{4} P$ & 1.881 & 1.875 \\
\hline & 49351.734 & 49267 & 66.85 & $3 d^{4} 4 p^{5} D ;{ }^{6} D$ & 31.86 & $3 d^{4} 4 p^{5} D ;{ }^{4} P$ & 1.640 & 1.628 \\
\hline & 49706.262 & 49603 & 63.07 & $3 d^{4} 4 p^{5} \mathrm{D} ;{ }^{4} \mathrm{P}$ & 32.55 & $3 d^{4} 4 p^{5} D ;{ }^{6} D$ & 1.626 & \\
\hline & 51669.406 & 51876 & 95.51 & $3 d^{4} 4 p^{5} D ;{ }^{4} F$ & 1.84 & $3 d^{4} 4 p^{3} F ;{ }^{4} F$ & 1.030 & 1.025 \\
\hline & 54625.594 & 54559 & 97.13 & $3 d^{4} 4 p^{5} D ;{ }^{4} D$ & 0.83 & $3 d^{4} 4 p^{3} D ;{ }^{4} D$ & 1.372 & 1.376 \\
\hline & 64448.750 & 64387 & 47.68 & $3 d^{4} 4 p^{3} P ;{ }^{4} D$ & 33.40 & $3 d^{4} 4 p^{3} p ;{ }^{4} D$ & 1.372 & 1.38 \\
\hline & 65156.492 & 65273 & 51.44 & $3 d^{4} 4 p^{3} F ;{ }^{4} G$ & 21.25 & $3 d^{4} 4 p^{3} F ;{ }^{4} G$ & 0.573 & 0.593 \\
\hline & 66726.781 & 66895 & 44.25 & $3 d^{4} 4 p{ }^{3} P ;{ }^{4} \mathrm{P}$ & 28.34 & $3 d^{4} 4 p^{3} P ;{ }^{4} P$ & 1.505 & 1.502 \\
\hline & 67012.078 & 67104 & 30.66 & $3 d^{4} 4 p^{3} F ;{ }^{2} D$ & 16.39 & $3 d^{4} 4 p^{3} F ;{ }^{4} F$ & 1.238 & \\
\hline & 67344.008 & 67317 & 74.02 & $3 d^{4} 4 p^{3} H ;{ }^{4} G$ & 15.30 & $3 d^{4} 4 p^{3} F ;{ }^{4} G$ & 0.584 & \\
\hline & 67387.094 & 67397 & 60.33 & $3 d^{4} 4 p^{3} F ;{ }^{4} F$ & 11.91 & $3 \mathrm{~d}^{4} 4 \mathrm{p}^{3} \mathrm{~F} ;{ }^{4} \mathrm{~F}$ & 1.073 & \\
\hline & 67867.789 & 68034 & 57.32 & $3 d^{4} 4 p{ }^{3} \mathrm{~F} ;{ }^{4} \mathrm{D}$ & 16.86 & $3 d^{4} 4 p^{3} F ;{ }^{4} D$ & 1.337 & \\
\hline & 68583.305 & 68542 & 32.72 & $3 d^{4} 4 p^{3} F ;{ }^{2} F$ & 30.46 & $3 d^{4} 4 p^{3} G ;{ }^{2} F$ & 0.881 & \\
\hline & 69954.055 & 69604 & 43.26 & $3 d^{4} 4 p^{3} \mathrm{P} ;{ }^{2} \mathrm{D}$ & 30.51 & $3 d^{4} 4 p^{3} P ;{ }^{2} D$ & 1.186 & \\
\hline
\end{tabular}


Table 5 (continued)

\begin{tabular}{|c|c|c|c|c|c|c|c|c|}
\hline$J$-value & \multirow{2}{*}{$\begin{array}{c}E_{o b s} \\
\left(\mathrm{~cm}^{-1}\right) \\
{[8,27]} \\
69477.898\end{array}$} & \multirow{2}{*}{$\begin{array}{c}\begin{array}{c}E_{\text {calc }} \\
\left(\mathrm{cm}^{-1}\right)\end{array} \\
69998\end{array}$} & \multicolumn{2}{|c|}{$\begin{array}{c}\text { Largest eigenvalue component } \\
(\%)\end{array}$} & \multicolumn{2}{|c|}{$\begin{array}{l}\text { Next largest eigenvalue component } \\
(\%)\end{array}$} & \multirow{2}{*}{$\begin{array}{r}\text { Calc. } g_{J} \\
1.023\end{array}$} & $\begin{array}{c}\text { Obs. } g_{J} \\
{[5]}\end{array}$ \\
\hline & & & 76.94 & $3 d^{4} 4 p^{3} G ;{ }^{4} F$ & 9.44 & $3 d^{4} 4 p^{3} D ;{ }^{4} F$ & & \\
\hline & 70316.820 & 70219 & 71.08 & $3 d^{4} 4 p^{3} G ;{ }^{4} G$ & 12.58 & $3 d^{4} 4 p^{3} \mathrm{H} ;{ }^{4} \mathrm{G}$ & 0.600 & \\
\hline & 70584.391 & 70448 & 45.01 & $3 d^{4} 4 p^{3} G ;{ }^{2} F$ & 35.96 & $3 d^{4} 4 p^{3} F ;{ }^{2} F$ & 0.856 & \\
\hline & 73436.125 & 73577 & 91.46 & $3 d^{4} 4 p^{3} D ;{ }^{4} D$ & 2.54 & $3 d^{4} 4 p^{3} D ;{ }^{4} F$ & 1.365 & \\
\hline & 74318.844 & 74248 & 84.13 & $3 d^{4} 4 p^{3} D ;{ }^{4} P$ & 6.59 & $3 d^{4} 4 p^{3} D ;{ }^{4} F$ & 1.529 & \\
\hline & 74436.117 & 74294 & 35.72 & $3 d^{4} 4 p^{1} G ;{ }^{2} F$ & 17.07 & $3 d^{4} 4 p^{1} G ;{ }^{2} F$ & 0.961 & \\
\hline & 74483.906 & 74383 & 61.59 & $3 d^{4} 4 p^{3} D ;{ }^{4} F$ & 14.51 & $3 d^{4} 4 p{ }^{1} G ;{ }^{2} F$ & 1.003 & \\
\hline & 76987.672 & 76938 & 72.72 & $3 d^{4} 4 p{ }^{3} D ;{ }^{2} F$ & 7.82 & $3 d^{4} 4 p^{3} G ;{ }^{2} F$ & 0.858 & \\
\hline & 77935.203 & 77905 & 77.02 & $3 d^{4} 4 p^{3} D ;{ }^{2} D$ & 9.53 & $3 d^{4} 4 p^{1} D ;{ }^{2} D$ & 1.201 & \\
\hline & & 79115 & 99.82 & $\begin{array}{l}3 \mathrm{~d}^{3} 4 \mathrm{~s} 4 \mathrm{p} \\
{ }^{4} \mathrm{~F} \cdot{ }^{3} \mathrm{P} ;{ }^{6} \mathrm{G}\end{array}$ & 0.06 & $3 d^{3} 4 s$ p ${ }^{4} F \cdot{ }^{3} \mathrm{P} ;{ }^{4} \mathrm{~F}$ & 0.857 & \\
\hline & 80420.164 & 80431 & 58.30 & $3 d^{4} 4 p^{1} D ;{ }^{2} D$ & 18.42 & $3 d^{4} 4 p{ }^{1} D ;{ }^{2} D$ & 1.195 & \\
\hline & 81232.883 & 81189 & 68.03 & $3 d^{4} 4 p{ }^{1} D ;{ }^{2} F$ & 17.83 & $3 d^{4} 4 p^{1} \mathrm{D} ;{ }^{2} \mathrm{~F}$ & 0.862 & \\
\hline & 81815.883 & 81830 & 82.80 & $\begin{array}{l}3 d^{3} 4 s 4 p \\
{ }^{4} \mathrm{~F} \cdot{ }^{3} \mathrm{P} ;{ }^{6} \mathrm{D}\end{array}$ & 14.47 & $3 d^{3} 4 s 4 p{ }^{4} F \cdot{ }^{3} \mathrm{P} ;{ }^{6} \mathrm{~F}$ & 1.608 & \\
\hline & 81961.867 & 81943 & 84.98 & $\begin{array}{l}3 \mathrm{~d}^{3} 4 \mathrm{~s} 4 \mathrm{p} \\
{ }^{4} \mathrm{~F} \cdot{ }^{3} \mathrm{P} ;{ }^{6} \mathrm{~F}\end{array}$ & 13.85 & $3 d^{3} 4 s 4 p{ }^{4} F \cdot{ }^{3} \mathrm{P} ;{ }^{6} \mathrm{D}$ & 1.364 & \\
\hline & 84604.641 & 84394 & 87.93 & $3 d^{4} 4 p^{1} F ;{ }^{2} F$ & 3.20 & $3 d^{4} 4 p^{3} D ;{ }^{2} F$ & 0.859 & \\
\hline & 85778.656 & 85652 & 88.04 & $\begin{array}{l}3 \mathrm{~d}^{3} 4 \mathrm{~s} 4 \mathrm{p} \\
{ }^{4} \mathrm{~F} \cdot{ }^{3} \mathrm{P} ;{ }^{4} \mathrm{D}\end{array}$ & 4.58 & $3 \mathrm{~d}^{3} 4 \mathrm{~s} 4 \mathrm{p}{ }^{4} \mathrm{P} .{ }^{3} \mathrm{P} ;{ }^{4} \mathrm{D}$ & 1.371 & \\
\hline & 86507.328 & 86353 & 70.78 & $3 d^{4} 4 p{ }^{1} F ;{ }^{2} D$ & 10.06 & $3 d^{4} 4 p^{3} P ;{ }^{2} D$ & 1.199 & \\
\hline & 86566.461 & 86669 & 93.63 & $\begin{array}{l}3 \mathrm{~d}^{3} 4 \mathrm{~s} 4 \mathrm{p} \\
{ }^{4} \mathrm{~F} .{ }^{3} \mathrm{P} ;{ }^{4} \mathrm{G}\end{array}$ & 3.77 & $3 \mathrm{~d}^{3} 4 \mathrm{~s} 4 \mathrm{p}^{2} \mathrm{G} \cdot{ }^{3} \mathrm{P} ;{ }^{4} \mathrm{G}$ & 0.572 & \\
\hline & 87766.594 & 88023 & 76.52 & $\begin{array}{l}3 d^{3} 4 s 4 p \\
{ }^{4} \mathrm{~F} \cdot{ }^{3} \mathrm{P} ;{ }^{4} \mathrm{~F}\end{array}$ & 14.84 & $3 d^{4} 4 p^{3} F ;{ }^{4} F$ & 1.034 & \\
\hline & 89164.516 & 88910 & 82.10 & $\begin{array}{l}3 \mathrm{~d}^{3} 4 \mathrm{~s} 4 \mathrm{p} \\
{ }^{4} \mathrm{~F} \cdot{ }^{3} \mathrm{P} ;{ }^{2} \mathrm{D}\end{array}$ & 5.55 & $3 \mathrm{~d}^{3} 4 \mathrm{~s} 4 \mathrm{p}{ }^{4} \mathrm{P} .{ }^{3} \mathrm{P} ;{ }^{2} \mathrm{D}$ & 1.195 & \\
\hline & 89453.031 & 89518 & 56.85 & $3 d^{4} 4 p{ }^{3} p ;{ }^{4} \mathrm{P}$ & 31.61 & $3 d^{4} 4 p^{3} p ;{ }^{4} \mathrm{P}$ & 1.593 & \\
\hline & 90258.195 & 90035 & 32.25 & $3 d^{4} 4 p^{3} P ;{ }^{4} D$ & 23.01 & $3 d^{4} 4 p^{3} F ;{ }^{4} D$ & 1.356 & \\
\hline & 90441.766 & 90447 & 62.75 & $3 d^{4} 4 p{ }^{3} F ;{ }^{4} F$ & 17.68 & $3 d^{3} 4 s$ p ${ }^{4} \mathrm{~F} \cdot{ }^{3} \mathrm{P} ;{ }^{4} \mathrm{~F}$ & 1.041 & \\
\hline & 90706.805 & 91017 & 36.96 & $3 d^{4} 4 p{ }^{3} F ;{ }^{2} F$ & 21.60 & $3 d^{4} 4 p{ }^{3} F ;{ }^{4} G$ & 0.774 & \\
\hline & 91078.711 & 91269 & 50.75 & $3 d^{4} 4 p^{3} F ;{ }^{4} G$ & 16.95 & $3 d^{4} 4 p{ }^{3} F ;{ }^{4} G$ & 0.660 & \\
\hline & 91426.047 & 91441 & 31.30 & $3 d^{4} 4 p^{3} P ;{ }^{2} D$ & 23.62 & $3 d^{4} 4 p^{3} P ;{ }^{2} D$ & 1.208 & \\
\hline & 92417.891 & 92542 & 70.50 & $\begin{array}{l}3 \mathrm{~d}^{3} 4 \mathrm{~s} 4 \mathrm{p} \\
{ }^{4} \mathrm{P} .{ }^{3} \mathrm{P} ;{ }^{6} \mathrm{P}\end{array}$ & 28.60 & $3 d^{4} 5 p^{5} \mathrm{D} ;{ }^{6} \mathrm{P}$ & 1.886 & \\
\hline & & 93138 & 45.29 & $3 d^{4} 5 p^{5} \mathrm{D} ;{ }^{6} \mathrm{~F}$ & 37.27 & $3 d^{3} 4 s 4 p^{4} F \cdot{ }^{3} \mathrm{P} ;{ }^{2} \mathrm{~F}$ & 1.069 & \\
\hline & 93143.853 & 93143 & 52.72 & $3 d^{4} 5 p^{5} \mathrm{D} ;{ }^{6} \mathrm{~F}$ & 32.10 & $3 d^{3} 4 s 4 p{ }^{4} F \cdot{ }^{3} \mathrm{P} ;{ }^{2} \mathrm{~F}$ & 1.103 & \\
\hline & 93670.984 & 93624 & 43.33 & $3 d^{4} 4 p^{3} F ;{ }^{4} D$ & 19.23 & $3 d^{4} 4 p{ }^{3} p ;{ }^{4} D$ & 1.380 & \\
\hline & 93776.125 & 93709 & 61.24 & $3 d^{4} 5 p^{5} \mathrm{D} ;{ }^{6} \mathrm{D}$ & 23.84 & $3 d^{3} 4 s$ p ${ }^{4}$ P. ${ }^{3} \mathrm{P} ;{ }^{6} \mathrm{D}$ & 1.650 & \\
\hline & 93973.992 & 93909 & 44.72 & $3 d^{4} 5 p^{5} D ;{ }^{6} P$ & 21.54 & $3 d^{3} 4 s 4 p{ }^{4} \mathrm{P} .{ }^{3} \mathrm{P} ;{ }^{6} \mathrm{P}$ & & \\
\hline & 94144.430 & 94027 & 58.32 & $\begin{array}{l}3 \mathrm{~d}^{3} 4 \mathrm{~s} 4 \mathrm{p} \\
{ }^{4} \mathrm{P} \cdot{ }^{3} \mathrm{P} ;{ }^{6} \mathrm{D}\end{array}$ & 26.19 & $3 d^{4} 5 p^{5} \mathrm{D} ;{ }^{6} \mathrm{D}$ & 1.679 & \\
\hline & 94265.930 & 94246 & 64.23 & $3 d^{4} 5 p^{5} D ;{ }^{4} P$ & 15.20 & $3 d^{4} 5 p^{5} D ;{ }^{6} P$ & 1.627 & \\
\hline & 94365.203 & 94407 & 87.99 & $3 d^{4} 5 p^{5} D ;{ }^{4} F$ & 4.40 & $3 d^{4} 5 p^{5} \mathrm{D} ;{ }^{4} \mathrm{P}$ & 1.081 & \\
\hline & 95076.719 & 94993 & 82.35 & $3 d^{4} 5 p^{5} D ;{ }^{4} D$ & 4.41 & $3 d^{3} 4 s 4{ }^{4} F \cdot{ }^{1} \mathrm{P} ;{ }^{4} \mathrm{D}$ & 1.368 & \\
\hline & 97070.938 & 97285 & 84.37 & $\begin{array}{l}3 \mathrm{~d}^{3} 4 \mathrm{~s} 4 \mathrm{p} \\
{ }^{2} \mathrm{G} \cdot{ }^{3} \mathrm{P} ;{ }^{4} \mathrm{G}\end{array}$ & 7.72 & $3 d^{3} 4 s 4 p{ }^{4} F .{ }^{1} P ;{ }^{4} G$ & 0.574 & \\
\hline & 97182.555 & 97310 & 47.66 & $\begin{array}{l}3 \mathrm{~d}^{3} 4 \mathrm{~s} 4 \mathrm{p} \\
{ }^{4} \mathrm{P} .{ }^{3} \mathrm{P} ;{ }^{4} \mathrm{P}\end{array}$ & 38.83 & $3 d^{3} 4 s 4 p{ }^{4} P .{ }^{3} P ;{ }^{6} S$ & 1.756 & \\
\hline & 97875.016 & 97864 & 60.60 & $\begin{array}{l}3 d^{3} 4 s 4 p \\
{ }^{4} \mathrm{P} .{ }^{3} \mathrm{P} ;{ }^{6} \mathrm{~S}\end{array}$ & 31.99 & $3 d^{3} 4 s 4 p{ }^{4} P .{ }^{3} P ;{ }^{4} \mathrm{P}$ & 1.841 & \\
\hline & 98207.461 & 98021 & 51.59 & $3 d^{4} 4 p^{3} F ;{ }^{2} D$ & 18.56 & $3 d^{4} 4 p^{3} F ;{ }^{2} D$ & 1.196 & \\
\hline & 98641.891 & 98756 & 57.67 & $\begin{array}{l}3 d^{3} 4 s 4 p \\
{ }^{2} \mathrm{G} \cdot{ }^{3} \mathrm{P} ;{ }^{4} \mathrm{~F}\end{array}$ & 14.13 & $3 d^{4} 4 p^{1} G ;{ }^{2} F$ & 0.985 & \\
\hline & 99243.938 & 98911 & 35.46 & $3 d^{4} 4 p^{1} G ;{ }^{2} F$ & 21.18 & $3 d^{3} 4 s 4 p^{2} G \cdot{ }^{3} \mathrm{P} ;{ }^{4} \mathrm{~F}$ & 0.907 & \\
\hline & & 100798 & 52.35 & $\begin{array}{l}3 \mathrm{~d}^{3} 4 \mathrm{~s} 4 \mathrm{p} \\
{ }^{2} \mathrm{P} .{ }^{3} \mathrm{P} ;{ }^{4} \mathrm{P}\end{array}$ & 27.08 & $3 d^{3} 4 s 4 p^{2}$ D. ${ }^{3} \mathrm{P} ;{ }^{4} \mathrm{P}$ & 1.586 & \\
\hline & 101074.523 & 100857 & 45.68 & $\begin{array}{l}3 \mathrm{~d}^{3} 4 \mathrm{~s} 4 \mathrm{p} \\
{ }^{2} \mathrm{P} .{ }^{3} \mathrm{P} ;{ }^{4} \mathrm{D}\end{array}$ & 29.69 & $3 d^{3} 4 s 4 p{ }^{4} P .{ }^{3} \mathrm{P} ;{ }^{4} \mathrm{D}$ & 1.367 & \\
\hline & 102145.641 & 102220 & 67.33 & $\begin{array}{l}3 d^{3} 4 s 4 p \\
{ }^{2} \mathrm{G} \cdot{ }^{3} \mathrm{P} ;{ }^{2} \mathrm{~F}\end{array}$ & 6.99 & $3 d^{4} 4 p{ }^{1} G ;{ }^{2} F$ & 0.877 & \\
\hline & 102297.062 & 102442 & 41.13 & $\begin{array}{l}3 \mathrm{~d}^{3} 4 \mathrm{~s} 4 \mathrm{p} \\
{ }^{2} \mathrm{D} \cdot{ }^{3} \mathrm{P} ;{ }^{4} \mathrm{~F}\end{array}$ & 12.77 & $3 d^{3} 4 s 4{ }^{2} G \cdot{ }^{3} \mathrm{P} ;{ }^{4} \mathrm{~F}$ & 1.042 & \\
\hline & 102655.906 & 102815 & 43.71 & $\begin{array}{l}3 d^{3} 4 s 4 p \\
{ }^{4} \mathrm{P} .{ }^{3} \mathrm{P} ;{ }^{4} \mathrm{D}\end{array}$ & 40.54 & $3 \mathrm{~d}^{3} 4 \mathrm{~s} 4 \mathrm{p}^{2} \mathrm{P} .{ }^{3} \mathrm{P} ;{ }^{4} \mathrm{D}$ & 1.350 & \\
\hline & 102678.945 & 102877 & 76.41 & $\begin{array}{l}3 d^{3} 4 s 4 p \\
{ }^{4} \mathrm{~F} .{ }^{1} \mathrm{P} ;{ }^{4} \mathrm{G}\end{array}$ & 8.15 & $3 \mathrm{~d}^{3} 4 \mathrm{~s} 4 \mathrm{p}^{2} \mathrm{G} \cdot{ }^{3} \mathrm{P} ;{ }^{4} \mathrm{G}$ & 0.574 & \\
\hline & & 103278 & 45.91 & $\begin{array}{l}3 d^{3} 4 s 4 p \\
{ }^{2} \mathrm{P} \cdot{ }^{3} \mathrm{P} ;{ }^{2} \mathrm{D}\end{array}$ & 24.43 & $3 \mathrm{~d}^{3} 4 \mathrm{~s} 4 \mathrm{p}^{2} \mathrm{D} \cdot{ }^{3} \mathrm{P} ;{ }^{2} \mathrm{D}$ & 1.206 & \\
\hline & 104467.805 & 104149 & 44.48 & $\begin{array}{l}3 d^{3} 4 s 4 p \\
{ }^{2} D \cdot{ }^{3} P ;{ }^{4} D\end{array}$ & 28.24 & $3 d^{3} 4 s 4 p{ }^{4} F \cdot{ }^{1} P ;{ }^{4} D$ & 1.372 & \\
\hline
\end{tabular}


Table 5 (continued)

\begin{tabular}{|c|c|c|c|c|c|c|c|c|}
\hline \multirow[t]{2}{*}{$J$-value } & \multirow{2}{*}{$\begin{array}{c}E_{o b s} \\
\left(\mathrm{~cm}^{-1}\right) \\
{[8,27]} \\
104869.117\end{array}$} & \multirow{2}{*}{$\begin{array}{c}\begin{array}{c}E_{\text {calc }} \\
\left(\mathrm{cm}^{-1}\right)\end{array} \\
104755\end{array}$} & \multicolumn{2}{|c|}{$\begin{array}{l}\text { Largest eigenvalue component } \\
(\%)\end{array}$} & \multicolumn{2}{|c|}{$\begin{array}{c}\text { Next largest eigenvalue component } \\
(\%)\end{array}$} & \multirow{2}{*}{$\begin{array}{r}\text { Calc. } g_{J} \\
1.364\end{array}$} & \multirow{2}{*}{$\begin{array}{c}\text { Obs. } g_{J} \\
{[5]} \\
\end{array}$} \\
\hline & & & 36.92 & $\begin{array}{l}3 d^{3} 4 s 4 p \\
{ }^{4} \mathrm{~F} .{ }^{1} \mathrm{P} ;{ }^{4} \mathrm{D}\end{array}$ & 29.14 & $3 d^{3} 4 s 4 p^{2} D \cdot{ }^{3} P ;{ }^{4} D$ & & \\
\hline & \multirow[t]{6}{*}{105121.969} & 105111 & 35.47 & $3 d^{4} 4 f^{5} D ;{ }^{4} P$ & 22.71 & $3 d^{4} 4 f^{5} D ;{ }^{4} F$ & 1.263 & \\
\hline & & 105139 & 38.74 & $3 d^{4} 4 f^{5} D ;{ }^{6} H$ & 34.64 & $3 d^{4} 4 f^{5} D ;{ }^{4} P$ & 0.976 & \\
\hline & & 105146 & 54.25 & $3 d^{4} 4 f^{5} D ;{ }^{6} H$ & 12.76 & $3 d^{4} 4 f^{5} D ;{ }^{4} F$ & 0.710 & \\
\hline & & 105289 & 39.12 & $3 d^{4} 4 f^{5} D ;{ }^{6} D$ & 33.09 & $3 d^{4} 4 f^{5} D ;{ }^{6} P$ & 1.641 & \\
\hline & & 105425 & 35.13 & $3 d^{4} 4 f^{5} D ;{ }^{6} G$ & 31.57 & $3 d^{4} 4 f^{5} D ;{ }^{6} F$ & 1.286 & \\
\hline & & 105470 & 38.03 & $\begin{array}{l}3 \mathrm{~d}^{3} 4 \mathrm{~s} 4 \mathrm{p} \\
2 \mathrm{P} \cdot{ }^{3} \mathrm{P} ;{ }^{4} \mathrm{P}\end{array}$ & 35.43 & $3 d^{3} 4 s 4 p^{2}$ D. ${ }^{3} P ;{ }^{4} P$ & 1.587 & \\
\hline & 105438.273 & 105561 & 46.61 & $3 d^{4} 4 f^{5} D ;{ }^{6} G$ & 17.89 & $3 d^{4} 4 f^{5} D ;{ }^{6} D$ & 1.256 & \\
\hline & \multirow[t]{2}{*}{105532.070} & 105631 & 60.64 & $3 d^{4} 4 f^{5} D ;{ }^{4} D$ & 11.14 & $3 d^{4} 4 f^{5} D ;{ }^{6} P$ & 1.410 & \\
\hline & & 105720 & 40.17 & $3 d^{4} 4 f^{5} D ;{ }^{6} F$ & 30.90 & $3 d^{4} 4 f^{5} D ;{ }^{6} D$ & 1.358 & \\
\hline & 105677.609 & 105735 & 72.04 & $3 d^{4} 4 f^{5} D ;{ }^{4} G$ & 7.34 & $\begin{array}{l}3 \mathrm{~d}^{3} 4 \mathrm{~s} 4 \mathrm{p}^{2} \mathrm{H} \\
{ }^{3} \mathrm{P} ;{ }^{4} \mathrm{G}\end{array}$ & 0.693 & \\
\hline & \multirow[t]{2}{*}{105902.969} & 106054 & 42.79 & $3 d^{4} 4 f^{5} D ;{ }^{4} F$ & 21.65 & $3 d^{3} 4 s 4 p^{4} \mathrm{~F} .{ }^{1} \mathrm{P} ;{ }^{4} \mathrm{~F}$ & 0.951 & \\
\hline & & 106170 & 60.26 & $\begin{array}{l}3 \mathrm{~d}^{3} 4 \mathrm{~s} 4 \mathrm{p} \\
{ }^{2} \mathrm{H} \cdot{ }^{3} \mathrm{P} ;{ }^{4} \mathrm{G}\end{array}$ & 9.79 & $3 d^{4} 4 f^{5} D ;{ }^{4} G$ & 0.656 & \\
\hline & 107022.148 & 106930 & 46.88 & $\begin{array}{l}3 d^{3} 4 s 4 p \\
{ }^{2} \mathrm{D} \cdot{ }^{3} \mathrm{P} ;{ }^{2} \mathrm{~F}\end{array}$ & 27.17 & $3 d^{3} 4 s 4 p{ }^{4} P .{ }^{3} \mathrm{P} ;{ }^{2} \mathrm{D}$ & 0.959 & \\
\hline & 107355.516 & 107244 & 63.20 & $\begin{array}{l}3 d^{3} 4 s 4 p \\
{ }^{4} \mathrm{P} \cdot{ }^{3} \mathrm{P} ;{ }^{2} \mathrm{D}\end{array}$ & 18.80 & $3 d^{3} 4 s 4 p^{2}$ D. ${ }^{3} \mathrm{P} ;{ }^{2} \mathrm{~F}$ & 1.098 & \\
\hline & 109694.297 & 109633 & 96.24 & $3 \mathrm{~d}^{4} 6 \mathrm{p}^{5} \mathrm{D} ;{ }^{6} \mathrm{~F}$ & 1.51 & $3 \mathrm{~d}^{4} 6 \mathrm{p}^{5} \mathrm{D} ;{ }^{4} \mathrm{~F}$ & 1.314 & \\
\hline & \multirow{2}{*}{109864.891} & 109769 & 42.29 & $3 d^{4} 6 p^{5} D ;{ }^{6} P$ & 34.27 & $3 \mathrm{~d}^{4} 6 \mathrm{p}^{5} \mathrm{D} ;{ }^{4} \mathrm{P}$ & 1.730 & \\
\hline & & 109997 & 61.99 & $3 d^{4} 6 p^{5} D ;{ }^{6} D$ & 34.84 & $3 \mathrm{~d}^{4} 6 \mathrm{p}^{5} \mathrm{D} ;{ }^{6} \mathrm{P}$ & 1.726 & \\
\hline & 109943.383 & 110057 & 43.71 & $\begin{array}{l}3 d^{3} 4 s 4 p \\
{ }^{2} \mathrm{D} \cdot{ }^{3} \mathrm{P} ;{ }^{2} \mathrm{D}\end{array}$ & 39.50 & $3 d^{3} 4 s 4 p^{2} P \cdot{ }^{3} P ;{ }^{2} D$ & 1.202 & \\
\hline & \multirow[t]{2}{*}{109973.973} & 110194 & 53.11 & $3 \mathrm{~d}^{4} 6 \mathrm{p}^{5} \mathrm{D} ;{ }^{4} \mathrm{P}$ & 21.81 & $3 d^{4} 6 p^{5} \mathrm{D} ;{ }^{6} \mathrm{P}$ & 1.656 & \\
\hline & & 110350 & 76.94 & $3 d^{4} 5 p^{3} H ;{ }^{4} G$ & 11.07 & $3 d^{4} 6 p^{5} D ;{ }^{4} F$ & 0.640 & \\
\hline & \multirow[t]{2}{*}{110315.062} & 110396 & 75.77 & $3 \mathrm{~d}^{4} 6 \mathrm{p}^{5} \mathrm{D} ;{ }^{4} \mathrm{~F}$ & 12.02 & $3 d^{4} 5 p^{3} \mathrm{H} ;{ }^{4} \mathrm{G}$ & 1.000 & \\
\hline & & 110823 & 47.79 & $3 d^{4} 5 p^{3} P ;{ }^{4} \mathrm{D}$ & 32.61 & $3 d^{4} 5 p^{3} P ;{ }^{4} D$ & 1.379 & \\
\hline & \multirow[t]{14}{*}{111082.312} & 111171 & 83.53 & $3 d^{4} 6 p^{5} D ;{ }^{4} D$ & 5.12 & $3 d^{3} 4 s 4 p^{4} F \cdot{ }^{1} \mathrm{P} ;{ }^{4} \mathrm{D}$ & 1.371 & \\
\hline & & 111394 & 70.79 & $3 d^{4} 5 p^{3} F ;{ }^{4} G$ & 21.54 & $3 d^{4} 5 p^{3} F ;{ }^{4} G$ & 0.590 & \\
\hline & & 111430 & 45.94 & $3 d^{4} 5 p^{3} \mathrm{P} ;{ }^{4} \mathrm{P}$ & 30.14 & $3 d^{4} 5 p^{3} P ;{ }^{4} \mathrm{P}$ & 1.536 & \\
\hline & & 111835 & 33.34 & $3 d^{4} 5 p^{3} F ;{ }^{4} F$ & 18.85 & $3 d^{4} 5 p^{3} F ;{ }^{2} D$ & 1.122 & \\
\hline & & 112122 & 37.80 & $3 d^{4} 5 p^{3} p ;{ }^{2} D$ & 25.68 & $3 d^{4} 5 p^{3} p ;{ }^{2} D$ & 1.203 & \\
\hline & & 112140 & 28.44 & $3 d^{4} 5 p^{3} F ;{ }^{4} F$ & 13.29 & $3 d^{4} 5 p^{3} F ;{ }^{2} F$ & 1.085 & \\
\hline & & 112264 & 37.84 & $3 d^{4} 5 p^{3} F ;{ }^{2} F$ & 27.21 & $3 d^{4} 5 p^{3} F ;{ }^{4} D$ & 1.071 & \\
\hline & & 112298 & 46.41 & $3 d^{4} 5 p^{3} F ;{ }^{2} D$ & 14.09 & $3 d^{4} 5 p^{3} F ;{ }^{2} D$ & 1.197 & \\
\hline & & 113107 & 60.39 & $3 \mathrm{~d}^{4} 4 \mathrm{p}{ }^{1} \mathrm{D} ;{ }^{2} \mathrm{~F}$ & 17.19 & $3 d^{4} 4 p{ }^{1} D ;{ }^{2} F$ & 0.861 & \\
\hline & & 113985 & 43.43 & $3 d^{4} 5 p^{3} G ;{ }^{4} G$ & 24.93 & $3 d^{4} 5 p^{3} G ;{ }^{4} F$ & 0.796 & \\
\hline & & 114198 & 39.32 & $3 d^{4} 5 p^{3} G ;{ }^{4} G$ & 27.41 & $3 d^{3} 4 s 4 p^{2} F \cdot{ }^{3} \mathrm{P} ;{ }^{4} \mathrm{~F}$ & 0.839 & \\
\hline & & 114515 & 68.91 & $3 d^{4} 5 p^{3} G ;{ }^{2} F$ & 11.20 & $3 d^{4} 5 p^{3} G ;{ }^{4} G$ & 0.837 & \\
\hline & & 115082 & 52.38 & $3 d^{4} 5 f^{5} D ;{ }^{4} P$ & 10.98 & $3 d^{4} 5 f^{5} D ;{ }^{4} D$ & 1.447 & \\
\hline & & 115117 & 38.60 & $\begin{array}{l}3 \mathrm{~d}^{3} 4 \mathrm{~s} 4 \mathrm{p} \\
{ }^{2} \mathrm{~F} \cdot{ }^{3} \mathrm{P} ;{ }^{4} \mathrm{~F}\end{array}$ & 28.81 & $3 d^{4} 5 p^{3} G ;{ }^{4} F$ & 1.149 & \\
\hline & 115208.312 & 115234 & 90.58 & $3 d^{4} 5 f^{5} D ;{ }^{6} H$ & 6.47 & $3 d^{4} 5 f^{5} D ;{ }^{6} G$ & 0.344 & \\
\hline & \multirow[t]{2}{*}{115309.562} & 115340 & 38.23 & $3 d^{4} 5 f^{5} D ;{ }^{6} D$ & 30.48 & $3 d^{4} 5 f^{5} D ;{ }^{6} F$ & 1.448 & \\
\hline & & 115426 & 24.94 & $3 d^{4} 5 f^{5} D ;{ }^{4} D$ & 23.38 & $3 d^{4} 4 p^{5} D ;{ }^{6} G$ & 1.231 & \\
\hline & \multirow[t]{2}{*}{115461.742} & 115486 & 26.03 & $3 d^{4} 5 f^{5} D ;{ }^{4} G$ & 23.51 & $3 d^{4} 4 p^{5} D ;{ }^{6} G$ & 1.068 & \\
\hline & & 115580 & 28.63 & $3 d^{4} 5 f^{5} D ;{ }^{4} G$ & 24.43 & $3 d^{4} 4 p^{5} D ;{ }^{6} G$ & 1.017 & \\
\hline & 115767.047 & 115618 & 27.97 & $3 d^{4} 5 f^{5} D ;{ }^{4} G$ & 26.78 & $3 d^{4} 4 p^{5} D ;{ }^{6} P$ & 1.237 & \\
\hline & & 115722 & 69.55 & $3 d^{4} 4 p^{1} \mathrm{D} ;{ }^{2} \mathrm{D}$ & 23.99 & $3 d^{4} 4 p^{1} D ;{ }^{2} D$ & 1.199 & \\
\hline & & 115765 & 44.58 & $3 d^{4} 5 f^{5} D ;{ }^{6} D$ & 28.61 & $3 d^{4} 5 f^{5} D ;{ }^{6} P$ & 1.614 & \\
\hline & 115810.297 & 115817 & 55.26 & $3 d^{4} 5 f^{5} D ;{ }^{4} F$ & 16.85 & $3 d^{4} 5 f^{5} D ;{ }^{4} D$ & 1.045 & \\
\hline & & 116243 & 64.53 & $\begin{array}{l}3 d^{3} 4 s 4 p \\
{ }^{4} \mathrm{P} .{ }^{1} \mathrm{P} ;{ }^{4} \mathrm{P}\end{array}$ & 21.19 & $3 d^{4} 5 f^{5} D ;{ }^{4} P$ & 1.597 & \\
\hline & & 116672 & 93.95 & $\begin{array}{l}3 d^{3} 4 s 4 p \\
{ }^{2} \mathrm{~F} \cdot{ }^{3} \mathrm{P} ;{ }^{4} \mathrm{G}\end{array}$ & 3.76 & $\begin{array}{c}3 d^{3} 4 s 4 p^{2} \mathrm{H} \\
{ }^{3} \mathrm{P} ;{ }^{4} \mathrm{G}\end{array}$ & 0.574 & \\
\hline & & 117131 & 55.24 & $\begin{array}{l}3 d^{3} 4 s 4 p \\
{ }^{2} \mathrm{~F}^{3} \mathrm{P} ;{ }^{4} \mathrm{D}\end{array}$ & 29.93 & $3 d^{3} 4 s$ p ${ }^{4} P .{ }^{1} \mathrm{P} ;{ }^{4} \mathrm{D}$ & 1.372 & \\
\hline & & 118054 & 36.66 & $3 d^{4} 5 p{ }^{1} G ;{ }^{2} F$ & 26.39 & $3 d^{3} 4 s 4 p^{2}$ G. ${ }^{1} \mathrm{P} ;{ }^{2} \mathrm{~F}$ & 0.858 & \\
\hline & & 118788 & 50.83 & $\begin{array}{l}3 d^{3} 4 s 4 p \\
{ }^{4} \mathrm{P} .{ }^{1} \mathrm{P} ;{ }^{4} \mathrm{D}\end{array}$ & 36.95 & $3 d^{3} 4 s 4 p{ }^{2} F \cdot{ }^{3} P ;{ }^{4} D$ & 1.370 & \\
\hline & & 119248 & 30.82 & $\begin{array}{l}3 \mathrm{~d}^{3} 4 \mathrm{~s} 4 \mathrm{p} \\
{ }^{2} \mathrm{G} \cdot{ }^{1} \mathrm{P} ;{ }^{2} \mathrm{~F}\end{array}$ & 26.33 & $3 d^{4} 5 p^{3} \mathrm{D} ;{ }^{2} \mathrm{~F}$ & 0.859 & \\
\hline & & 120046 & 51.79 & $\begin{array}{l}3 d^{3} 4 s 4 p \\
{ }^{2} \mathrm{~F}^{3} \mathrm{P} ;{ }^{2} \mathrm{D}\end{array}$ & 16.03 & $3 d^{4} 5 p^{3} D ;{ }^{2} D$ & 1.202 & \\
\hline & & 120365 & 76.53 & $3 d^{4} 5 p^{3} \mathrm{D} ;{ }^{4} \mathrm{~F}$ & 16.42 & $3 d^{4} 5 p^{3} D ;{ }^{4} D$ & 1.093 & \\
\hline & & 120433 & 34.72 & $3 d^{4} 5 p^{3} D ;{ }^{2} D$ & 23.53 & $3 d^{3} 4 s 4 p^{2} F \cdot{ }^{3} \mathrm{P} ;{ }^{2} \mathrm{D}$ & 1.261 & \\
\hline & & 120726 & 48.34 & $3 d^{4} 5 p^{3} D ;{ }^{4} D$ & 17.51 & $3 d^{4} 5 p^{3} \mathrm{D} ;{ }^{4} \mathrm{~F}$ & 1.273 & \\
\hline & & 120849 & 76.78 & $3 d^{4} 4 f^{3} H ;{ }^{4} G$ & 17.99 & $3 d^{4} 4 f^{3} H ;{ }^{2} F$ & 0.643 & \\
\hline
\end{tabular}


Table 5 (continued)

\begin{tabular}{|c|c|c|c|c|c|c|c|c|}
\hline \multirow[t]{3}{*}{$J$-value } & \multirow{3}{*}{$\begin{array}{c}E_{o b s} \\
\left(\mathrm{~cm}^{-1}\right) \\
{[8,27]}\end{array}$} & \multirow{2}{*}{$\begin{array}{c}\begin{array}{c}E_{\text {calc }} \\
\left(\mathrm{cm}^{-1}\right)\end{array} \\
120896\end{array}$} & \multicolumn{2}{|c|}{$\begin{array}{l}\text { Largest eigenvalue component } \\
\text { (\%) }\end{array}$} & \multicolumn{2}{|c|}{$\begin{array}{l}\text { Next largest eigenvalue component } \\
(\%)\end{array}$} & Calc. $g_{J}$ & $\begin{array}{c}\text { Obs. } g_{J} \\
{[5]}\end{array}$ \\
\hline & & & 54.24 & $3 \mathrm{~d}^{4} 4 \mathrm{f}^{3} \mathrm{H} ;{ }^{2} \mathrm{~F}$ & 23.60 & $3 d^{4} 4 f^{3} H ;{ }^{4} F$ & 0.899 & \\
\hline & & 120992 & 70.02 & $3 d^{4} 5 p^{3} D ;{ }^{4} P$ & 6.87 & $3 d^{4} 4 f^{3} H ;{ }^{4} D$ & 1.509 & \\
\hline \multicolumn{9}{|l|}{3.5} \\
\hline & 47227.219 & 47165 & 99.53 & $3 d^{4} 4 p^{5} \mathrm{D} ;{ }^{6} \mathrm{~F}$ & 0.24 & $3 \mathrm{~d}^{4} 5 \mathrm{p}^{5} \mathrm{D} ;{ }^{6} \mathrm{~F}$ & 1.398 & \\
\hline & 48632.059 & 48559 & 99.56 & $3 d^{4} 4 p^{5} \mathrm{D} ;{ }^{6} \mathrm{P}$ & 0.13 & $3 d^{4} 5 p^{5} \mathrm{D} ;{ }^{6} \mathrm{P}$ & 1.715 & \\
\hline & 49645.805 & 49639 & 99.30 & $3 d^{4} 4 p^{5} D ;{ }^{6} D$ & 0.31 & $3 d^{4} 4 p^{5} D ;{ }^{4} F$ & 1.587 & \\
\hline & 51788.816 & 51980 & 95.34 & $3 d^{4} 4 p^{5} D ;{ }^{4} F$ & 1.80 & $3 d^{4} 4 p^{3} F ;{ }^{4} F$ & 1.240 & \\
\hline & 54784.449 & 54707 & 97.20 & $3 d^{4} 4 p^{5} \mathrm{D} ;{ }^{4} \mathrm{D}$ & 0.92 & $3 d^{4} 4 p^{3} D ;{ }^{4} D$ & 1.429 & \\
\hline & 63600.863 & 63642 & 82.23 & $3 d^{4} 4 p^{3} \mathrm{H} ;{ }^{4} \mathrm{H}$ & 16.09 & $3 d^{4} 4 p^{3} G ;{ }^{4} \mathrm{H}$ & 0.669 & \\
\hline & 64924.461 & 64801 & 44.80 & $3 d^{4} 4 p^{3} p ;{ }^{4} D$ & 31.52 & $3 d^{4} 4 p^{3} p ;{ }^{4} D$ & 1.428 & \\
\hline & 65256.852 & 65298 & 31.00 & $3 d^{4} 4 p^{3} \mathrm{H} ;{ }^{2} \mathrm{G}$ & 22.87 & $3 d^{4} 4 p{ }^{3} F ;{ }^{4} G$ & 0.934 & \\
\hline & 65542.898 & 65498 & 27.66 & $3 d^{4} 4 p^{3} \mathrm{H} ;{ }^{2} \mathrm{G}$ & 27.29 & $3 d^{4} 4 p^{3} F ;{ }^{4} G$ & 0.939 & \\
\hline & 67333.781 & 67323 & 75.03 & $3 d^{4} 4 p^{3} F ;{ }^{4} F$ & 14.53 & $3 d^{4} 4 p^{3} F ;{ }^{4} F$ & 1.228 & \\
\hline & 67393.453 & 67378 & 69.21 & $3 d^{4} 4 p^{3} \mathrm{H} ;{ }^{4} \mathrm{G}$ & 17.47 & $3 d^{4} 4 p^{3} F ;{ }^{4} G$ & 0.996 & \\
\hline & 67875.367 & 68035 & 50.77 & $3 d^{4} 4 p^{3} F ;{ }^{4} D$ & 14.36 & $3 d^{4} 4 p^{3} F ;{ }^{4} D$ & 1.393 & \\
\hline & 68759.805 & 68714 & 33.51 & $3 d^{4} 4 p{ }^{3} F ;{ }^{2} F$ & 25.44 & $3 d^{4} 4 p^{3} G ;{ }^{2} F$ & 1.165 & \\
\hline & 68843.273 & 68938 & 82.37 & $3 d^{4} 4 p^{3} G ;{ }^{4} H$ & 16.12 & $3 d^{4} 4 p^{3} \mathrm{H} ;{ }^{4} \mathrm{H}$ & 0.669 & \\
\hline & 69506.070 & 69621 & 76.20 & $3 d^{4} 4 p{ }^{3} G ;{ }^{4} F$ & 9.02 & $3 d^{4} 4 p^{3} D ;{ }^{4} F$ & 1.210 & \\
\hline & 69903.477 & 69778 & 36.09 & $3 d^{4} 4 p{ }^{3} F ;{ }^{2} G$ & 24.67 & $3 d^{4} 4 p^{3} H ;{ }^{2} G$ & 0.911 & \\
\hline & 70426.992 & 70316 & 69.91 & $3 d^{4} 4 p{ }^{3} G ;{ }^{4} G$ & 12.89 & $3 d^{4} 4 p^{3} H ;{ }^{4} G$ & 0.999 & \\
\hline & 70852.164 & 70695 & 52.42 & $3 d^{4} 4 p^{3} G ;{ }^{2} F$ & 33.12 & $3 d^{4} 4 p^{3} F ;{ }^{2} F$ & 1.142 & \\
\hline & 72648.516 & 72752 & 78.63 & $3 d^{4} 4 p^{3} G ;{ }^{2} G$ & 10.37 & $3 d^{4} 4 p^{3} \mathrm{H} ;{ }^{2} \mathrm{G}$ & 0.893 & \\
\hline & 73485.609 & 73623 & 92.77 & $3 d^{4} 4 p^{3} D ;{ }^{4} D$ & 2.62 & $3 d^{4} 4 p^{3} D ;{ }^{4} F$ & 1.421 & \\
\hline & 74114.367 & 74058 & 51.78 & $3 d^{4} 4 p{ }^{1} G ;{ }^{2} F$ & 24.62 & $3 d^{4} 4 p^{1} G ;{ }^{2} F$ & 1.145 & \\
\hline & 74423.672 & 74432 & 83.74 & $3 d^{4} 4 p{ }^{3} D ;{ }^{4} F$ & 10.17 & $3 d^{4} 4 p^{3} G ;{ }^{4} F$ & 1.242 & \\
\hline & 75716.495 & 75929 & 47.58 & $3 d^{4} 4 p{ }^{1} G ;{ }^{2} G$ & 28.11 & $3 d^{4} 4 p^{1} G ;{ }^{2} G$ & 0.891 & \\
\hline & 76878.953 & 76839 & 74.11 & $3 d^{4} 4 p{ }^{3} D ;{ }^{2} F$ & 7.47 & $3 d^{4} 4 p^{3} G ;{ }^{2} F$ & 1.143 & \\
\hline & & 79328 & 99.85 & $\begin{array}{l}3 d^{3} 4 s 4 p \\
{ }^{4} \mathrm{~F}^{3} \mathrm{P} ;{ }^{6} \mathrm{G}\end{array}$ & 0.03 & $3 d^{3} 4 s 4 p{ }^{4} F \cdot{ }^{3} P ;{ }^{4} F$ & 1.143 & \\
\hline & 81432.227 & 81376 & 69.36 & $3 d^{4} 4 p{ }^{1} D ;{ }^{2} F$ & 18.39 & $3 d^{4} 4 p{ }^{1} \mathrm{D} ;{ }^{2} \mathrm{~F}$ & 1.143 & \\
\hline & 81978.047 & 82032 & 76.16 & $\begin{array}{l}3 d^{3} 4 s 4 p \\
{ }^{4} \mathrm{~F}^{3} \mathrm{P} ;{ }^{6} \mathrm{D}\end{array}$ & 21.26 & $3 d^{3} 4 s 4 p{ }^{4} F \cdot{ }^{3} \mathrm{P} ;{ }^{6} \mathrm{~F}$ & 1.547 & \\
\hline & 82142.711 & 82126 & 78.30 & $\begin{array}{l}3 \mathrm{~d}^{3} 4 \mathrm{~s} 4 \mathrm{p} \\
{ }^{4} \mathrm{~F} .{ }^{3} \mathrm{P} ;{ }^{6} \mathrm{~F}\end{array}$ & 20.54 & $3 d^{3} 4 s 4 p^{4} F \cdot{ }^{3} P ;{ }^{6} D$ & 1.438 & \\
\hline & 84677.117 & 84456 & 87.25 & $3 d^{4} 4 p^{1} \mathrm{~F} ;{ }^{2} \mathrm{~F}$ & 3.21 & $3 d^{4} 4 p^{3} D ;{ }^{2} F$ & 1.141 & \\
\hline & 85573.125 & 85562 & 94.02 & $3 d^{4} 4 p{ }^{1} F ;{ }^{2} G$ & 1.26 & $3 d^{4} 4 p{ }^{1} G ;{ }^{2} G$ & 0.892 & \\
\hline & 86078.820 & 85981 & 89.48 & $\begin{array}{l}3 d^{3} 4 s 4 p \\
{ }^{4} \mathrm{~F} \cdot{ }^{3} \mathrm{P} ;{ }^{4} \mathrm{D}\end{array}$ & 4.67 & $3 d^{3} 4 s 4 p{ }^{4} P .{ }^{3} P ;{ }^{4} D$ & 1.430 & \\
\hline & 86797.242 & 86901 & 93.55 & $\begin{array}{l}3 d^{3} 4 s 4 p \\
{ }^{4} \mathrm{~F}^{3} \mathrm{P} ;{ }^{4} \mathrm{G}\end{array}$ & 3.79 & $3 d^{3} 4 s 4{ }^{2} G \cdot{ }^{3} P ;{ }^{4} G$ & 0.985 & \\
\hline & 87916.672 & 88239 & 74.86 & $\begin{array}{l}3 d^{3} 4 s 4 p \\
{ }^{4} \mathrm{~F} \cdot{ }^{3} \mathrm{P} ;{ }^{4} \mathrm{~F}\end{array}$ & 18.84 & $3 d^{4} 4 p^{3} F ;{ }^{4} F$ & 1.238 & \\
\hline & 90218.406 & 90078 & 56.38 & $3 d^{4} 4 p^{3} F ;{ }^{4} F$ & 19.63 & $3 d^{3} 4 s 4 p^{4} F \cdot{ }^{3} \mathrm{P} ;{ }^{4} \mathrm{~F}$ & 1.255 & \\
\hline & 90489.844 & 90388 & 32.67 & $3 d^{4} 4 p^{3} p ;{ }^{4} D$ & 23.42 & $3 d^{4} 4 p^{3} F ;{ }^{4} D$ & 1.406 & \\
\hline & 90830.844 & 91122 & 43.29 & $3 d^{4} 4 p{ }^{3} F ;{ }^{2} F$ & 16.63 & $3 d^{4} 4 p{ }^{3} F ;{ }^{4} G$ & 1.108 & \\
\hline & 91122.859 & 91306 & 55.09 & $3 d^{4} 4 p{ }^{3} F ;{ }^{4} G$ & 18.26 & $3 d^{4} 4 p{ }^{3} F ;{ }^{4} G$ & 1.024 & \\
\hline & & 91658 & 89.81 & $\begin{array}{l}3 d^{3} 4 s 4 p \\
{ }^{4} \mathrm{~F}^{3} \mathrm{P} ;{ }^{2} \mathrm{G}\end{array}$ & 4.61 & $3 d^{4} 4 p^{3} F ;{ }^{2} G$ & 0.892 & \\
\hline & 92653.172 & 92738 & 68.21 & $\begin{array}{l}3 \mathrm{~d}^{3} 4 \mathrm{~s} 4 \mathrm{p} \\
{ }^{4} \mathrm{P} \cdot{ }^{3} \mathrm{P} ;{ }^{6} \mathrm{P}\end{array}$ & 31.12 & $3 d^{4} 5 p^{5} D ;{ }^{6} P$ & 1.715 & \\
\hline & 93276.853 & 93312 & 97.94 & $3 d^{4} 5 p^{5} \mathrm{D} ;{ }^{6} \mathrm{~F}$ & 0.85 & $3 d^{4} 5 p^{5} D ;{ }^{4} F$ & 1.397 & \\
\hline & & 93458 & 66.06 & $\begin{array}{l}3 d^{3} 4 s 4 p \\
{ }^{4} F^{3} P ;{ }^{2} F\end{array}$ & 8.33 & $3 d^{4} 4 p^{3} F ;{ }^{2} F$ & 1.191 & \\
\hline & 93531.695 & 93602 & 38.12 & $3 d^{4} 4 p^{3} F ;{ }^{4} D$ & 16.18 & $3 d^{4} 4 p^{3} P ;{ }^{4} D$ & 1.380 & \\
\hline & 93801.523 & 93833 & 72.90 & $3 d^{4} 5 p^{5} D ;{ }^{6} D$ & 22.47 & $3 d^{3} 4 s 4 p{ }^{4} P \cdot{ }^{3} P ;{ }^{6} D$ & 1.582 & \\
\hline & 93966.430 & 94116 & 65.37 & $3 d^{4} 5 p^{5} \mathrm{D} ;{ }^{6} \mathrm{P}$ & 30.80 & $3 d^{3} 4 s 4 p{ }^{4} \mathrm{P} .{ }^{3} \mathrm{P} ;{ }^{6} \mathrm{P}$ & 1.705 & \\
\hline & 94218.672 & 94236 & 73.12 & $\begin{array}{l}3 d^{3} 4 s 4 p \\
{ }^{4} \mathrm{P} .{ }^{3} \mathrm{P} ;{ }^{6} \mathrm{D}\end{array}$ & 21.19 & $3 d^{4} 5 p^{5} D ;{ }^{6} D$ & 1.574 & \\
\hline & 94452.672 & 94356 & 71.43 & $3 d^{4} 4 p{ }^{3} F ;{ }^{2} G$ & 20.30 & $3 d^{4} 4 p^{3} F ;{ }^{2} G$ & 0.889 & \\
\hline & 94522.305 & 94518 & 87.33 & $3 d^{4} 5 p^{5} D ;{ }^{4} F$ & 4.85 & $3 d^{4} 5 p^{5} D ;{ }^{6} D$ & 1.271 & \\
\hline & 95250.680 & 95137 & 84.67 & $3 d^{4} 5 p^{5} D ;{ }^{4} D$ & 4.36 & $3 d^{3} 4 s$ 4p ${ }^{4}$ F. ${ }^{1} \mathrm{P} ;{ }^{4} \mathrm{D}$ & 1.429 & \\
\hline & & 96229 & 83.29 & $\begin{array}{l}3 d^{3} 4 s 4 p \\
{ }^{2} \mathrm{G} \cdot{ }^{3} \mathrm{P} ;{ }^{4} \mathrm{H}\end{array}$ & 15.04 & $\begin{array}{c}3 \mathrm{~d}^{3} 4 \mathrm{~s} 4 \mathrm{p}^{2} \mathrm{H} \\
{ }^{3} \mathrm{P} ;{ }^{4} \mathrm{H}\end{array}$ & 0.670 & \\
\hline & 97187.102 & 97262 & 49.60 & $3 d^{4} 4 p{ }^{1} G ;{ }^{2} G$ & 27.94 & $3 d^{4} 4 p^{1} G ;{ }^{2} G$ & 0.898 & \\
\hline & 97728.227 & 97450 & 77.85 & $\begin{array}{l}3 d^{3} 4 s 4 p \\
{ }^{2} \mathrm{G} \cdot{ }^{3} \mathrm{P} ;{ }^{4} \mathrm{G}\end{array}$ & 6.77 & $3 d^{3} 4 s 4 p{ }^{4} F \cdot{ }^{1} P ;{ }^{4} G$ & 0.975 & \\
\hline & 98719.328 & 98578 & 46.29 & $3 d^{4} 4 p{ }^{1} G ;{ }^{2} F$ & 20.34 & $3 d^{4} 4 p^{1} G ;{ }^{2} F$ & 1.145 & \\
\hline & 99069.273 & 98959 & 76.87 & $\begin{array}{l}3 d^{3} 4 s 4 p \\
{ }^{2} \mathrm{G} \cdot{ }^{3} \mathrm{P} ;{ }^{4} \mathrm{~F}\end{array}$ & 8.89 & $3 d^{3} 4 s$ p ${ }^{2}$ D. ${ }^{3} \mathrm{P} ;{ }^{4} \mathrm{~F}$ & 1.231 & \\
\hline & 101514.195 & 101244 & 44.52 & $\begin{array}{l}3 d^{3} 4 s 4 p \\
{ }^{2} \mathrm{P} \cdot{ }^{3} \mathrm{P} ;{ }^{4} \mathrm{D}\end{array}$ & 31.10 & $3 d^{3} 4 s 4 p{ }^{4} P .{ }^{3} P ;{ }^{4} D$ & 1.398 & \\
\hline
\end{tabular}


Table 5 (continued)

\begin{tabular}{|c|c|c|c|c|c|c|c|c|}
\hline$J$-value & \multirow{2}{*}{$\begin{array}{c}E_{o b s} \\
\left(\mathrm{~cm}^{-1}\right) \\
{[8,27]} \\
101170.469\end{array}$} & \multirow{2}{*}{$\begin{array}{c}\begin{array}{c}E_{\text {calc }} \\
\left(\mathrm{cm}^{-1}\right)\end{array} \\
101298\end{array}$} & \multicolumn{2}{|c|}{$\begin{array}{l}\text { Largest eigenvalue component } \\
(\%)\end{array}$} & \multicolumn{2}{|c|}{$\begin{array}{c}\text { Next largest eigenvalue component } \\
(\%)\end{array}$} & \multirow{2}{*}{$\begin{array}{r}\text { Calc. } g_{J} \\
0.764\end{array}$} & \multirow{2}{*}{$\begin{array}{c}\text { Obs. } g_{J} \\
{[5]} \\
\end{array}$} \\
\hline & & & 54.81 & $\begin{array}{l}3 d^{3} 4 s 4 p \\
{ }^{2} \mathrm{H} \cdot{ }^{3} \mathrm{P} ;{ }^{4} \mathrm{H}\end{array}$ & 24.28 & $3 d^{3} 4 s 4 p^{2} G \cdot{ }^{3} P ;{ }^{2} G$ & & \\
\hline & 101864.180 & 102024 & 32.46 & $\begin{array}{l}3 \mathrm{~d}^{3} 4 \mathrm{~s} 4 \mathrm{p} \\
{ }^{2} \mathrm{G} \cdot{ }^{3} \mathrm{P} ;{ }^{2} \mathrm{~F}\end{array}$ & 28.50 & $3 d^{3} 4 s 4 p^{2} G \cdot{ }^{3} P ;{ }^{2} G$ & 0.951 & \\
\hline & 102121.992 & 102264 & 33.30 & $\begin{array}{l}3 d^{3} 4 s 4 p \\
{ }^{2} \mathrm{G} \cdot{ }^{3} \mathrm{P} ;{ }^{2} \mathrm{~F}\end{array}$ & 26.94 & $3 d^{3} 4 s 4 p^{2} G \cdot{ }^{3} P ;{ }^{2} G$ & 1.045 & \\
\hline & 102492.539 & 102519 & 37.84 & $\begin{array}{l}3 \mathrm{~d}^{3} 4 \mathrm{~s} 4 \mathrm{p} \\
{ }^{2} \mathrm{D} \cdot{ }^{3} \mathrm{P} ;{ }^{4} \mathrm{~F}\end{array}$ & 11.96 & $3 d^{3} 4 s$ p ${ }^{2}$ G. ${ }^{3} \mathrm{P} ;{ }^{4} \mathrm{~F}$ & 1.233 & \\
\hline & \multirow[t]{2}{*}{102831.602} & 102909 & 42.89 & $\begin{array}{l}3 d^{3} 4 s 4 p \\
{ }^{2} \mathrm{P} \cdot{ }^{3} \mathrm{P} ;{ }^{4} \mathrm{D}\end{array}$ & 41.14 & $3 d^{3} 4 s 4 p{ }^{4} P .{ }^{3} P ;{ }^{4} D$ & 1.404 & \\
\hline & & 103097 & 74.74 & $\begin{array}{l}3 d^{3} 4 s 4 p \\
{ }^{4} \mathrm{~F} .{ }^{1} \mathrm{P} ;{ }^{4} \mathrm{G}\end{array}$ & 7.91 & $3 d^{3} 4 s 4 \mathrm{p}^{2} \mathrm{G} \cdot{ }^{3} \mathrm{P} ;{ }^{4} \mathrm{G}$ & 0.986 & \\
\hline & \multirow[t]{2}{*}{104680.789} & 104455 & 58.26 & $\begin{array}{l}3 d^{3} 4 s 4 p \\
2 D \cdot{ }^{3} P ;{ }^{4} D\end{array}$ & 15.94 & $3 d^{3} 4 s$ pp ${ }^{4} F \cdot{ }^{1} \mathrm{P} ;{ }^{4} \mathrm{D}$ & 1.427 & \\
\hline & & 105005 & 40.42 & $\begin{array}{l}3 \mathrm{~d}^{3} 4 \mathrm{~s} 4 \mathrm{p} \\
{ }^{4} \mathrm{~F} .{ }^{1} \mathrm{P} ;{ }^{4} \mathrm{D}\end{array}$ & 18.89 & $3 \mathrm{~d}^{3} 4 \mathrm{~s} 4 \mathrm{p}^{2} \mathrm{D} \cdot{ }^{3} \mathrm{P} ;{ }^{4} \mathrm{D}$ & 1.411 & \\
\hline & 105173.406 & 105172 & 49.84 & $3 d^{4} 4 f^{5} D ;{ }^{6} P$ & 21.34 & $3 d^{4} 4 f^{5} D ;{ }^{6} D$ & 1.469 & \\
\hline & 105197.312 & 105186 & 59.61 & $3 d^{4} 4 f^{5} D ;{ }^{6} H$ & 13.65 & $3 d^{4} 4 f^{5} D ;{ }^{4} H$ & 0.991 & \\
\hline & 105206.719 & 105267 & 68.35 & $3 d^{4} 4 f^{5} D ;{ }^{4} H$ & 12.22 & $3 d^{4} 4 f^{5} D ;{ }^{6} H$ & 0.795 & \\
\hline & \multirow[t]{2}{*}{105282.625} & 105302 & 41.16 & $3 d^{4} 4 f^{5} D ;{ }^{4} F$ & 18.76 & $3 d^{3} 4 s 4 p^{4} F .{ }^{1} \mathrm{P} ;{ }^{4} \mathrm{~F}$ & 1.188 & \\
\hline & & 105402 & 41.15 & $3 d^{4} 4 f^{5} \mathrm{D} ;{ }^{6} \mathrm{~F}$ & 19.66 & $3 d^{4} 4 f^{5} D ;{ }^{6} P$ & 1.419 & \\
\hline & 105419.961 & 105573 & 50.15 & $3 d^{4} 4 f^{5} D ;{ }^{6} G$ & 29.98 & $3 d^{4} 4 f^{5} D ;{ }^{6} D$ & 1.330 & \\
\hline & \multirow{2}{*}{105507.531} & 105627 & 71.19 & $3 d^{4} 4 f^{5} D ;{ }^{4} D$ & 7.85 & $3 d^{3} 4 s 4 p^{4} F .{ }^{1} \mathrm{P} ;{ }^{4} \mathrm{~F}$ & 1.431 & \\
\hline & & 105769 & 52.33 & $3 d^{4} 4 f^{5} D ;{ }^{6} F$ & 22.48 & $3 d^{3} 4 s 4 p^{5} D ;{ }^{6} D$ & 1.388 & \\
\hline & 105724.656 & 105821 & 72.65 & $3 d^{4} 4 f^{5} D ;{ }^{4} G$ & 11.42 & $\begin{array}{c}3 \mathrm{~d}^{3} 4 \mathrm{~s} 4 \mathrm{p}^{2} \mathrm{H} \\
{ }^{3} \mathrm{P} ;{ }^{4} \mathrm{G}\end{array}$ & 1.002 & \\
\hline & 105985.555 & 106114 & 60.85 & $\begin{array}{l}3 \mathrm{~d}^{3} 4 \mathrm{~s} 4 \mathrm{p} \\
{ }^{2} \mathrm{H} \cdot{ }^{3} \mathrm{P} ;{ }^{4} \mathrm{G}\end{array}$ & 11.82 & $3 d^{4} 4 f^{5} D ;{ }^{4} F$ & 1.052 & \\
\hline & 106779.250 & 106269 & 31.23 & $3 d^{4} 4 f^{5} D ;{ }^{4} F$ & 27.67 & $3 d^{3} 4 s 4 p{ }^{4} F .{ }^{1} \mathrm{P} ;{ }^{4} \mathrm{~F}$ & 1.166 & \\
\hline & 107153.039 & 107281 & 65.84 & $\begin{array}{l}3 d^{3} 4 s 4 p \\
2{ }^{3} \cdot{ }^{3} ;{ }^{2} \mathrm{~F}\end{array}$ & 20.30 & $3 d^{3} 4 s 4 p^{2}$ D. ${ }^{3} \mathrm{P} ;{ }^{2} \mathrm{~F}$ & 1.143 & \\
\hline & \multirow[t]{3}{*}{107918.547} & 107604 & 83.07 & $\begin{array}{l}3 \mathrm{~d}^{3} 4 \mathrm{~s} 4 \mathrm{p} \\
{ }^{2} \mathrm{H} \cdot{ }^{3} \mathrm{P} ;{ }^{2} \mathrm{G}\end{array}$ & 9.91 & $3 d^{3} 4 s 4 p{ }^{2} F \cdot{ }^{3} \mathrm{P} ;{ }^{2} \mathrm{G}$ & 0.893 & \\
\hline & & 109048 & 88.75 & $3 d^{4} 5 p^{3} \mathrm{H} ;{ }^{4} \mathrm{H}$ & 8.13 & $3 d^{4} 5 p^{3} H ;{ }^{2} G$ & 0.688 & \\
\hline & & 109745 & 80.70 & $3 d^{4} 5 p^{3} H ;{ }^{2} G$ & 9.01 & $3 \mathrm{~d}^{4} 5 \mathrm{p}^{3} \mathrm{H} ;{ }^{4} \mathrm{H}$ & 0.875 & \\
\hline & 109812.039 & 109796 & 94.66 & $3 d^{4} 5 p^{5} \mathrm{D} ;{ }^{6} \mathrm{~F}$ & 2.62 & $3 d^{4} 6 p^{5} D ;{ }^{4} F$ & 1.395 & \\
\hline & 110097.094 & 110034 & 89.90 & $3 d^{4} 5 p^{5} D ;{ }^{6} P$ & 7.77 & $3 d^{4} 6 p^{5} D ;{ }^{6} D$ & 1.700 & \\
\hline & \multirow[t]{2}{*}{110272.117} & 110134 & 83.10 & $3 d^{4} 5 p^{5} D ;{ }^{6} D$ & 7.02 & $3 \mathrm{~d}^{4} 6 \mathrm{p}^{5} \mathrm{D} ;{ }^{6} \mathrm{P}$ & 1.568 & \\
\hline & & 110400 & 81.90 & $3 d^{4} 5 p^{3} \mathrm{H} ;{ }^{4} \mathrm{G}$ & 4.37 & $3 d^{4} 5 p^{3} \mathrm{H} ;{ }^{2} \mathrm{G}$ & 0.990 & \\
\hline & 110471.281 & 110506 & 80.88 & $3 d^{4} 5 p^{5} D ;{ }^{4} F$ & 7.32 & $3 d^{4} 6 p^{5} D ;{ }^{6} D$ & 1.265 & \\
\hline & \multirow[t]{14}{*}{111269.164} & 111265 & 35.91 & $3 d^{4} 5 p^{3} p ;{ }^{4} D$ & 24.20 & $3 d^{4} 5 p^{3} p ;{ }^{4} D$ & 1.426 & \\
\hline & & 111323 & 70.12 & $3 d^{4} 5 p^{5} D ;{ }^{4} D$ & 8.89 & $3 d^{4} 5 p^{3} F ;{ }^{4} D$ & 1.429 & \\
\hline & & 111490 & 66.61 & $3 d^{4} 5 p{ }^{3} F ;{ }^{4} G$ & 20.00 & $3 d^{4} 5 p^{3} F ;{ }^{4} G$ & 0.991 & \\
\hline & & 111999 & 39.93 & $3 d^{4} 5 p^{3} F ;{ }^{4} F$ & 10.72 & $3 d^{4} 5 p^{3} F ;{ }^{4} F$ & 1.256 & \\
\hline & & 112107 & 29.55 & $3 d^{4} 5 p^{3} F ;{ }^{2} G$ & 15.72 & $3 d^{4} 5 p^{3} F ;{ }^{2} F$ & 1.073 & \\
\hline & & 112228 & 33.16 & $3 d^{4} 5 p^{3} F ;{ }^{2} G$ & 23.71 & $3 d^{4} 5 p^{3} F ;{ }^{4} F$ & 1.087 & \\
\hline & & 112404 & 36.64 & $3 d^{4} 5 p^{3} F ;{ }^{2} F$ & 27.34 & $3 d^{4} 5 p^{3} F ;{ }^{4} D$ & 1.269 & \\
\hline & & 113366 & 60.04 & $3 \mathrm{~d}^{4} 4 \mathrm{p}{ }^{1} \mathrm{D} ;{ }^{2} \mathrm{~F}$ & 17.00 & $3 d^{4} 4 p{ }^{1} D ;{ }^{2} F$ & 1.143 & \\
\hline & & 113643 & 93.24 & $3 d^{4} 5 p^{3} \mathrm{G} ;{ }^{4} \mathrm{H}$ & 2.29 & $3 d^{4} 5 p^{3} G ;{ }^{2} G$ & 0.680 & \\
\hline & & 114056 & 38.23 & $3 d^{4} 5 p^{3} G ;{ }^{4} F$ & 29.72 & $3 d^{4} 5 p^{3} G ;{ }^{4} G$ & 1.153 & \\
\hline & & 114275 & 59.02 & $3 d^{4} 5 p^{3} G ;{ }^{4} G$ & 16.66 & $3 d^{3} 4 s 4 p^{2} F \cdot{ }^{3} P ;{ }^{4} F$ & 1.056 & \\
\hline & & 114542 & 74.80 & $3 d^{4} 5 p^{3} G ;{ }^{2} G$ & 6.72 & $3 d^{3} 4 s 4 p^{2} F \cdot{ }^{3} P ;{ }^{4} F$ & 0.926 & \\
\hline & & 114765 & 70.27 & $3 d^{4} 5 p^{3} G ;{ }^{2} F$ & 7.20 & $3 d^{4} 4 p{ }^{1} D ;{ }^{2} F$ & 1.125 & \\
\hline & & 115149 & 42.79 & $\begin{array}{l}3 d^{3} 4 s 4 p \\
{ }^{2} \mathrm{~F}^{3} \mathrm{P} ;{ }^{4} \mathrm{~F}\end{array}$ & 34.90 & $3 d^{4} 5 p^{3} G ;{ }^{4} F$ & 1.233 & \\
\hline & 115249.250 & 115255 & 37.15 & $3 d^{4} 5 f^{5} D ;{ }^{6} P$ & 27.08 & $3 d^{4} 5 f^{5} D ;{ }^{6} D$ & 1.415 & \\
\hline & 115309.094 & 115287 & 48.60 & $3 d^{4} 5 f^{5} D ;{ }^{6} H$ & 14.72 & $3 d^{4} 5 f^{5} D ;{ }^{4} H$ & 1.018 & \\
\hline & 115398.391 & 115342 & 69.96 & $3 d^{4} 5 f^{5} D ;{ }^{4} H$ & 15.21 & $3 d^{4} 5 f^{5} D ;{ }^{6} H$ & 0.757 & \\
\hline & \multirow[t]{2}{*}{115447.578} & 115419 & 30.10 & $3 d^{4} 5 f^{5} D ;{ }^{4} D$ & 24.36 & $3 d^{4} 5 f^{5} D ;{ }^{6} F$ & 1.336 & \\
\hline & & 115474 & 29.61 & $3 d^{4} 5 f^{5} D ;{ }^{4} D$ & 16.25 & $3 d^{4} 5 f^{5} D ;{ }^{6} P$ & 1.328 & \\
\hline & 115591.148 & 115599 & 44.20 & $3 d^{4} 5 f^{5} D ;{ }^{6} G$ & 22.86 & $3 d^{4} 5 f^{5} D ;{ }^{6} D$ & 1.349 & \\
\hline & 115627.719 & 115649 & 55.05 & $3 d^{4} 5 f^{5} D ;{ }^{4} G$ & 20.48 & $3 d^{4} 5 f^{5} D ;{ }^{4} D$ & 1.140 & \\
\hline & 115797.203 & 115789 & 44.59 & $3 d^{4} 5 f^{5} D ;{ }^{6} F$ & 35.91 & $3 d^{4} 5 f^{5} D ;{ }^{6} D$ & 1.453 & \\
\hline & \multirow[t]{5}{*}{115882.164} & 115889 & 55.63 & $3 d^{4} 5 f^{5} D ;{ }^{4} F$ & 24.87 & $3 d^{4} 5 f^{5} D ;{ }^{4} G$ & 1.189 & \\
\hline & & 116735 & 93.40 & $\begin{array}{l}3 d^{3} 4 s 4 p \\
{ }^{2} F^{3} P ;{ }^{4} G\end{array}$ & 3.71 & $\begin{array}{c}3 d^{3} 4 s 4 p^{2} \mathrm{H} \\
{ }^{3} \mathrm{P} ;{ }^{4} \mathrm{G}\end{array}$ & 0.987 & \\
\hline & & 117045 & 64.44 & $\begin{array}{l}3 d^{3} 4 s 4 p \\
2 F^{3} P ;{ }^{4} D\end{array}$ & 22.29 & $3 d^{3} 4 s$ p ${ }^{4} P .{ }^{1} \mathrm{P} ;{ }^{4} \mathrm{D}$ & 1.428 & \\
\hline & & 117526 & 41.94 & $\begin{array}{l}3 d^{3} 4 s 4 p \\
{ }^{2} G .{ }^{1} P ;{ }^{2} G\end{array}$ & 26.01 & $3 d^{4} 5 p{ }^{1} G ;{ }^{2} G$ & 0.896 & \\
\hline & & 117964 & 43.43 & $3 d^{4} 5 p^{1} G ;{ }^{2} F$ & 24.57 & $3 d^{4} 5 p{ }^{1} G ;{ }^{2} F$ & 1.136 & \\
\hline
\end{tabular}


Table 5 (continued)

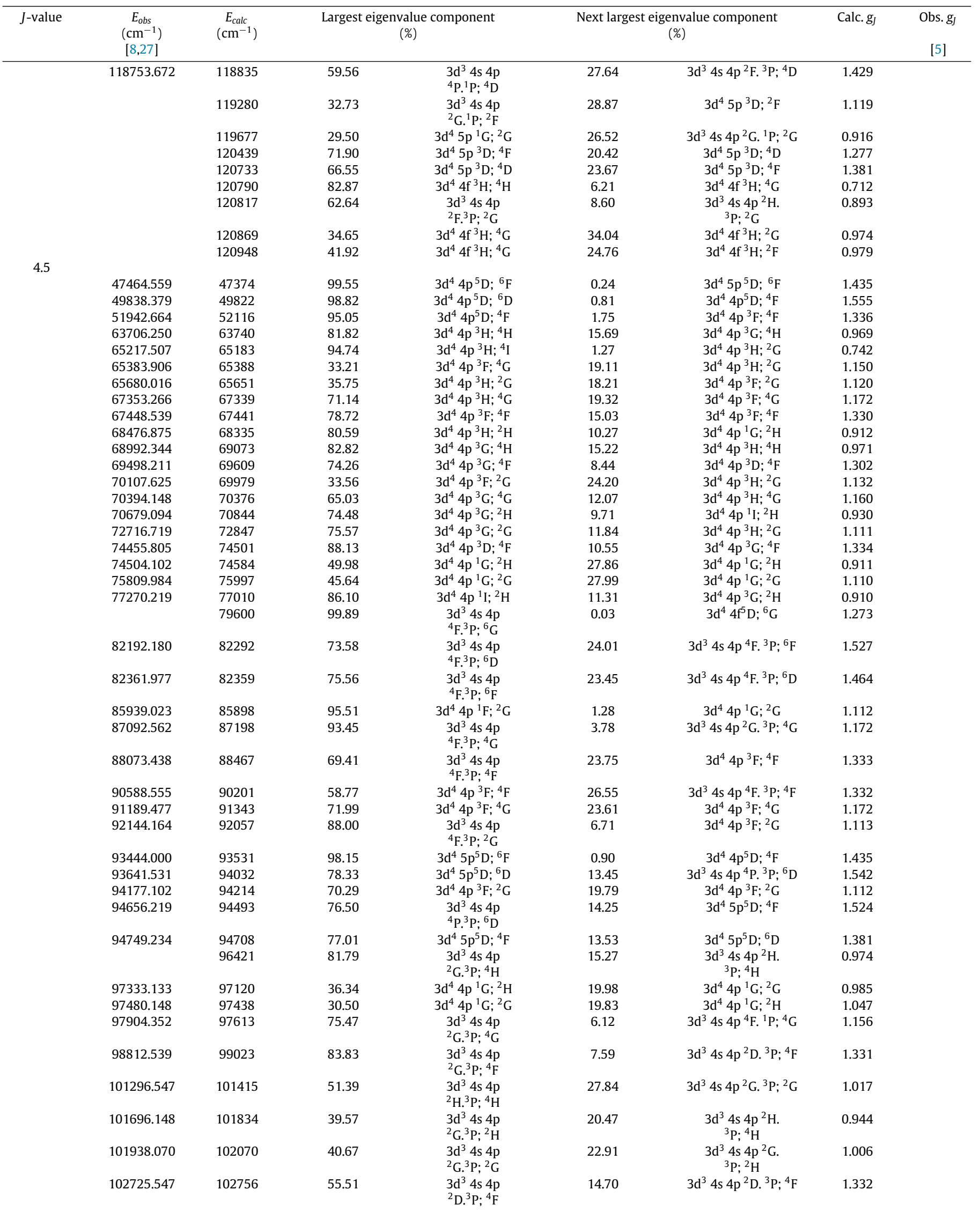


Table 5 (continued)

\begin{tabular}{|c|c|c|c|c|c|c|c|c|}
\hline \multirow[t]{2}{*}{$J$-value } & \multirow{2}{*}{$\begin{array}{c}E_{o b s} \\
\left(\mathrm{~cm}^{-1}\right) \\
{[8,27]} \\
103199.812\end{array}$} & \multirow{2}{*}{$\begin{array}{c}\begin{array}{c}E_{\text {calc }} \\
\left(\mathrm{cm}^{-1}\right)\end{array} \\
103055\end{array}$} & \multicolumn{2}{|c|}{$\begin{array}{l}\text { Largest eigenvalue component } \\
\qquad(\%)\end{array}$} & \multicolumn{2}{|c|}{$\begin{array}{c}\text { Next largest eigenvalue component } \\
(\%)\end{array}$} & \multirow{2}{*}{$\begin{array}{r}\text { Calc. } g_{J} \\
0.755\end{array}$} & \multirow{2}{*}{$\begin{array}{c}\text { Obs. } g_{J} \\
{[5]} \\
\end{array}$} \\
\hline & & & 84.89 & $\begin{array}{l}3 \mathrm{~d}^{3} 4 \mathrm{~s} 4 \mathrm{p} \\
{ }^{2} \mathrm{H} .{ }^{3} \mathrm{P} ;{ }^{4} \mathrm{I}\end{array}$ & 10.96 & $\begin{array}{c}3 d^{3} 4 s 4 p^{2} G \\
{ }^{3} \mathrm{P} ;{ }^{2} \mathrm{H}\end{array}$ & & \\
\hline & 105203.461 & 103361 & 74.19 & $\begin{array}{l}3 d^{3} 4 s 4 p \\
{ }^{4} \mathrm{~F} .{ }^{1} \mathrm{P} ;{ }^{4} \mathrm{G}\end{array}$ & 8.85 & $3 d^{4} 4 f^{5} D ;{ }^{4} G$ & 1.171 & \\
\hline & 105263.438 & 105237 & 74.73 & $3 d^{4} 4 f^{5} D ;{ }^{6} H$ & 14.41 & $3 d^{4} 4 f^{5} D ;{ }^{4} H$ & 1.083 & \\
\hline & 105398.156 & 105361 & 46.10 & $3 d^{4} 4 f^{5} D ;{ }^{6} D$ & 22.39 & $3 d^{4} 4 f^{5} D ;{ }^{6} F$ & 1.432 & \\
\hline & 105406.898 & 105385 & 78.57 & $3 d^{4} 4 f^{5} D ;{ }^{4} H$ & 9.54 & $3 \mathrm{~d}^{4} 4 \mathrm{f}^{5} \mathrm{D} ;{ }^{6} \mathrm{H}$ & 1.029 & \\
\hline & & 105471 & 51.45 & $3 d^{4} 4 f^{5} D ;{ }^{4} F$ & 16.65 & $3 d^{3} 4 s 4 p^{4} F \cdot{ }^{1} \mathrm{P} ;{ }^{4} \mathrm{~F}$ & 1.330 & \\
\hline & 105623.547 & 105590 & 44.45 & $3 d^{4} 4 f^{5} D ;{ }^{6} G$ & 34.15 & $3 d^{4} 4 f^{5} D ;{ }^{6} D$ & 1.379 & \\
\hline & & 105777 & 80.76 & $\begin{array}{l}3 \mathrm{~d}^{3} 4 \mathrm{~s} 4 \mathrm{p} \\
{ }^{2} \mathrm{H} \cdot{ }^{3} \mathrm{P} ;{ }^{2} \mathrm{H}\end{array}$ & 13.69 & $3 \mathrm{~d}^{4} 4 \mathrm{f}^{2} \mathrm{G} \cdot{ }^{3} \mathrm{P} ;{ }^{2} \mathrm{H}$ & 0.914 & \\
\hline & 105789.961 & 105809 & 51.78 & $3 d^{4} 4 f^{5} D ;{ }^{6} F$ & 36.60 & $3 d^{4} 4 f^{5} D ;{ }^{6} G$ & 1.383 & \\
\hline & 106045.469 & 105925 & 60.91 & $3 d^{4} 4 f^{5} D ;{ }^{4} G$ & 24.66 & $\begin{array}{c}3 d^{3} 4 s 4 p^{2} \mathrm{H} \\
{ }^{3} \mathrm{P} ;{ }^{4} \mathrm{G}\end{array}$ & 1.175 & \\
\hline & 106165.352 & 106159 & 57.52 & $\begin{array}{l}3 \mathrm{~d}^{3} 4 \mathrm{~s} 4 \mathrm{p} \\
{ }^{2} \mathrm{H} \cdot{ }^{3} \mathrm{P} ;{ }^{4} \mathrm{G}\end{array}$ & 17.91 & $3 d^{4} 4 f^{5} D ;{ }^{4} G$ & 1.183 & \\
\hline & 106791.977 & 106452 & 47.99 & $\begin{array}{l}3 d^{3} 4 s 4 p \\
{ }^{4} \mathrm{~F} .{ }^{1} \mathrm{P} ;{ }^{4} \mathrm{~F}\end{array}$ & 28.49 & $3 d^{4} 4 f^{5} D ;{ }^{4} F$ & 1.318 & \\
\hline & 107739.234 & 107403 & 82.87 & $\begin{array}{l}3 \mathrm{~d}^{3} 4 \mathrm{~s} 4 \mathrm{p} \\
{ }^{2} \mathrm{H} \cdot{ }^{3} \mathrm{P} ;{ }^{2} \mathrm{G}\end{array}$ & 9.12 & $3 \mathrm{~d}^{3} 4 \mathrm{~s} 4 \mathrm{p}^{2} \mathrm{~F} \cdot{ }^{3} \mathrm{P} ;{ }^{2} \mathrm{G}$ & 1.111 & \\
\hline & & 109021 & 69.74 & $3 d^{4} 5 p^{3} \mathrm{H} ;{ }^{4} \mathrm{I}$ & 25.45 & $3 d^{4} 5 p^{3} \mathrm{H} ;{ }^{4} \mathrm{H}$ & 0.798 & \\
\hline & & 109227 & 68.61 & $3 d^{4} 5 p^{3} \mathrm{H} ;{ }^{4} \mathrm{H}$ & 24.53 & $3 d^{4} 5 p^{3} \mathrm{H} ;{ }^{4} \mathrm{I}$ & 0.915 & \\
\hline & & 109843 & 51.57 & $3 d^{4} 5 p^{3} H ;{ }^{2} G$ & 27.54 & $3 d^{4} 5 p^{3} \mathrm{H} ;{ }^{2} \mathrm{H}$ & 1.046 & \\
\hline & & 110007 & 92.77 & $3 d^{4} 6 p^{5} D ;{ }^{6} F$ & 3.67 & $3 \mathrm{~d}^{4} 6 \mathrm{p}^{5} \mathrm{D} ;{ }^{4} \mathrm{~F}$ & 1.433 & \\
\hline & & 110083 & 61.32 & $3 d^{4} 5 p^{3} \mathrm{H} ;{ }^{2} \mathrm{H}$ & 30.38 & $3 d^{4} 5 p^{3} H ;{ }^{2} G$ & 0.974 & \\
\hline & & 110278 & 78.11 & $3 d^{4} 6 p^{5} D ;{ }^{6} D$ & 15.04 & $3 \mathrm{~d}^{4} 6 \mathrm{p}^{5} \mathrm{D} ;{ }^{4} \mathrm{~F}$ & 1.513 & \\
\hline & & 110451 & 78.93 & $3 d^{4} 5 p^{3} \mathrm{H} ;{ }^{4} \mathrm{G}$ & 7.71 & $3 d^{4} 5 p^{3} H ;{ }^{2} G$ & 1.162 & \\
\hline & 110665.477 & 110676 & 74.28 & $3 d^{4} 6 p^{5} D ;{ }^{4} F$ & 18.78 & $3 d^{4} 6 p^{5} D ;{ }^{6} D$ & 1.376 & \\
\hline & & 111628 & 61.25 & $3 d^{4} 5 p^{3} F ;{ }^{4} G$ & 18.06 & $3 d^{4} 5 p^{3} F ;{ }^{4} G$ & 1.176 & \\
\hline & & 112055 & 33.75 & $3 d^{4} 5 p^{3} F ;{ }^{4} F$ & 24.58 & $3 d^{4} 5 p^{3} F ;{ }^{2} G$ & 1.222 & \\
\hline & & 112309 & 37.97 & $3 d^{4} 5 p^{3} F ;{ }^{2} G$ & 34.08 & $3 d^{4} 5 p^{3} F ;{ }^{4} F$ & 1.211 & \\
\hline & & 113807 & 92.60 & $3 d^{4} 5 p^{3} \mathrm{G} ;{ }^{4} \mathrm{H}$ & 1.42 & $3 d^{4} 5 p^{3} G ;{ }^{4} G$ & 0.977 & \\
\hline & & 114120 & 47.57 & $3 d^{4} 5 p^{3} G ;{ }^{4} F$ & 27.81 & $3 d^{3} 4 s 4 p^{2} F \cdot{ }^{3} P ;{ }^{4} F$ & 1.297 & \\
\hline & & 114334 & 57.88 & $3 d^{4} 5 p^{3} G ;{ }^{4} G$ & 16.71 & $3 d^{4} 5 p^{3} G ;{ }^{2} \mathrm{H}$ & 1.142 & \\
\hline & & 114540 & 75.05 & $3 d^{4} 5 p^{3} G ;{ }^{2} \mathrm{H}$ & 16.87 & $3 d^{4} 5 p^{3} G ;{ }^{4} G$ & 0.970 & \\
\hline & & 114726 & 77.87 & $3 d^{4} 5 p^{3} G ;{ }^{2} G$ & 10.62 & $3 d^{3} 4 s 4 p^{2} F \cdot{ }^{3} P ;{ }^{4} F$ & 1.131 & \\
\hline & & 115191 & 44.38 & $\begin{array}{l}3 \mathrm{~d}^{3} 4 \mathrm{~s} 4 \mathrm{p} \\
{ }^{2} \mathrm{~F} \cdot{ }^{3} \mathrm{P} ;{ }^{4} \mathrm{~F}\end{array}$ & 36.02 & $3 d^{4} 5 p^{3} G ;{ }^{4} F$ & 1.318 & \\
\hline & 115288.750 & 115314 & 56.30 & $3 d^{4} 5 f^{5} D ;{ }^{6} H$ & 17.06 & $3 d^{4} 5 f^{5} D ;{ }^{6} G$ & 1.134 & \\
\hline & 115393.398 & 115414 & 41.43 & $3 d^{4} 5 f^{5} D ;{ }^{6} D$ & 31.93 & $3 d^{4} 5 f^{5} D ;{ }^{6} F$ & 1.402 & \\
\hline & 115430.406 & 115452 & 71.62 & $3 d^{4} 5 f^{5} D ;{ }^{4} H$ & 13.82 & $3 d^{4} 5 f^{5} D ;{ }^{6} H$ & 1.020 & \\
\hline & 115592.359 & 115597 & 46.25 & $3 d^{4} 5 f^{5} D ;{ }^{6} G$ & 36.21 & $3 d^{4} 5 f^{5} D ;{ }^{6} D$ & 1.365 & \\
\hline & 115672.797 & 115695 & 40.75 & $3 d^{4} 5 f^{5} D ;{ }^{4} G$ & 40.59 & $3 d^{4} 5 f^{5} D ;{ }^{4} F$ & 1.234 & \\
\hline & 115824.430 & 115812 & 53.73 & $3 d^{4} 5 f^{5} D ;{ }^{6} F$ & 28.62 & $3 d^{4} 5 f^{5} D ;{ }^{6} G$ & 1.402 & \\
\hline & 115927.320 & 115933 & 47.46 & $3 d^{4} 5 f^{5} D ;{ }^{4} F$ & 43.38 & $3 d^{4} 5 f^{5} D ;{ }^{4} G$ & 1.254 & \\
\hline & & 116500 & 50.93 & $3 d^{4} 5 p{ }^{1} \mathrm{I} ;{ }^{2} \mathrm{H}$ & 37.89 & $\begin{array}{c}3 \mathrm{~d}^{3} 4 \mathrm{~s} 4 \mathrm{p}^{2} \mathrm{G} \\
{ }^{1} \mathrm{P} ;{ }^{2} \mathrm{H}\end{array}$ & 0.910 & \\
\hline & & 116809 & 93.62 & $\begin{array}{l}3 d^{3} 4 s 4 p \\
{ }^{2} \mathrm{~F} \cdot{ }^{3} \mathrm{P} ;{ }^{4} \mathrm{G}\end{array}$ & 3.66 & $\begin{array}{c}3 d^{3} 4 s 4 p^{2} \mathrm{H} \\
{ }^{3} \mathrm{P} ;{ }^{4} \mathrm{G}\end{array}$ & 1.174 & \\
\hline & & 117636 & 39.66 & $\begin{array}{l}3 d^{3} 4 s 4 p \\
{ }^{2} \mathrm{G} \cdot{ }^{1} \mathrm{P} ;{ }^{2} \mathrm{G}\end{array}$ & 29.94 & $3 d^{4} 5 p{ }^{1} G ;{ }^{2} G$ & 1.108 & \\
\hline & & 118207 & 57.30 & $3 \mathrm{~d}^{4} 5 \mathrm{p}^{1} \mathrm{G} ;{ }^{2} \mathrm{H}$ & 31.36 & $3 d^{4} 5 p{ }^{1} G ;{ }^{2} H$ & 0.913 & \\
\hline & & 119484 & 44.61 & $\begin{array}{l}3 \mathrm{~d}^{3} 4 \mathrm{~s} 4 \mathrm{p} \\
{ }^{2} \mathrm{G} \cdot{ }^{1} \mathrm{P} ;{ }^{2} \mathrm{H}\end{array}$ & 32.14 & $3 d^{4} 5 p{ }^{1} \mathrm{I} ;{ }^{2} \mathrm{H}$ & 0.921 & \\
\hline & & 119848 & 29.56 & $\begin{array}{l}3 d^{3} 4 s 4 p \\
{ }^{2} \mathrm{G} .{ }^{1} \mathrm{P} ;{ }^{2} \mathrm{G}\end{array}$ & 27.72 & $3 d^{4} 5 p{ }^{1} G ;{ }^{2} G$ & 1.092 & \\
\hline & & 120614 & 98.24 & $3 d^{4} 5 p^{3} D ;{ }^{4} F$ & 0.35 & $3 d^{4} 5 p^{3} G ;{ }^{4} F$ & 1.332 & \\
\hline & & 120642 & 67.19 & $3 \mathrm{~d}^{4} 4 \mathrm{f}^{3} \mathrm{H} ;{ }^{2} \mathrm{H}$ & 10.50 & $\begin{array}{c}3 d^{3} 4 s 4 p^{2} \mathrm{H} \\
{ }^{1} \mathrm{P} ;{ }^{2} \mathrm{H}\end{array}$ & 0.923 & \\
\hline & & 120750 & 80.84 & $3 \mathrm{~d}^{4} 4 \mathrm{f}^{3} \mathrm{H} ;{ }^{4} \mathrm{I}$ & 15.19 & $3 \mathrm{~d}^{4} 4 \mathrm{f}^{3} \mathrm{H} ;{ }^{4} \mathrm{H}$ & 0.775 & \\
\hline & & 120865 & 69.06 & $3 \mathrm{~d}^{4} 4 \mathrm{f}^{3} \mathrm{H} ;{ }^{4} \mathrm{H}$ & 13.76 & $3 \mathrm{~d}^{4} 4 \mathrm{f}^{3} \mathrm{H} ;{ }^{4} \mathrm{I}$ & 0.961 & \\
\hline & & 120953 & 43.13 & $3 \mathrm{~d}^{4} 4 \mathrm{f}^{3} \mathrm{H} ;{ }^{4} \mathrm{G}$ & 21.79 & $3 \mathrm{~d}^{4} 4 \mathrm{f}^{3} \mathrm{H} ;{ }^{2} \mathrm{G}$ & 1.143 & \\
\hline \multicolumn{9}{|l|}{5.5} \\
\hline & 47751.602 & 47627 & 99.62 & $3 d^{4} 4 p^{5} D ;{ }^{6} F$ & 0.24 & $3 d^{4} 5 p^{5} D ;{ }^{6} F$ & 1.456 & \\
\hline & 63848.699 & 63870 & 82.32 & $3 d^{4} 4 p^{3} \mathrm{H} ;{ }^{4} \mathrm{H}$ & 14.99 & $3 d^{4} 4 p^{3} G ;{ }^{4} \mathrm{H}$ & 1.131 & \\
\hline & 65419.516 & 65362 & 97.57 & $3 d^{4} 4 p^{3} \mathrm{H} ;{ }^{4} \mathrm{I}$ & 1.72 & $3 \mathrm{~d}^{4} 4 \mathrm{p}^{3} \mathrm{H} ;{ }^{4} \mathrm{H}$ & 0.968 & \\
\hline & 65709.445 & 65744 & 45.69 & $3 d^{4} 4 p{ }^{3} F ;{ }^{4} G$ & 18.79 & $3 d^{4} 4 p^{3} F ;{ }^{4} G$ & 1.271 & \\
\hline & 67369.070 & 67356 & 67.97 & $3 d^{4} 4 p^{3} \mathrm{H} ;{ }^{4} \mathrm{G}$ & 22.85 & $3 d^{4} 4 p^{3} F ;{ }^{4} G$ & 1.271 & \\
\hline & 67506.086 & 67689 & 91.76 & $3 \mathrm{~d}^{4} 4 \mathrm{p}^{3} \mathrm{H} ;{ }^{2} \mathrm{I}$ & 6.03 & $3 d^{4} 4 p^{1} \mathrm{I} ;{ }^{2} \mathrm{I}$ & 0.927 & \\
\hline & 68737.773 & 68566 & 83.15 & $3 d^{4} 4 p^{3} \mathrm{H} ;{ }^{2} \mathrm{H}$ & 8.79 & $3 d^{4} 4 p{ }^{1} G ;{ }^{2} \mathrm{H}$ & 1.092 & \\
\hline & 69170.352 & 69237 & 83.41 & $3 d^{4} 4 p^{3} \mathrm{G} ;{ }^{4} \mathrm{H}$ & 14.46 & $3 \mathrm{~d}^{4} 4 \mathrm{p}^{3} \mathrm{H} ;{ }^{4} \mathrm{H}$ & 1.133 & \\
\hline
\end{tabular}


Table 5 (continued)

\begin{tabular}{|c|c|c|c|c|c|c|c|c|}
\hline$J$-value & \multirow{2}{*}{$\begin{array}{c}E_{o b s} \\
\left(\mathrm{~cm}^{-1}\right) \\
{[8,27]} \\
70398.805\end{array}$} & \multirow{2}{*}{$\begin{array}{c}E_{\text {calc }} \\
\left(\mathrm{cm}^{-1}\right)\end{array}$} & \multicolumn{2}{|c|}{$\begin{array}{l}\text { Largest eigenvalue component } \\
\text { (\%) }\end{array}$} & \multicolumn{2}{|c|}{$\begin{array}{c}\text { Next largest eigenvalue component } \\
(\%)\end{array}$} & \multirow{2}{*}{$\begin{array}{c}\text { Calc. } g_{J} \\
1.114\end{array}$} & \multirow{2}{*}{$\begin{array}{c}\text { Obs. } g_{J} \\
{[5]}\end{array}$} \\
\hline & & & 70.07 & $3 d^{4} 4 p^{3} G ;{ }^{2} H$ & 10.52 & $3 d^{4} 4 p^{1} \mathrm{I} ;{ }^{2} \mathrm{H}$ & & \\
\hline & 70879.727 & 71024 & 67.98 & $3 d^{4} 4 p^{3} G ;{ }^{4} G$ & 11.94 & $3 d^{4} 4 p^{3} H ;{ }^{4} G$ & 1.249 & \\
\hline & 74421.727 & 74286 & 86.40 & $3 \mathrm{~d}^{4} 4 \mathrm{p}{ }^{1} \mathrm{I} ;{ }^{2} \mathrm{I}$ & 5.25 & $3 d^{4} 4 p^{3} \mathrm{H} ;{ }^{2} \mathrm{I}$ & 0.937 & \\
\hline & 74707.391 & 74793 & 47.53 & $3 d^{4} 4 p^{1} G ;{ }^{2} \mathrm{H}$ & 25.38 & $3 d^{4} 4 p^{1} G ;{ }^{2} \mathrm{H}$ & 1.079 & \\
\hline & 77078.859 & 76811 & 84.75 & $3 \mathrm{~d}^{4} 4 \mathrm{p}^{1} \mathrm{I} ;{ }^{2} \mathrm{H}$ & 11.95 & $3 d^{4} 4 p^{3} G ;{ }^{2} \mathrm{H}$ & 1.090 & \\
\hline & & 79929 & 99.90 & $\begin{array}{l}3 d^{3} 4 s 4 p \\
{ }^{4} \mathrm{~F} \cdot{ }^{3} \mathrm{P} ;{ }^{6} \mathrm{G}\end{array}$ & 0.03 & $3 d^{4} 4 f^{5} D ;{ }^{6} G$ & 1.343 & \\
\hline & 82612.570 & 82613 & 99.43 & $\begin{array}{l}3 d^{3} 4 s 4 p \\
{ }^{4} \mathrm{~F} \cdot{ }^{3} \mathrm{P} ;{ }^{6} \mathrm{~F}\end{array}$ & 0.21 & $3 \mathrm{~d}^{3} 4 \mathrm{~s} 4 \mathrm{p}{ }^{4} \mathrm{~F} \cdot{ }^{3} \mathrm{P} ;{ }^{4} \mathrm{G}$ & 1.455 & \\
\hline & 87450.438 & 87560 & 93.29 & $\begin{array}{l}3 d^{3} 4 s 4 p \\
{ }^{4} \mathrm{~F} \cdot{ }^{3} \mathrm{P} ;{ }^{4} \mathrm{G}\end{array}$ & 3.74 & $3 \mathrm{~d}^{3} 4 \mathrm{~s} 4 \mathrm{p}^{2} \mathrm{G} \cdot{ }^{3} \mathrm{P} ;{ }^{4} \mathrm{G}$ & 1.274 & \\
\hline & 91291.969 & 91433 & 73.43 & $3 d^{4} 4 p^{3} F ;{ }^{4} G$ & 23.63 & $3 d^{4} 4 p^{3} F ;{ }^{4} G$ & 1.273 & \\
\hline & 93643.380 & 93799 & 99.46 & $3 d^{4} 5 p^{5} D ;{ }^{6} F$ & 0.24 & $3 d^{4} 4 p^{5} D ;{ }^{6} F$ & 1.456 & \\
\hline & & 96676 & 81.34 & $\begin{array}{l}3 d^{3} 4 s 4 p \\
{ }^{2} \mathrm{G} \cdot{ }^{3} \mathrm{P} ;{ }^{4} \mathrm{H}\end{array}$ & 15.55 & $\begin{array}{c}3 \mathrm{~d}^{3} 4 \mathrm{~s} 4 \mathrm{p}^{2} \mathrm{H} \\
{ }^{3} \mathrm{P} ;{ }^{4} \mathrm{H}\end{array}$ & 1.136 & \\
\hline & 97493.523 & 97499 & 56.25 & $3 d^{4} 4 p^{1} G ;{ }^{2} \mathrm{H}$ & 28.87 & $3 \mathrm{~d}^{4} 4 \mathrm{p}{ }^{1} \mathrm{G} ;{ }^{2} \mathrm{H}$ & 1.096 & \\
\hline & 97899.367 & 97781 & 81.44 & $\begin{array}{l}3 \mathrm{~d}^{3} 4 \mathrm{~s} 4 \mathrm{p} \\
{ }^{2} \mathrm{G} \cdot{ }^{3} \mathrm{P} ;{ }^{4} \mathrm{G}\end{array}$ & 5.98 & $3 \mathrm{~d}^{3} 4 \mathrm{~s} 4 \mathrm{p}{ }^{4} \mathrm{~F} .{ }^{1} \mathrm{P} ;{ }^{4} \mathrm{G}$ & 1.264 & \\
\hline & 101782.969 & 101718 & 83.17 & $\begin{array}{l}3 \mathrm{~d}^{3} 4 \mathrm{~s} 4 \mathrm{p} \\
{ }^{2} \mathrm{H} .{ }^{3} \mathrm{P} ;{ }^{4} \mathrm{H}\end{array}$ & 15.33 & $\begin{array}{c}3 \mathrm{~d}^{3} 4 \mathrm{~s} 4 \mathrm{p}^{2} \mathrm{G} \\
{ }^{3} \mathrm{P} ;{ }^{4} \mathrm{H}\end{array}$ & 1.134 & \\
\hline & 101932.234 & 102124 & 69.60 & $\begin{array}{l}3 d^{3} 4 s 4 p \\
{ }^{2} \mathrm{G} \cdot{ }^{3} \mathrm{P} ;{ }^{2} \mathrm{H}\end{array}$ & 13.48 & $\begin{array}{c}3 d^{3} 4 s 4 p^{2} \mathrm{H} \\
{ }^{3} \mathrm{P} ;{ }^{2} \mathrm{H}\end{array}$ & 1.084 & \\
\hline & & 103155 & 93.20 & $\begin{array}{l}3 \mathrm{~d}^{3} 4 \mathrm{~s} 4 \mathrm{p} \\
{ }^{2} \mathrm{H} \cdot{ }^{3} \mathrm{P} ;{ }^{4} \mathrm{I}\end{array}$ & 4.64 & $\begin{array}{c}3 d^{3} 4 s 4 p^{2} G \\
{ }^{3} \mathrm{P} ;{ }^{2} \mathrm{H}\end{array}$ & 0.974 & \\
\hline & 103513.555 & 103668 & 73.77 & $\begin{array}{l}3 d^{3} 4 s 4 p \\
{ }^{4} \mathrm{~F} \cdot{ }^{1} \mathrm{P} ;{ }^{4} \mathrm{G}\end{array}$ & 10.12 & $3 d^{4} 4 f^{5} D ;{ }^{4} G$ & 1.273 & \\
\hline & 105367.844 & 105330 & 79.22 & $3 d^{4} 4 f^{5} D ;{ }^{6} H$ & 10.64 & $3 d^{4} 4 f^{5} D ;{ }^{4} H$ & 1.211 & \\
\hline & 105559.523 & 105526 & 84.63 & $3 d^{4} 4 f^{5} D ;{ }^{4} H$ & 12.22 & $3 d^{4} 4 f^{5} D ;{ }^{6} H$ & 1.146 & \\
\hline & 105639.180 & 105595 & 58.42 & $3 d^{4} 4 f^{5} D ;{ }^{6} F$ & 32.94 & $3 d^{4} 4 f^{5} D ;{ }^{6} G$ & 1.396 & \\
\hline & & 105762 & 75.29 & $\begin{array}{l}3 \mathrm{~d}^{3} 4 \mathrm{~s} 4 \mathrm{p} \\
{ }^{2} \mathrm{H} \cdot{ }^{3} \mathrm{P} ;{ }^{2} \mathrm{H}\end{array}$ & 14.19 & $\begin{array}{c}3 d^{3} 4 s 4 p^{2} \mathrm{G} \\
{ }^{3} \mathrm{P} ;{ }^{2} \mathrm{H}\end{array}$ & 1.104 & \\
\hline & & 105834 & 57.74 & $3 d^{4} 4 f^{5} D ;{ }^{6} G$ & 39.32 & $3 d^{4} 4 f^{5} \mathrm{D} ;{ }^{6} \mathrm{~F}$ & 1.383 & \\
\hline & 106032.141 & 106015 & 48.97 & $3 d^{4} 4 f^{5} D ;{ }^{4} G$ & 38.24 & $\begin{array}{c}3 \mathrm{~d}^{3} 4 \mathrm{~s} 4 \mathrm{p}^{2} \mathrm{H} \\
{ }^{3} \mathrm{P} ;{ }^{4} \mathrm{G}\end{array}$ & 1.262 & \\
\hline & & 106271 & 44.46 & $\begin{array}{l}3 d^{3} 4 s 4 p \\
{ }^{2} \mathrm{H} \cdot{ }^{3} \mathrm{P} ;{ }^{4} \mathrm{G}\end{array}$ & 36.02 & $3 d^{4} 4 f^{5} D ;{ }^{4} G$ & 1.270 & \\
\hline & 107850.148 & 107629 & 98.85 & $\begin{array}{l}3 \mathrm{~d}^{3} 4 \mathrm{~s} 4 \mathrm{p} \\
{ }^{2} \mathrm{H} \cdot{ }^{3} \mathrm{P} ;{ }^{2} \mathrm{I}\end{array}$ & 0.43 & $\begin{array}{c}3 \mathrm{~d}^{3} 4 \mathrm{~s} 4 \mathrm{p}^{2} \mathrm{H} \\
{ }^{3} \mathrm{P} ;{ }^{2} \mathrm{H}\end{array}$ & 0.924 & \\
\hline & & 109142 & 55.22 & $3 d^{4} 5 p^{3} \mathrm{H} ;{ }^{4} \mathrm{I}$ & 39.76 & $3 d^{4} 5 p^{3} \mathrm{H} ;{ }^{4} \mathrm{H}$ & 1.035 & \\
\hline & & 109402 & 56.81 & $3 d^{4} 5 p^{3} \mathrm{H} ;{ }^{4} \mathrm{H}$ & 40.97 & $3 d^{4} 5 p^{3} \mathrm{H} ;{ }^{4} \mathrm{I}$ & 1.064 & \\
\hline & & 109909 & 89.48 & $3 d^{4} 5 p^{3} \mathrm{H} ;{ }^{2} \mathrm{I}$ & 5.39 & $3 d^{4} 5 p^{3} \mathrm{H} ;{ }^{2} \mathrm{H}$ & 0.939 & \\
\hline & & 110070 & 67.82 & $3 d^{4} 5 p^{3} \mathrm{H} ;{ }^{2} \mathrm{H}$ & 17.91 & $3 d^{4} 5 p^{3} \mathrm{H} ;{ }^{4} \mathrm{G}$ & 1.116 & \\
\hline & & 110279 & 99.79 & $3 d^{4} 6 p^{5} \mathrm{D} ;{ }^{6} \mathrm{~F}$ & 0.10 & $3 d^{4} 5 p^{5} D ;{ }^{6} F$ & 1.456 & \\
\hline & & 110500 & 68.83 & $3 d^{4} 5 p^{3} \mathrm{H} ;{ }^{4} \mathrm{G}$ & 20.96 & $3 d^{4} 5 p^{3} \mathrm{H} ;{ }^{2} \mathrm{H}$ & 1.232 & \\
\hline & & 111889 & 75.21 & $3 d^{4} 5 p{ }^{3} F ;{ }^{4} G$ & 21.82 & $3 d^{4} 5 p^{3} F ;{ }^{4} G$ & 1.271 & \\
\hline & & 113992 & 90.82 & $3 d^{4} 5 p^{3} \mathrm{G} ;{ }^{4} \mathrm{H}$ & 3.71 & $3 d^{4} 5 p^{3} G ;{ }^{2} \mathrm{H}$ & 1.137 & \\
\hline & & 114387 & 65.95 & $3 d^{4} 5 p^{3} G ;{ }^{4} G$ & 24.49 & $3 d^{4} 5 p^{3} G ;{ }^{2} \mathrm{H}$ & 1.218 & \\
\hline & & 114713 & 68.47 & $3 d^{4} 5 p^{3} \mathrm{G} ;{ }^{2} \mathrm{H}$ & 27.18 & $3 d^{4} 5 p^{3} G ;{ }^{4} G$ & 1.144 & \\
\hline & 115396.773 & 115409 & 65.73 & $3 d^{4} 5 f^{5} D ;{ }^{6} \mathrm{H}$ & 17.89 & $3 d^{4} 4 f^{5} D ;{ }^{6} G$ & 1.231 & \\
\hline & 115585.117 & 115590 & 28.82 & $3 d^{4} 5 f^{5} D ;{ }^{6} H$ & 26.38 & $3 d^{3} 4 s 4 p^{5} \mathrm{D} ;{ }^{4} \mathrm{H}$ & 1.269 & \\
\hline & 115598.883 & 115604 & 54.03 & $3 d^{4} 5 f^{5} D ;{ }^{4} H$ & 26.72 & $3 d^{4} 4 p^{5} \mathrm{D} ;{ }^{6} \mathrm{~F}$ & 1.259 & \\
\hline & 115840.391 & 115826 & 53.66 & $3 d^{4} 5 f^{5} D ;{ }^{6} G$ & 41.58 & $3 d^{4} 4 p^{5} D ;{ }^{6} F$ & 1.383 & \\
\hline & 115916.148 & 115925 & 87.43 & $3 d^{4} 5 f^{5} D ;{ }^{4} G$ & 7.93 & $3 d^{3} 4 s 4 p^{5} \mathrm{D} ;{ }^{4} \mathrm{H}$ & 1.266 & \\
\hline & & 116516 & 59.53 & $3 d^{4} 5 p^{1} \mathrm{I} ;{ }^{2} \mathrm{H}$ & 29.10 & $\begin{array}{l}3 \mathrm{~d}^{3} 4 \mathrm{~s} 4 \mathrm{p}{ }^{2} \mathrm{G} \\
{ }^{1} \mathrm{P} ;{ }^{2} \mathrm{H}\end{array}$ & 1.087 & \\
\hline & & 116889 & 94.76 & $\begin{array}{l}3 d^{3} 4 s 4 p \\
{ }^{2} \mathrm{~F}^{3} \mathrm{P} ;{ }^{4} \mathrm{G}\end{array}$ & 3.63 & $\begin{array}{l}3 \mathrm{~d}^{3} 4 \mathrm{~s} 4 \mathrm{p}^{2} \mathrm{H} \\
{ }^{3} \mathrm{P} ;{ }^{4} \mathrm{G}\end{array}$ & 1.273 & \\
\hline & & 117240 & 93.51 & $3 d^{4} 5 p^{1} \mathrm{I} ;{ }^{2} \mathrm{I}$ & 2.43 & $3 \mathrm{~d}^{3} 4 \mathrm{~s} 4 \mathrm{p}^{2} \mathrm{H} .{ }^{1} \mathrm{P} ;{ }^{2} \mathrm{I}$ & 0.928 & \\
\hline & & 118451 & 56.37 & $3 d^{4} 5 p{ }^{1} G ;{ }^{2} \mathrm{H}$ & 30.71 & $3 d^{4} 5 p{ }^{1} G ;{ }^{2} \mathrm{H}$ & 1.091 & \\
\hline & & 119621 & 49.41 & $\begin{array}{l}3 \mathrm{~d}^{3} 4 \mathrm{~s} 4 \mathrm{p} \\
{ }^{2} \mathrm{G} \cdot{ }^{1} \mathrm{P} ;{ }^{2} \mathrm{H}\end{array}$ & 26.97 & $3 d^{4} 5 p{ }^{1} \mathrm{I} ;{ }^{2} \mathrm{H}$ & 1.089 & \\
\hline & & 120664 & 56.15 & $3 d^{4} 4 f^{3} \mathrm{H} ;{ }^{2} \mathrm{I}$ & 23.09 & $3 \mathrm{~d}^{4} 4 \mathrm{f}^{3} \mathrm{H} ;{ }^{2} \mathrm{H}$ & 0.968 & \\
\hline & & 120727 & 79.31 & $3 \mathrm{~d}^{4} 4 \mathrm{f}^{3} \mathrm{H} ;{ }^{4} \mathrm{~K}$ & 15.79 & $3 \mathrm{~d}^{4} 4 \mathrm{f}^{3} \mathrm{H} ;{ }^{4} \mathrm{I}$ & 0.813 & \\
\hline & & 120793 & 39.81 & $3 \mathrm{~d}^{4} 4 \mathrm{f}^{3} \mathrm{H} ;{ }^{4} \mathrm{I}$ & 36.30 & $3 \mathrm{~d}^{4} 4 \mathrm{f}^{3} \mathrm{H} ;{ }^{2} \mathrm{H}$ & 1.011 & \\
\hline & & 120857 & 28.30 & $3 \mathrm{~d}^{4} 4 \mathrm{f}^{3} \mathrm{H} ;{ }^{2} \mathrm{I}$ & 23.95 & $3 \mathrm{~d}^{4} 4 \mathrm{f}^{3} \mathrm{H} ;{ }^{4} \mathrm{I}$ & 1.006 & \\
\hline & & 120951 & 60.81 & $3 \mathrm{~d}^{4} 4 \mathrm{f}^{3} \mathrm{H} ;{ }^{4} \mathrm{H}$ & 17.40 & $3 d^{4} 4 f^{3} \mathrm{H} ;{ }^{4} \mathrm{I}$ & 1.103 & \\
\hline \multicolumn{9}{|l|}{6.5} \\
\hline & 64030.504 & 64035 & 84.09 & $3 d^{4} 4 p^{3} \mathrm{H} ;{ }^{4} \mathrm{H}$ & 13.90 & $3 d^{4} 4 p^{3} G ;{ }^{4} \mathrm{H}$ & 1.229 & \\
\hline & 65617.945 & 65546 & 98.19 & $3 d^{4} 4 p^{3} \mathrm{H} ;{ }^{4} \mathrm{I}$ & 1.47 & $3 \mathrm{~d}^{4} 4 \mathrm{p}^{3} \mathrm{H} ;{ }^{4} \mathrm{H}$ & 1.110 & \\
\hline & 67588.875 & 67773 & 92.21 & $3 d^{4} 4 p^{3} \mathrm{H} ;{ }^{2} \mathrm{I}$ & 5.69 & $3 d^{4} 4 \mathrm{p}^{1} \mathrm{I} ;{ }^{2} \mathrm{I}$ & 1.079 & \\
\hline & 69388.148 & 69436 & 84.00 & $3 d^{4} 4 p^{3} G ;{ }^{4} \mathrm{H}$ & 13.97 & $3 d^{4} 4 p^{3} \mathrm{H} ;{ }^{4} \mathrm{H}$ & 1.229 & \\
\hline
\end{tabular}


Table 5 (continued)

\begin{tabular}{|c|c|c|c|c|c|c|c|c|}
\hline \multirow[t]{25}{*}{$J$-value } & \multirow{2}{*}{$\begin{array}{c}E_{\text {obs }} \\
\left(\mathrm{cm}^{-1}\right) \\
{[8,27]} \\
74424.156\end{array}$} & \multirow{2}{*}{$\begin{array}{c}\begin{array}{c}E_{\text {calc }} \\
\left(\mathrm{cm}^{-1}\right)\end{array} \\
74291\end{array}$} & \multicolumn{2}{|c|}{$\begin{array}{l}\text { Largest eigenvalue component } \\
(\%)\end{array}$} & \multicolumn{2}{|c|}{$\begin{array}{l}\text { Next largest eigenvalue component } \\
(\%)\end{array}$} & \multirow{2}{*}{$\begin{array}{c}\text { Calc. } g_{J} \\
0.962\end{array}$} & \multirow{2}{*}{$\begin{array}{c}\text { Obs. } g_{J} \\
{[5]}\end{array}$} \\
\hline & & & 79.99 & $3 \mathrm{~d}^{4} 4 \mathrm{p}^{1} \mathrm{I} ;{ }^{2} \mathrm{~K}$ & 19.28 & $3 d^{4} 4 \mathrm{p}^{1} \mathrm{I} ;{ }^{2} \mathrm{I}$ & & \\
\hline & 74743.180 & 74613 & 74.62 & $3 d^{4} 4 \mathrm{p}^{1} \mathrm{I} ;{ }^{2} \mathrm{I}$ & 19.73 & $3 \mathrm{~d}^{4} 4 \mathrm{p}^{1} \mathrm{I} ;{ }^{2} \mathrm{~K}$ & 1.049 & \\
\hline & & 80313 & 99.89 & $\begin{array}{l}3 d^{3} 4 s 4 p \\
{ }^{4} \mathrm{~F} \cdot{ }^{3} \mathrm{P} ;{ }^{6} \mathrm{G}\end{array}$ & 0.06 & $\begin{array}{c}3 \mathrm{~d}^{3} 4 \mathrm{~s} 4 \mathrm{p}^{2} \mathrm{G} \\
{ }^{3} \mathrm{P} ;{ }^{4} \mathrm{H}\end{array}$ & 1.385 & \\
\hline & & 97013 & 83.57 & $\begin{array}{l}3 d^{3} 4 s 4 p \\
{ }^{2} \mathrm{G} \cdot{ }^{3} \mathrm{P} ;{ }^{4} \mathrm{H}\end{array}$ & 15.78 & $\begin{array}{c}3 d^{3} 4 s 4 p^{2} \mathrm{H} \\
{ }^{3} \mathrm{P} ;{ }^{4} \mathrm{H}\end{array}$ & 1.231 & \\
\hline & 101900.578 & 101843 & 83.30 & $\begin{array}{l}3 \mathrm{~d}^{3} 4 \mathrm{~s} 4 \mathrm{p} \\
{ }^{2} \mathrm{H} \cdot{ }^{3} \mathrm{P} ;{ }^{4} \mathrm{H}\end{array}$ & 15.67 & $\begin{array}{c}3 \mathrm{~d}^{3} 4 \mathrm{~s} 4 \mathrm{p}^{2} \mathrm{G} \\
{ }^{3} \mathrm{P} ;{ }^{4} \mathrm{H}\end{array}$ & 1.231 & \\
\hline & & 103317 & 99.50 & $\begin{array}{l}3 \mathrm{~d}^{3} 4 \mathrm{~s} 4 \mathrm{p} \\
{ }^{2} \mathrm{H} \cdot{ }^{3} \mathrm{P} ;{ }^{4} \mathrm{I}\end{array}$ & 0.20 & $\begin{array}{c}3 d^{3} 4 s 4 p^{2} G \\
{ }^{3} \mathrm{P} ;{ }^{4} \mathrm{H}\end{array}$ & 1.108 & \\
\hline & & 105455 & 87.40 & $3 d^{4} 4 f^{5} \mathrm{D} ;{ }^{6} \mathrm{H}$ & 6.50 & $3 d^{4} 4 f^{5} D ;{ }^{6} G$ & 1.286 & \\
\hline & 105742.453 & 105691 & 91.40 & $3 d^{4} 4 f^{5} D ;{ }^{4} H$ & 7.65 & $3 d^{4} 4 f^{5} D ;{ }^{6} H$ & 1.236 & \\
\hline & 105895.461 & 105832 & 92.58 & $3 d^{4} 4 f^{5} D ;{ }^{6} G$ & 4.91 & $3 d^{4} 4 f^{5} D ;{ }^{6} H$ & 1.377 & \\
\hline & & 107785 & 99.12 & $\begin{array}{l}3 \mathrm{~d}^{3} 4 \mathrm{~s} 4 \mathrm{p} \\
{ }^{2} \mathrm{H} \cdot{ }^{3} \mathrm{P} ;{ }^{2} \mathrm{I}\end{array}$ & 0.25 & $3 d^{4} 5 p^{1} \mathrm{I} ;{ }^{2} \mathrm{I}$ & 1.077 & \\
\hline & & 109289 & 55.45 & $3 d^{4} 5 p^{3} \mathrm{H} ;{ }^{4} \mathrm{H}$ & 37.27 & $3 d^{4} 5 p^{3} \mathrm{H} ;{ }^{4} \mathrm{I}$ & 1.176 & \\
\hline & & 109554 & 61.14 & $3 d^{4} 5 p^{3} \mathrm{H} ;{ }^{4} \mathrm{I}$ & 37.80 & $3 d^{4} 5 p^{3} \mathrm{H} ;{ }^{4} \mathrm{H}$ & 1.155 & \\
\hline & & 110023 & 92.75 & $3 d^{4} 5 p^{3} \mathrm{H} ;{ }^{2} \mathrm{I}$ & 5.24 & $3 d^{4} 5 p^{3} \mathrm{H} ;{ }^{4} \mathrm{H}$ & 1.086 & \\
\hline & & 114228 & 98.81 & $3 d^{4} 5 p^{3} G ;{ }^{4} H$ & 0.46 & $3 d^{4} 5 p^{3} \mathrm{H} ;{ }^{4} \mathrm{H}$ & 1.231 & \\
\hline & 115546.523 & 115543 & 80.16 & $3 d^{4} 5 f^{5} D ;{ }^{6} H$ & 13.05 & $3 d^{4} 5 f^{5} D ;{ }^{6} G$ & 1.293 & \\
\hline & 115782.797 & 115774 & 82.52 & $3 d^{4} 5 f^{5} D ;{ }^{4} H$ & 12.47 & $3 d^{4} 5 f^{5} D ;{ }^{6} H$ & 1.245 & \\
\hline & 115836.531 & 115822 & 81.99 & $3 d^{4} 5 f^{5} D ;{ }^{6} G$ & 10.60 & $3 d^{4} 5 f^{5} D ;{ }^{4} H$ & 1.362 & \\
\hline & & 117121 & 53.81 & $3 d^{4} 5 p^{1} \mathrm{I} ;{ }^{2} \mathrm{I}$ & 44.33 & $3 \mathrm{~d}^{4} 5 \mathrm{p}^{1} \mathrm{I} ;{ }^{2} \mathrm{~K}$ & 1.013 & \\
\hline & & 117468 & 55.38 & $3 d^{4} 5 p^{1} \mathrm{I} ;{ }^{2} \mathrm{~K}$ & 43.16 & $3 d^{4} 5 p^{1}{ }^{1} ;{ }^{2} \mathrm{I}$ & 0.997 & \\
\hline & & 120759 & 59.95 & $3 \mathrm{~d}^{4} 4 \mathrm{f}^{3} \mathrm{H} ;{ }^{4} \mathrm{~K}$ & 21.11 & $3 d^{4} 4 f^{3} H ;{ }^{2} K$ & 0.982 & \\
\hline & & 120811 & 60.58 & $3 \mathrm{~d}^{4} 4 \mathrm{f}^{3} \mathrm{H} ;{ }^{4} \mathrm{~L}$ & 16.27 & $3 \mathrm{~d}^{4} 4 \mathrm{f}^{3} \mathrm{H} ;{ }^{2} \mathrm{I}$ & 0.890 & \\
\hline & & 120835 & 34.32 & $3 d^{4} 4 f^{3} \mathrm{H} ;{ }^{2} \mathrm{I}$ & 31.32 & $3 \mathrm{~d}^{4} 4 \mathrm{f}^{3} \mathrm{H} ;{ }^{4} \mathrm{~L}$ & 0.984 & \\
\hline & & 120895 & 51.96 & $3 \mathrm{~d}^{4} 4 \mathrm{f}^{3} \mathrm{H} ;{ }^{2} \mathrm{~K}$ & 32.33 & $3 d^{4} 4 f^{3} H ;{ }^{4} \mathrm{I}$ & 0.997 & \\
\hline & & 120957 & 38.03 & $3 d^{4} 4 f^{3} \mathrm{H} ;{ }^{2} \mathrm{I}$ & 27.49 & $3 d^{4} 4 f^{3} \mathrm{H} ;{ }^{4} \mathrm{I}$ & 1.044 & \\
\hline \multicolumn{9}{|c|}{ 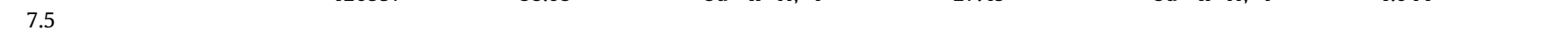 } \\
\hline & 65812.648 & 65731 & 99.72 & $3 d^{4} 4 p^{3} \mathrm{H} ;{ }^{4} \mathrm{I}$ & 0.14 & $3 d^{4} 5 p^{3} \mathrm{H} ;{ }^{4} \mathrm{I}$ & 1.200 & \\
\hline & 74958.859 & 74838 & 99.80 & $3 d^{4} 4 \mathrm{p}^{1} \mathrm{I} ;{ }^{2} \mathrm{~K}$ & 0.09 & $3 d^{4} 5 p^{1} I ;{ }^{2} K$ & 1.067 & \\
\hline & & 103567 & 99.75 & $\begin{array}{l}3 \mathrm{~d}^{3} 4 \mathrm{~s} 4 \mathrm{p} \\
{ }^{2} \mathrm{H} \cdot{ }^{3} \mathrm{P} ;{ }^{4} \mathrm{I}\end{array}$ & 0.16 & $3 d^{4} 5 p^{3} \mathrm{H} ;{ }^{4} \mathrm{I}$ & 1.200 & \\
\hline & & 105614 & 99.95 & $3 d^{4} 4 f^{5} D ;{ }^{6} H$ & 0.03 & $3 \mathrm{~d}^{4} 4 \mathrm{f}^{3} \mathrm{~F} ;{ }^{4} \mathrm{I}$ & 1.334 & \\
\hline & & 109668 & 99.53 & $3 d^{4} 5 p^{3} \mathrm{H} ;{ }^{4} \mathrm{I}$ & 0.16 & $3 \mathrm{~d}^{3} 4 \mathrm{~s} 4 \mathrm{p}^{2} \mathrm{H} \cdot{ }^{3} \mathrm{P} ;{ }^{4} \mathrm{I}$ & 1.200 & \\
\hline & 115734.766 & 115715 & 99.95 & $3 \mathrm{~d}^{4} 5 \mathrm{f}^{5} \mathrm{D} ;{ }^{6} \mathrm{H}$ & 0.03 & $3 d^{4} 5 f^{3} F ;{ }^{4} I$ & 1.334 & \\
\hline & & 117607 & 99.73 & $3 \mathrm{~d}^{4} 5 \mathrm{p}^{1} \mathrm{I} ;{ }^{2} \mathrm{~K}$ & 0.11 & $3 d^{4} 5 p^{3} \mathrm{H} ;{ }^{4} \mathrm{I}$ & 1.067 & \\
\hline & & 120819 & 36.33 & $3 d^{4} 4 f^{3} H ;{ }^{2} L$ & 31.20 & $3 \mathrm{~d}^{4} 4 \mathrm{f}^{3} \mathrm{H} ;{ }^{4} \mathrm{~L}$ & 0.998 & \\
\hline & & 120867 & 53.55 & $3 \mathrm{~d}^{4} 4 \mathrm{f}^{3} \mathrm{H} ;{ }^{4} \mathrm{~K}$ & 18.92 & $3 d^{4} 4 f^{3} H ;{ }^{2} \mathrm{~L}$ & 1.053 & \\
\hline & & 120941 & 51.51 & $3 d^{4} 4 f^{3} H ;{ }^{4} \mathrm{~L}$ & 40.91 & $3 d^{4} 4 f^{3} H ;{ }^{2} L$ & 0.964 & \\
\hline & & 120986 & 86.16 & $3 \mathrm{~d}^{4} 4 \mathrm{f}^{3} \mathrm{H} ;{ }^{4} \mathrm{I}$ & 11.87 & $3 \mathrm{~d}^{4} 4 \mathrm{f}^{3} \mathrm{H} ;{ }^{4} \mathrm{~K}$ & 1.183 & \\
\hline \multicolumn{9}{|l|}{8.5} \\
\hline & & 120930 & 54.30 & $3 d^{4} 4 f^{3} H ;{ }^{4} L$ & 27.93 & $3 \mathrm{~d}^{4} 4 \mathrm{f}^{3} \mathrm{H} ;{ }^{4} \mathrm{~K}$ & 1.102 & \\
\hline & & 120982 & 71.89 & $3 \mathrm{~d}^{4} 4 \mathrm{f}^{3} \mathrm{H} ;{ }^{4} \mathrm{~K}$ & 21.72 & $3 d^{4} 4 f^{3} H ;{ }^{4} \mathrm{~L}$ & 1.148 & \\
\hline & & 121076 & 76.13 & $3 \mathrm{~d}^{4} 4 \mathrm{f}^{3} \mathrm{H} ;{ }^{2} \mathrm{~L}$ & 23.73 & $3 \mathrm{~d}^{4} 4 \mathrm{f}^{3} \mathrm{H} ;{ }^{4} \mathrm{~L}$ & 1.063 & \\
\hline \multicolumn{9}{|c|}{ 然 } \\
\hline & & 121062 & 99.89 & $3 d^{4} 4 f^{3} H ;{ }^{4} L$ & 0.11 & $3 d^{4} 4 f^{1} \mathrm{I} ;{ }^{2} \mathrm{M}$ & 1.158 & \\
\hline & & 128722 & 99.89 & $3 \mathrm{~d}^{4} 4 \mathrm{f}^{1} \mathrm{I} ;{ }^{2} \mathrm{M}$ & 0.11 & $3 \mathrm{~d}^{4} 4 \mathrm{f}^{3} \mathrm{H} ;{ }^{4} \mathrm{~L}$ & 1.053 & \\
\hline & & 131091 & 99.89 & $3 \mathrm{~d}^{4} 5 \mathrm{f}^{3} \mathrm{H} ;{ }^{4} \mathrm{~L}$ & 0.11 & $3 \mathrm{~d}^{4} 5 \mathrm{f}^{1} \mathrm{I} ;{ }^{2} \mathrm{M}$ & 1.158 & \\
\hline
\end{tabular}


Table 6

Values of the intra-configuration fine structure parameters for $\mathrm{Cr}$ II odd configurations.

\begin{tabular}{|c|c|c|}
\hline Configuration & Parameter & $\begin{array}{l}\text { Value } \\
\left(\mathrm{cm}^{-1}\right)\end{array}$ \\
\hline $3 d^{4} 4 p$ & $\begin{array}{l}E_{a v} \\
\mathrm{~F}^{2}(3 \mathrm{~d}, 3 \mathrm{~d}) \\
\mathrm{F}^{4}(3 \mathrm{~d}, 3 \mathrm{~d}) \\
\mathrm{F}^{2}(3 \mathrm{~d}, 4 \mathrm{p}) \\
\mathrm{G}^{1}(3 \mathrm{~d}, 4 \mathrm{p}) \\
\mathrm{G}^{3}(3 \mathrm{~d}, 4 \mathrm{p}) \\
\zeta_{3 d} \\
\zeta_{4 p} \\
\alpha \\
\beta \\
\mathrm{T}_{1} \\
\mathrm{~T}_{2}\end{array}$ & $\begin{array}{r}74271(10) \\
60474(54) \\
35556(83) \\
10838(88) \\
5081(38) \\
1023(74) \\
218(13) \\
254(19) \\
54(1) \\
-140(14) \\
42(25) \\
-237(38)\end{array}$ \\
\hline $3 d^{4} 5 p$ & $\begin{array}{l}E_{a v} \\
\mathrm{~F}^{2}(3 \mathrm{~d}, 3 \mathrm{~d}) \\
\mathrm{F}^{4}(3 \mathrm{~d}, 3 \mathrm{~d}) \\
\mathrm{F}^{2}(3 \mathrm{~d}, 5 \mathrm{p}) \\
\mathrm{G}^{1}(3 \mathrm{~d}, 5 \mathrm{p}) \\
\mathrm{G}^{3}(3 \mathrm{~d}, 5 \mathrm{p}) \\
\zeta_{3 d} \\
\zeta_{5 p} \\
\alpha \\
\beta \\
\mathrm{T}_{1} \\
\mathrm{~T}_{2}\end{array}$ & $\begin{array}{r}11874(30) \\
65945(530) \\
37859(518) \\
2619^{d} \\
1173^{d} \\
9406^{d} \\
218^{d} \\
81^{d} \\
0^{f} \\
0^{f} \\
0^{f} \\
0^{f}\end{array}$ \\
\hline $3 d^{4} 6 p$ & $\begin{array}{l}E_{a v} \\
\mathrm{~F}^{2}(3 \mathrm{~d}, 3 \mathrm{~d}) \\
\mathrm{F}^{4}(3 \mathrm{~d}, 3 \mathrm{~d}) \\
\mathrm{F}^{2}(3 \mathrm{~d}, 6 \mathrm{p}) \\
\mathrm{G}^{1}(3 \mathrm{~d}, 6 \mathrm{p}) \\
\mathrm{G}^{3}(3 \mathrm{~d}, 6 \mathrm{p}) \\
\zeta_{3 d} \\
\zeta_{6 p} \\
\alpha \\
\beta \\
\mathrm{T}_{1} \\
\mathrm{~T}_{2}\end{array}$ & $\begin{array}{r}134515(39) \\
64111(650) \\
38164(635) \\
1148^{d} \\
647^{d} \\
400^{d} \\
218^{d} \\
36^{d} \\
0^{f} \\
0^{f} \\
0^{f} \\
0^{f}\end{array}$ \\
\hline $3 d^{3} 4 s 4 p$ & $\begin{array}{l}E_{a v} \\
\mathrm{~F}^{2}(3 \mathrm{~d}, 3 \mathrm{~d}) \\
\mathrm{F}^{4}(3 \mathrm{~d}, 3 \mathrm{~d}) \\
\mathrm{F}^{2}(3 \mathrm{~d}, 4 \mathrm{p}) \\
\mathrm{G}^{1}(4 \mathrm{~s}, 4 \mathrm{p}) \\
\mathrm{G}^{2}(3 \mathrm{~d}, 4 \mathrm{~s}) \\
\mathrm{G}^{1}(3 \mathrm{~d}, 4 \mathrm{p}) \\
\mathrm{G}^{3}(3 \mathrm{~d}, 4 \mathrm{p}) \\
\zeta_{3 d} \\
\zeta_{4 p} \\
\alpha \\
\beta \\
\mathrm{T}_{s} \\
\mathrm{~T}_{1} \\
\mathrm{~T}_{2}\end{array}$ & $\begin{array}{r}107441(14) \\
67824(127) \\
40200(146) \\
14457(177) \\
27810(105) \\
6507(191) \\
6166(110) \\
1576(287) \\
293(17) \\
382(57) \\
53(1) \\
-180(27) \\
174(33) \\
199(43) \\
-207(41)\end{array}$ \\
\hline $3 d^{4} 4 f$ & $\begin{array}{l}E_{a v} \\
\mathrm{~F}^{2}(3 \mathrm{~d}, 3 \mathrm{~d}) \\
\mathrm{F}^{4}(3 \mathrm{~d}, 3 \mathrm{~d}) \\
\mathrm{F}^{2}(3 \mathrm{~d}, 4 \mathrm{f}) \\
\mathrm{F}^{4}(3 \mathrm{~d}, 4 \mathrm{f}) \\
\mathrm{G}^{1}(3 \mathrm{~d}, 4 \mathrm{f}) \\
\mathrm{G}^{3}(3 \mathrm{~d}, 4 \mathrm{f}) \\
\mathrm{G}^{5}(3 \mathrm{~d}, 4 \mathrm{f}) \\
\zeta_{3 d} \\
\zeta_{4 f} \\
\alpha \\
\beta \\
\mathrm{T}_{1} \\
\mathrm{~T}_{2}\end{array}$ & $\begin{array}{r}129606(27) \\
63513^{d} \\
37545^{d} \\
1000^{d} \\
200^{d} \\
130^{d} \\
77^{d} \\
0^{f} \\
220^{d} \\
0^{f} \\
0^{f} \\
0^{f} \\
0^{f} \\
0^{f}\end{array}$ \\
\hline
\end{tabular}


Table 6 (continued)

\begin{tabular}{|c|c|c|}
\hline Configuration & Parameter & $\begin{array}{l}\text { Value } \\
\left(\mathrm{cm}^{-1}\right)\end{array}$ \\
\hline $3 d^{4} 5 f$ & $\begin{array}{l}E_{a v} \\
\mathrm{~F}^{2}(3 \mathrm{~d}, 3 \mathrm{~d}) \\
\mathrm{F}^{4}(3 \mathrm{~d}, 3 \mathrm{~d}) \\
\mathrm{F}^{2}(3 \mathrm{~d}, 5 \mathrm{f}) \\
\mathrm{F}^{4}(3 \mathrm{~d}, 5 \mathrm{f}) \\
\mathrm{G}^{1}(3 \mathrm{~d}, 5 \mathrm{f}) \\
\mathrm{G}^{3}(3 \mathrm{~d}, 5 \mathrm{f}) \\
\mathrm{G}^{5}(3 \mathrm{~d}, 5 \mathrm{f}) \\
\zeta_{3 d} \\
\zeta_{5 f} \\
\alpha \\
\beta \\
\mathrm{T}_{1} \\
\mathrm{~T}_{2}\end{array}$ & $\begin{array}{r}139660(23) \\
63492^{d} \\
37554^{d} \\
510^{d} \\
120^{d} \\
100^{d} \\
55^{d} \\
0^{f} \\
220^{d} \\
0^{f} \\
0^{f} \\
0^{f} \\
0^{f} \\
0^{f}\end{array}$ \\
\hline
\end{tabular}

${ }^{f}$ Frozen to zero.

${ }^{d}$ Deduced from $a b$ initio or fs calculations. 
Table 7

Values of odd configuration interaction parameters.

\begin{tabular}{|c|c|c|}
\hline Configurations & Parameter $^{a}$ & $\begin{array}{l}\text { Value } \\
\left(\mathrm{cm}^{-1}\right)\end{array}$ \\
\hline $3 d^{4} 4 p-3 d^{3} 4 s 4 p$ & $\begin{array}{l}\mathrm{R}^{2} \\
\mathrm{D}^{2} \\
\mathrm{E}^{1}\end{array}$ & $\begin{array}{r}-5507(215) \\
-4813(253) \\
-4813^{d}\end{array}$ \\
\hline $3 d^{4} 4 p-3 d^{4} 5 p$ & $\begin{array}{l}E^{1} \\
E^{3}\end{array}$ & $\begin{array}{l}2389(224) \\
1620(530)\end{array}$ \\
\hline $3 d^{3} 4 s 4 p-3 d^{4} 5 p$ & $\begin{array}{l}D^{2} \\
E^{1}\end{array}$ & $\begin{array}{l}-3225(278) \\
-3443(165)\end{array}$ \\
\hline
\end{tabular}

${ }^{a}$ For meaning of $\mathrm{R}^{2}, \mathrm{D}^{2}, \mathrm{E}^{1}$ and $\mathrm{E}^{3}$ see Cowan's book [28].

${ }^{d}$ Deduced from $a b$ initio or fs calculations. 